LA WRENCE LIWEAMCAE WATIONAL LABOAATOAY

\title{
FY2003 Annual Report: DOE Project on Heavy Vehicle Aerodynamic Drag
}

R.C. McCallen, K. Salari, J. Ortega, LLNL; L.J. DeChant, C.J. Roy, J.L. Payne, B. Hassan, SNL; W.D. Pointer, ANL; F. Browand, M. Hammache, T.-Y. Hsu, USC; J. Ross, D. Satran, J.T. Heineck, S. Walker, D. Yaste, NASA Ames; R. Englar, GTRI; A. Leonard, M. Rubel, P. Chatelain, Caltech

November 1, 2003 
This document was prepared as an account of work sponsored by an agency of the United States Government. Neither the United States Government nor the University of California nor any of their employees, makes any warranty, express or implied, or assumes any legal liability or responsibility for the accuracy, completeness, or usefulness of any information, apparatus, product, or process disclosed, or represents that its use would not infringe privately owned rights. Reference herein to any specific commercial product, process, or service by trade name, trademark, manufacturer, or otherwise, does not necessarily constitute or imply its endorsement, recommendation, or favoring by the United States Government or the University of California. The views and opinions of authors expressed herein do not necessarily state or reflect those of the United States Government or the University of California, and shall not be used for advertising or product endorsement purposes.

This work was performed under the auspices of the U.S. Department of Energy by University of California, Lawrence Livermore National Laboratory under Contract W-7405-Eng-48. 


\section{DOE Project on Heavy Vehicle Aerodynamic Drag}

Project Principal Investigator: $R$. C. McCallen

Lawrence Livermore National Laboratory

P.O. Box 808, Livermore, CA 94551-0808

(925)423-0958; mccallen1@llnl.gov

Principal Investigator: K. Salari

Co-Investigators: J. Ortega

Lawrence Livermore National Laboratory

P.O. Box 808, Livermore, CA 94551-0808

(925) 424-4635; salari1@llnl.gov

Principal Investigators: L. J. DeChant

Co-Investigators: C. J. Roy, J. L. Payne, B. Hassan

Sandia National Laboratories

P.O. Box 5800, MS 0825, Albuquerque, NM 87185-0825

(505)844-4250ljdecha@sandia.gov

Principal Investigator: W. D. Pointer

Argonne National Laboratory

9700 S. Cass Avenue, NE-208, Argonne, IL 60439

(630) 252-1052;dpointer@anl.gov

Principal Investigator: F. Browand

Co-Investigators: M. Hammache, T.-Y. Hsu

Aerospace \& Mechanical Engineering, University of Southern California

RRB 203, Los Angeles CA 90089-1191

(213) 740-5359; e-mail: browand@spock.usc.edu

Principal Investigator: J. Ross

Co-Investigators: D. Satran, J.T. Heineck, S. Walker, D. Yaste

NASA Ames Research Center

MS 260-1, Moffett Field, CA 94035

(650)604-6722; jcross@mail.arc.nasa.gov

Principal Investigator: $R$. Englar

Georgia Tech Research Institute

ATASL, CCRF, Atlanta, GA 30332-0844

(770) 528-3222; bob.englar@gtri.gatech.edu 
Principal Investigator: A. Leonard

Co-Investigator: M. Rubel, P. Chatelain

California Institute of Technology

1200 East California Blvd. MC 301-46, Pasadena, CA 91125

(626) 395-4465; tony@galcit.caltech.edu

Technology Development Manager: Sid Diamond

202-586-8032, sid.diamond@ee.doe.gov

Technical Program Manager: Jules Routbort

630-252-5065,routbort@anl.gov

Contractor: Lawrence Livermore National Laboratory, Sandia National Laboratories, Argonne National Laboratory, NASA Ames Research Center, Georgia Tech Research Institute, University of Southern California, California Institute of California

Contract No.: W-7405-ENG-48, DE-AC04-94AL85000, W-31-109-ENG-38, DE-AI01-99EE50559, DE-AC03-02EE5069, DE-AC03-98EE50512, DE-ACO3$98 E E 50506$

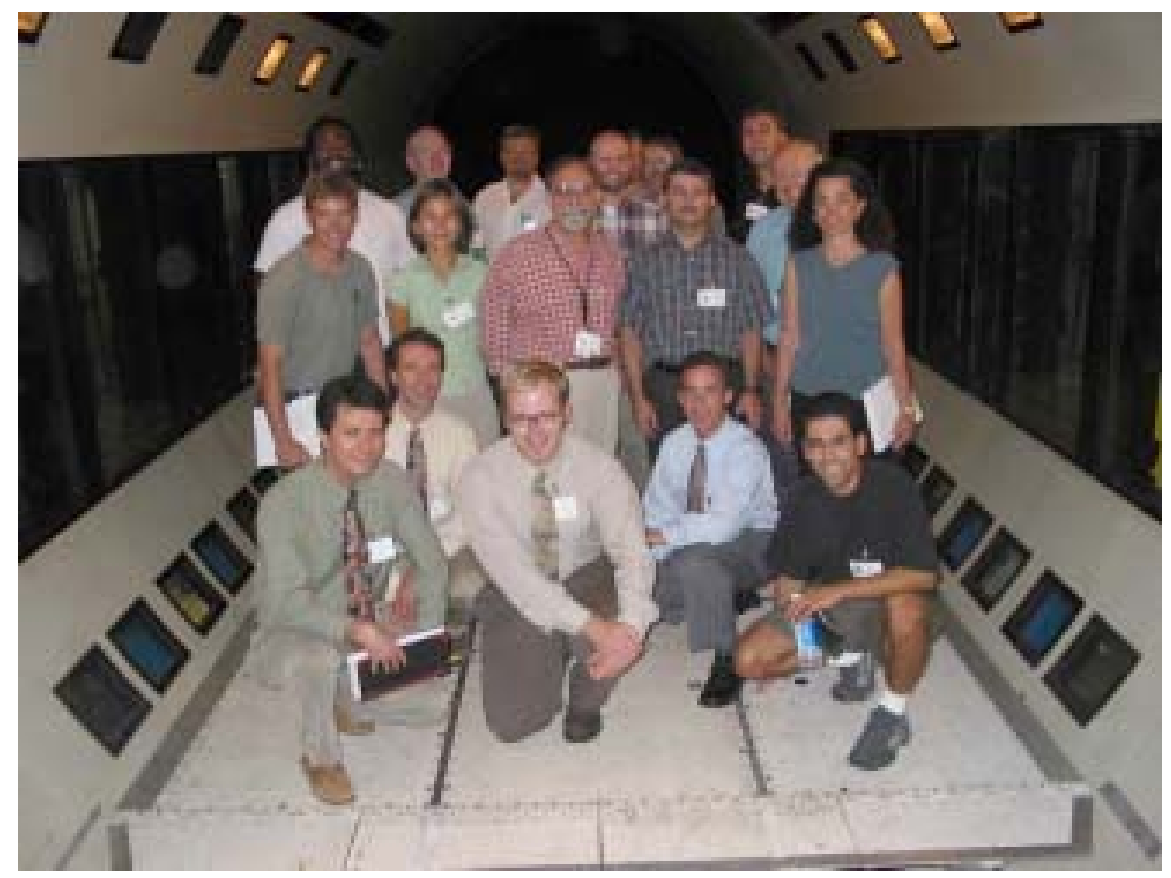

Consortium members in NASA Ames Research Center 12-foot pressure wind tunnel. 


\section{Objective}

- Provide guidance to industry in the reduction of aerodynamic drag of heavy truck vehicles.

- Establish a database of experimental, computational, and conceptual design information, and demonstrate potential of new drag-reduction devices.

\section{Approach}

- Develop and demonstrate the ability to simulate and analyze aerodynamic flow around heavy truck vehicles using existing and advanced computational fluid dynamics (CFD) tools.

- Through an extensive experimental effort, generate an experimental data base for code validation.

- Using experimental data base, validate computations.

- Provide industry with design guidance and insight into flow phenomena from experiments and computations.

- Investigate aero devices (e.g., boattail plates, side extenders, blowing and acoustic devices) and provide industry with conceptual designs of drag reducing devices. Demonstrate the full-scale fuel economy potential of these devices.

\section{Accomplishments}

- Drag reduction devices met project goals:

○ Angled plates provide 50\% more drag reduction than boattail plates

○ Use of angled plates $(\sim 15 \%)$ with skirts or lowboy trailers $(\sim 10 \%)$ provides over $20 \%$ reduction in drag which corresponds to more than $10 \%$ reduction in fuel use at highway speeds.

- Track tests of the pneumatic blowing device demonstrated a maximum (best case) reduction of 5-6\% in fuel use (not accounting for fuel use for blowing). Recent wind-tunnel tests have identified configuration/blowing improvements indicating potential fuel economy of $7 \%$ from drag reduction of $14 \%$ due to blowing alone.

- Full-scale test results on an SUV indicate the potential for blowing to reduce drag (increase fuel economy), increase drag for braking, reduce drag due to side winds, and provide directional stability, without moving parts.

○ Computationally showed that use of a "splitter plate" in the tractortrailer gap may be one way to maintain a symmetrical, low drag condition.

- Insight from experiments and experimental data base provided clear guidance to industry on reliable, predictable experimental techniques:

- Reynolds number $(\mathrm{Re})$ effects on the vehicle drag coefficient $\left(\mathrm{C}_{\mathrm{D}}\right)$ are in general minimal for experiments with Re above 1 million. 
This finding supports the common use of scaled down vehicles and Re below typical highway Re for experimentation.

$\circ$ Experiments indicate that inaccuracies should be considered when evaluating gap and wake drag reduction devices at lower than highway Re. Low Re experiments should provide ball park estimates, but accurate optimization of devices may require road testing.

- Edge radius effects and/or the cleanliness of the vehicle upstream flow are critical to achieving accurate predictions.

- Drag measurements alone are not sufficient to provide an understanding of the impact of geometry modifications and direction for design improvements and advanced measurement techniques (e.g., particle-image velocimetry, unsteady pressure taps, oil film interferometry) should be included to provide important information on the global and local structure of the flow and clear design direction.

- Computational results provided the following clear guidance and caution warnings on the use of steady Reynolds-averaged Navier Stokes (RANS) models for CFD simulations:

- Conclusions on predictive capability of a turbulence model can only be determined with grid converged solutions.

$\circ$ When using wall functions, the first wall point should be held fixed while refining the grid.

- The computed overall vehicle drag is highly dependent on the choice of turbulent steady RANS model. Solutions may disagree with measurements by 0.5 to $50 \%$ for 0 degree yaw and by even higher percentages at yaw angles.

- Steady RANS models generally do a good job predicting the flow on the front and sides of the vehicle, where the flow stays attached and does not exhibit separation and recirculation zones.

- The flow structure in the trailer wake presented by the time-averaged experimental data does not compare with that computed with the steady RANS models. Thus, use of steady RANS to evaluate drag reduction devices in the trailer wake and tractor-trailer gap may provide inaccurate design guidance.

\section{Future Direction}

- Continue to develop and evaluate drag reducing conceptual designs and encourage and work with industry to road test the most promising drag reducing devices. Road tests with angled plates and SAE track tests with the pneumatic device are planned for FY04.

- Continue experimental data reduction and analysis for the generic conventional model (GCM). 
- Continue computations of flow around GCM, compare to experimental data, perform analyses, and provide guidance to industry on use of unsteady RANS and hybrid RANS/Large-Eddy Simulation methods.

- Develop and use an apparatus for studying wheel and tire splash and spray, pursuing ways to minimize this road safety hazard.

- Investigate air flow around rotating tires for improved brake cooling, as well as drag reduction.

- Investigate aerodynamics of filled and empty coal cars to determine effective concepts for drag reduction.

- Collaborate with DOE Industrial Consortium who will be conducting fleet tests of advanced aerodynamic drag reduction devices. Schedule industry site visits and meetings to share findings and encourage consideration of effective design concepts for road testing.

\section{Introduction}

A modern Class 8 tractor-trailer can weigh up to 80,000 pounds and has a wind-averaged drag coefficient around $\mathrm{C}_{\mathrm{D}}=0.6$. The drag coefficient is defined as the drag/(dynamic pressure $\mathrm{x}$ projected area). The higher the speed the more energy consumed in overcoming aerodynamic drag. At 70 miles per hour, a common highway speed today, overcoming aerodynamic drag represents about $65 \%$ of the total energy expenditure for a typical heavy truck vehicle. Reduced fuel consumption for heavy vehicles can be achieved by altering truck shapes to decrease the aerodynamic resistance (drag). It is conceivable that present day truck drag coefficients might be reduced by as much as $50 \%$. This reduction in drag would represent approximately a $25 \%$ reduction in fuel use at highway speeds. An estimated total savings of $\$ 1.5$ billion per year can be recognized in the United States alone for just a $6 \%$ reduction in fuel use. This reduction represents $1 \%$ of all fuel use in the United States.

The project goal is to develop and demonstrate the ability to simulate and analyze aerodynamic flow around heavy truck vehicles using existing and advanced computational fluid dynamics (CFD) tools. Activities also include an extensive experimental effort to generate data for code validation and a design effort for developing drag reducing devices. The final products are specific device concepts that can significantly reduce aerodynamic drag, and thus improve fuel efficiency, in addition to an experimental data base and validated CFD tools. The objective is to provide industry with clear guidance on methods of computational simulation and experimental modeling techniques that work for predicting the flow phenomena around a heavy vehicle and add-on drag reducing devices. Development of effective drag reducing devices is also a major goal.

The following reports on the findings and accomplishments for fiscal year 2003 in the project's three focus areas

- Drag reduction devices

- Experimental testing

- Computational modeling

A summary is given in the introduction portion of this report and detailed reports 
from each participating organization are provided in the appendices. Included are experimental results and plans by NASA, USC, GTRI, and LLNL in Appendices A through D. The computational results from LLNL and SNL for the integrated tractor-trailer benchmark geometry called the Ground Transportation System (GTS) model are in Appendices D and E, from ANL for the Generic Conventional Model (GCM, a.k.a. SLRT) in Appendix F, by LLNL for the tractor-trailer gap and trailer wake flow investigations in Appendix D, and turbulence model development and benchmark simulations being investigated by Caltech in Appendix G. USC is also investigating an acoustic drag reduction device that has been named 'Mozart' (Appendix B), GTRI

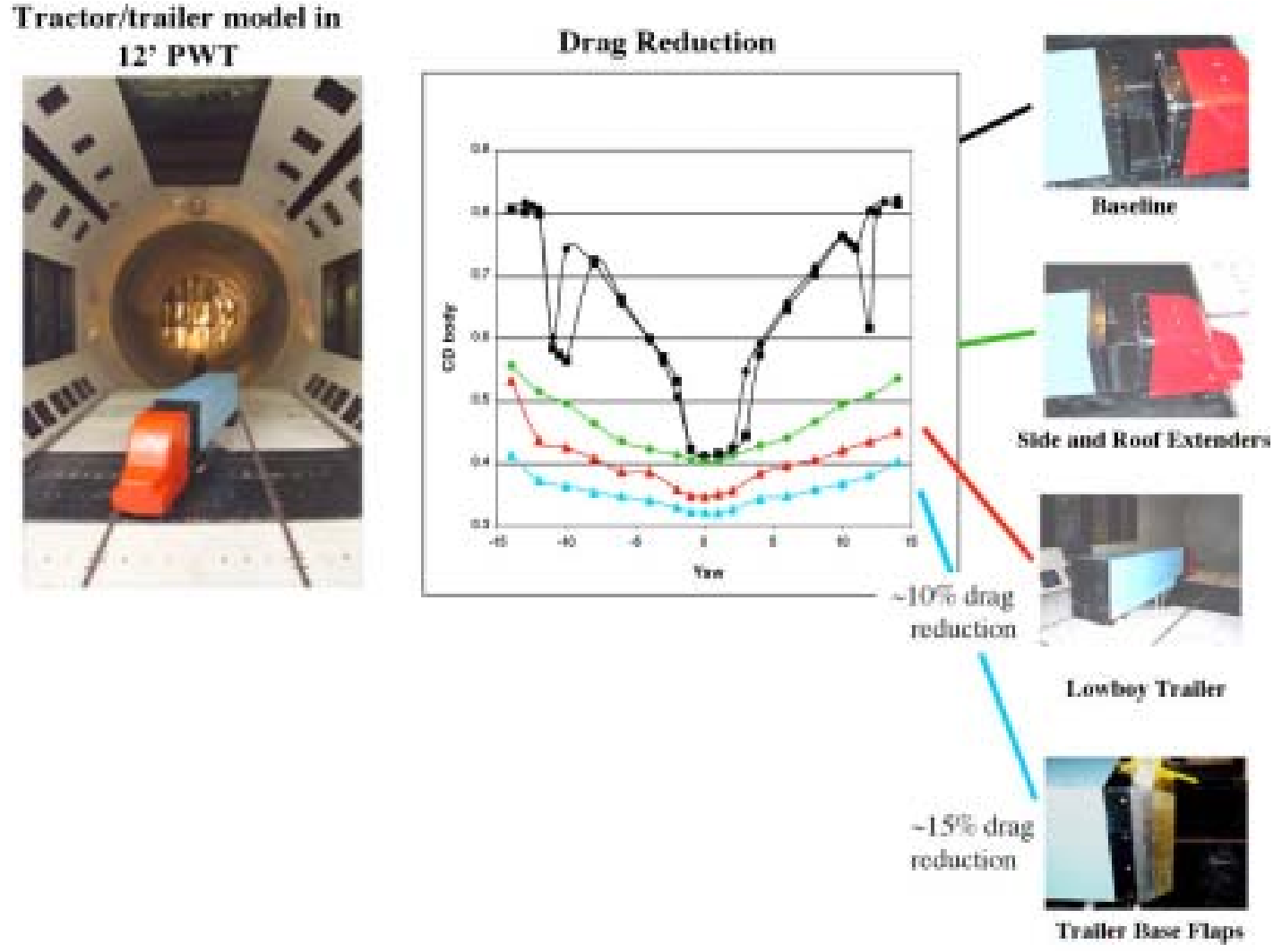

Figure 1. GCM in tunnel, variation in body drag coefficient with yaw angle for various configurations and device add-ons, and PIV and pressure sensitive paint results for baseline and with side extenders. 
The drag reduction for various device add-ons is shown in Figure 1, as a function of yaw angle. These results were obtained in the NASA Ames 12foot Pressure Wind Tunnel (PWT) using the realistic GCM geometry, tested at realistic highway Reynolds numbers. Side and roof extenders are shown to significantly reduce the drag and high yaw. Base flaps, as shown in a close-up in Figure 2, are expected to provide 50\% more drag reduction than boattails. For a tractor-trailer with a $C_{D}=0.55$ the percent drag reduction $\left(\Delta \mathrm{C}_{\mathrm{D}} / \mathrm{C}_{\mathrm{D}}\right)$ utilizing base flaps $(\sim 15 \%)$ and side skirts and/or a low boy $(\sim 10 \%)$ is estimated at 22 to 25 percent. Thus, the use of base flaps and skirts would provide an 11 to 12 percent fuel savings which should result in a $\$ 3$ billion per year fuel cost savings in the US. (Note that the cost of the device and possible maintenance over the year should also be considered for determining the overall cost savings to the fleet owner.)

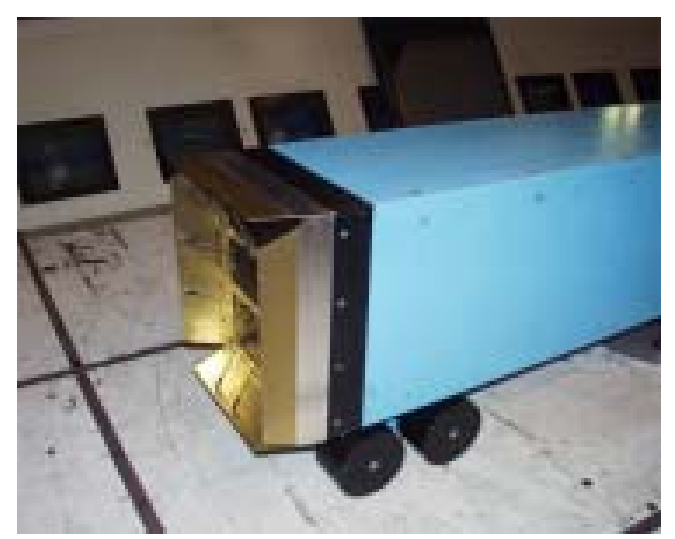

Figure 2. Base flaps (gold colored) mounted

on back end of trailer (blue) in NASA's 12-ft

pressure wind tunnel.

The base flaps are simple flat plates mounted on the edges of the back end of a trailer. The lengths of the plates match the dimensions of the trailer base (two $11.5 \mathrm{ft}$ long plates on the sides and two $8.5 \mathrm{ft}$ long plates on the top and bottom).
The width of the plates or how much they protrude from the trailer is about $1 / 4$ the width of the trailer or about 2 feet. Tilting the flaps about 20 degrees inward away from being flush with the trailer sides appears to provide the optimum drag reduction. The optimum flap angle for an on road vehicle is yet to be determined, but we expect it to be near 20 degrees.

Development has continued on tangential blowing aircraft-based technology to reduce $\mathrm{HV}$ drag by eliminating aft separation on the trailer and recovering base pressure on the back doors. Smaller-scale tunnel results have shown measured drag reductions as high as $15 \%$ due to blowing and $10-12 \%$ due to the device's corner rounding, for a total of 25 to $27 \%$. Blowing also has the potential to increase drag for use in braking, to reduce drag due to side winds, and to overcome directional instabilities due to side gusts. A maximum (best case) of 5-6\% fuel economy increase (not accounting for energy use for blowing) resulted during on-track HV fuel economy tests, and full-scale testing of a Pneumatic Sports Utility Vehicle is ongoing.

Road testing the drag reduction devices is needed to determine

- On road fuel savings,

- Optimal flap deflection angle for various tractor-trailer geometries,

- Optimal flap shape,

- Optimum skirt height,

- Durability, practicality, safety, ease of operation of proposed devices, and

- Impact on truck braking capability.

It is recommended that road testing include 
- Instantaneous broadcast fuel rate (1/2 second updates),

- Repeated forward and back trip runs over known, instrumented highways (e.g., South-to-North and North-toSouth runs over a portion of California I-15), and

- Base flap device evaluated in closefollowing combinations of 1 to 3 trucks.

To recognize these levels of fuel savings by the most effective use of drag reduction devices, the involvement and acceptance by tractor manufacturers, trucking associations, fleet owners, and drivers is critical. It is thus important to

- Solicit input and feedback from these organizations for design of base flaps and low boy and/or skirt construction,

- Demonstrate "actual" fuel savings from road tests and interest OEMs in doing testing, and

- Make site visits or attend DOE's Industry Consortium's Working Group meetings to encourage input and feedback.

Suggestions included encouraging the DOE Industry Consortium to road test base flaps and skirts or low boys as part of their DOE funded effort. Another suggestion is to contract with California Partners for Advanced Transit and Highways (PATH) to perform the proposed road tests as part of their 3truck demonstration platoon.

\section{Experimental Findings and Suggested Guidance}

Experiments have been conducted on a Generic Conventional Model (GCM) in the NASA Ames 7-ft x 10-ft wind tunnel for Reynolds numbers (Re) of 1 million based on the width of the trailer, which corresponds to a full-scale vehicle traveling at roughly 15-mph. Experiments have also been performed on the GCM geometry in the NASA Ames 12-ft pressure wind tunnel (PWT) for Re of 1 and 6 million, where the later corresponds to a full-scale vehicle traveling at 80-mph. Geometry configurations included the addition of tractor side extenders, a low boy trailer, and boattails and angled flaps on the trailer's trailing edge. The results in the PWT are obtained for a constant Mach number $(\mathrm{Ma}=0.15)$ by pressurizing the tunnel. This allows for the determination of Re and geometry effects. Yaw angles were varied from +14 to -14 degrees measured from the vehicle length axis and wind direction so that accurate wind-averaged drag could be determined, in addition to determining the effect of yaw angle. The following is a list of experimental techniques and measurements:

- Internal balance measured the vehicle forces and moments

- Load cells measured the drag for the body axis and yawing moment of the tractor

- Static pressure taps on the model (476) and taps on the walls and floor (368) measured static pressure conditions

- Unsteady pressure transducers (14) provide a pressure time history on the surface of the vehicle

- Three-dimensional particle image velocimetry (PIV) provided a time history of the velocity field on planes in the wake of the vehicle and in the tractor-trailer gap.

Drag measurements alone are not sufficient to provide an understanding of the impact of geometry modifications and direction for design improvements. It is recommended that advanced 
measurement techniques like PIV and pressure sensitive paint (PSP) be included. These advanced techniques provide important information on the global and local structure of the flow and can provide clear design direction.

The following are the determined $\mathrm{Re}$ effects (note: Re is based on the width of the trailer and freestream velocity):

- Re effects on $C_{D}$ are in general minimal for experiments with $\mathrm{Re}$ above 1 million. This finding supports the common use of scaled down vehicles and $\mathrm{Re}$ below typical highway Re for experimentation.

- It should be noted that some Re influence was apparent on the flow structure in the tractor-trailer gap and the back end of the trailer. It was most apparent in the upper portion of the flow region in the gap and in the wake. Thus, some inaccuracies should be considered when evaluating gap and wake drag reduction devices at lower than highway Re. Low Re experiments should provide ball park estimates, but accurate optimization of devices may require road testing.

- Edge radius effects and/or the cleanliness of the vehicle upstream flow are critical to achieving accurate predictions. Corner radii on the leading edge of the vehicle should provide $\operatorname{Re}>50,000$, based on corner radius and tunnel freestream velocity. Tripping the flow at the vehicle leading edge may also be required to avoid flow separation.

\section{Computational Findings and Suggested Guidelines}

Team members from LLNL, SNL, ANL, and Caltech are investigating a wide range of turbulence models including steady and unsteady Reynolds-averaged
Navier-Stokes (RANS and URANS, respectively), large-eddy simulation (LES), and hybrid methods that use a combination RANS and LES models in the simulation. In addition, various numerical approaches are being considered including finite volume, finite element, and vortex methods. The focus of the focus of this years effort was steady RANS with and without the use of wall functions. Wall functions provide an approximation to the flow field in the wall region and the flow field is not resolved.

The following are the general observations and guidelines for steady RANS modeling:

- Conclusions on predictive capability of a turbulence model can only be determined with grid converged solutions. Predicted flow structures in separated regions, like the trailer wake, vary significantly with grid refinement. Variation in overall drag is not substantial but still apparent with grid refinement.

- When using wall functions, the first wall point should be held fixed while refining the grid (i.e., the distance from this grid point to the wall should not change), but it is appropriate to decrease the width of the wall elements while refining the grid (i.e., refinement in direction tangent to walls).

- The computed overall vehicle drag is highly dependent on the choice of turbulent steady RANS model. Solutions may disagree with measurements by 0.5 to $50 \%$ for 0 degree yaw and by even higher percentages at yaw angles. Thus, the performance of steady RANS models for a given geometry is not predictable and experimental results 
to determine ball park accuracy is critical when relying on steady RANS for design guidance.

- Steady RANS models generally do a good job predicting the flow on the front and sides of the vehicle, where the flow stays attached and does not exhibit separation and recirculation zones.

- The flow structure in the trailer wake presented by the time-averaged experimental data does not compare with that computed with the steady RANS models. The trailer wake is a region of transient full flow separation and large recirculation zones. Thus, use of steady RANS to evaluate drag reduction devices in the trailer wake and tractor-trailer gap may provide inaccurate design guidance.

Near term plans are to organize similar types of guidelines related to the performance of unsteady RANS, LES, and hybrid models.

\section{Conclusions}

The DOE Heavy Vehicle Aero Drag Team has successfully modeled the flow field around a generic conventional model both experimentally and computationally. This effort has provided detailed insight into the flow phenomena, which has lead to the successful development of drag reduction devices. Evaluation of an angled plate base drag device (15\%) with the use of a low boy trailer and/or side skirts $(10 \%)$ indicates an expected drag reduction of 22 to 25 percent. Use of these devices should provide an 11 to 12 percent fuel savings which is estimated to result in a $\$ 3$ billion per year fuel cost savings in the US. Efforts continue on investigation of acoustic and pneumatic devices to reduce base and separation drag, and some new ideas for reducing tractor-trailer gap drag. Future new areas being investigated are wheel and wheel well aerodynamics related to brake cooling, tire splash and spray, and an entire new related area of investigation involving the evaluation of coal car aerodynamics with the objective of identifying drag reduction devices for filled and empty cars. 


\section{APPENDIX A}

\section{Experimental Modeling of Generic Conventional Model}

Principal Investigator: J. Ross

Co-Investigators: D. Satran, J.T. Heineck, S. Walker, and D. Yaste

NASA Ames Research Center

MS 260-1, Moffett Field, CA 94035

(650) 604-6722; jcross@mail.arc.nasa.gov

Technology Development Manager: Sid Diamond

202-586-8032, sid.diamond@ee.doe.gov

Technical Program Manager: Jules Routbort

630-252-5065,routbort@anl.gov

Contractor: NASA Ames Research Center

Contract No.: DE-AI01-99EE50559

\section{Objective}

- Acquire data for calibrating CFD codes used to predict the performance of generic vehicles representing Class 8 tractor-trailers.

- Evaluate drag reduction devices.

\section{Approach}

- Perform experiments utilizing standard and advanced measurement techniques in the NASA Ames 7-ft x 10-ft wind tunnel and 12-ft Pressure Wind Tunnel on the Generic Conventional Model (GCM).

- Include evaluation of side and roof extenders, boattail plates, base flaps, trailer skirts, and lowboy trailer configurations.

\section{Accomplishments}

- Two test entries were completed in the 12-Foot Pressure Wind Tunnel to obtain the aerodynamic data on the GCM.

- Limited Reynolds number effects were found for the GCM.

- Base flaps were the most effective drag reduction device for trailers.

- Trailer skirts produced some drag reduction but the lowboy trailer configuration was more effective.

- Side and roof extenders were effective for the tractor. 


\section{Future Direction}

- Document the test results and publish the database for computational code validation support.

- Begin experimental efforts for the evaluation of wheel and wheel well aerodynamics for brake cooling and reduction of splash and spray.

- Begin experimental efforts for the evaluation of coal car aerodynamics.

\section{Introduction}

In cooperation with the Department of Energy, a series of wind tunnel tests have been conducted by the Experimental Aerophysics Group at NASA Ames Research Center on generic vehicles representing Class 8 tractor-trailers. The primary goal for these wind tunnel tests was to produce high quality data for validating Computational Fluid Dynamics (CFD) codes. The final test was conducted in the NASA Ames Research Center's 12Foot Pressure Wind Tunnel generating data at a variety of Reynolds Numbers, from $1 / 8$ to full-scale road values, on the same $1 / 8^{\text {th }}-$ scale model.

Current CFD codes have a difficult time accurately predicting the drag levels for tractor-trailer configurations. Enhanced CFD codes that can reliably produce accurate results will enable industry to better understand all aspects of the flow around trucks, leading to better integrated tractor/trailer designs. To properly validate the CFD codes, the aerodynamic databases include vehicle forces and moments, surface pressures, fluctuating pressures on the rear of the tractor and front and rear of the trailer, skin friction measurements, and 3D Particle Image Velocimetry (PIV) offbody flow field measurements in the gap between the tractor and trailer and in the wake behind the trailer.
Besides generating a database for validating CFD codes, the Experimental Aerophysics Group has investigated numerous drag reduction devices. Some examples of the devices include the side and roof extenders which reduce the gap between the tractor and trailer, the lowboy trailer configuration where the ground clearance is reduced to a minimum and the wheels are covered by fairings, and base flaps which help turn the flow inward as it leaves the back of the trailer reducing the size of the wake and providing increased base pressure. Additional drag reduction concepts included boattail plates, gap fairings, and trailer skirts.

\section{$\underline{\text { Results }}$}

As can be seen in Figures 1A through $5 \mathrm{~A}$, significant drag reduction is produced with the different devices. The basic truck geometry produced high levels of drag at yaw angles greater than 4 degrees. The increase in drag was eliminated with the addition of the side and roof extenders. The lowboy trailer produced drag reduction on the order of 10 percent and was comparable to the boattail plates. The base flaps were the most successful trailer modification and produced drag reduction on the order of 15 percent. If the lowboy trailer is combined with the boat tails or base flaps, the results are additive with a drag reduction of over $20 \%$. Although there 
were some Reynolds number effects, for the most part the effects were small and results were adequately predicted using data generated at lower Reynolds numbers.

A significant portion of the effort was expended in gathering the PIV data. This was the first time a PIV system was used in a pressurized facility, which made a complex system even more complicated [1]. Although the 12-foot wind tunnel has better optical access than most wind tunnels, the access that was available created additional challenges for the PIV system. Significant changes in the flow field between the tractor and trailer are documented by the PIV system in Figure $1 \mathrm{~A}$ with and without the rear side extenders. The addition of the roof extender provided a slight improvement in the drag reduction to that provided by the side extenders.

\section{Heavy Vehicle Aerodynamics and Drag Reduction}

Tractor/trailer model in

\section{2' PWT}

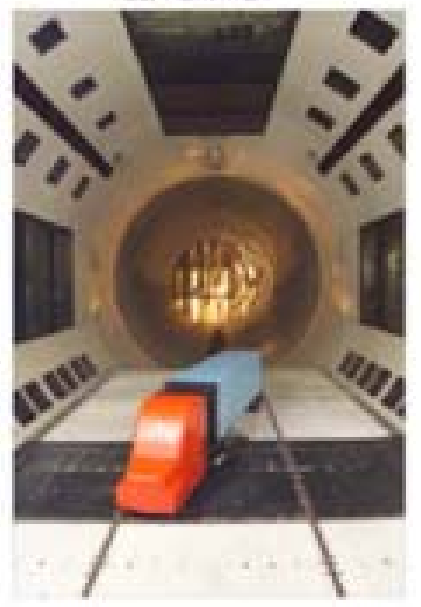

Surface Pressures \& PIV Velocity Contours

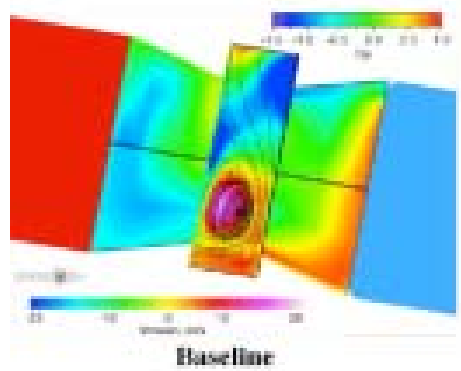

\section{Drag Reduction}

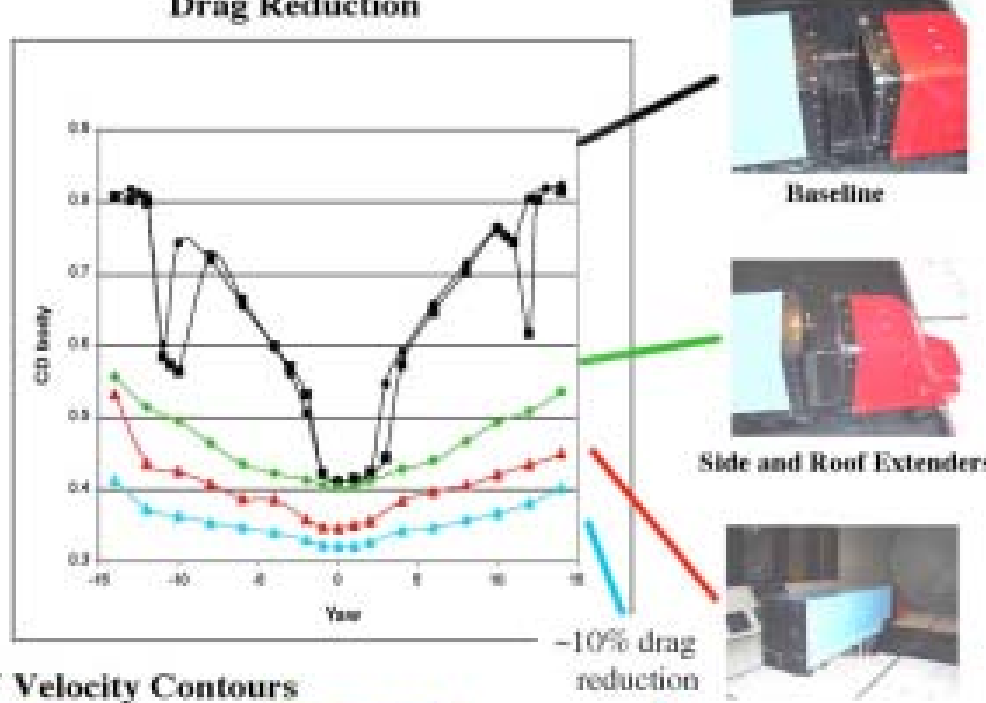

in 12 in in 80 is

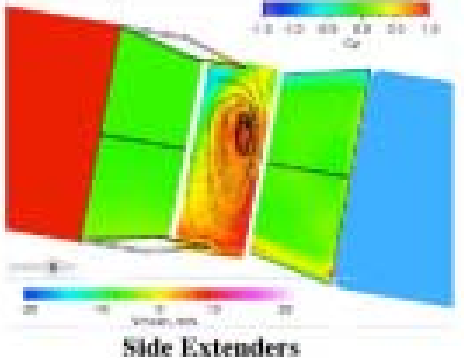

Lewby Trailer

$\sim 15 \%$ dragt reduction

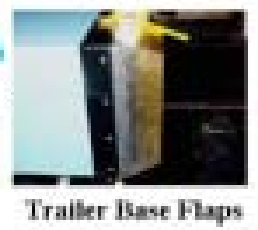

Figure 1A. GCM in tunnel, variation in body drag coefficient with yaw angle for various configurations and device add-ons, and PIV and pressure sensitive paint results for baseline and with side extenders. 


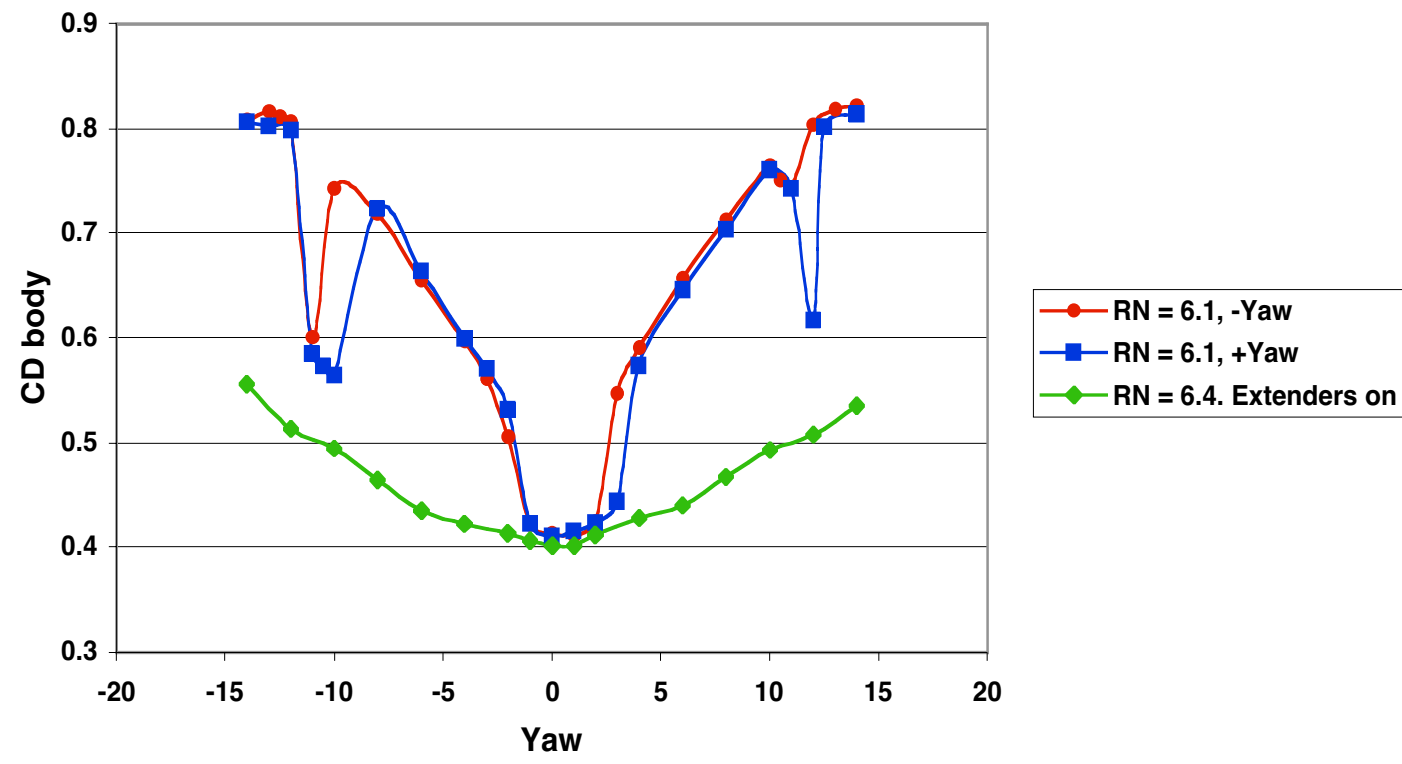

Figure 2A. Drag coefficient versus yaw angle with and without side extenders for $\operatorname{Re}=6$ million.

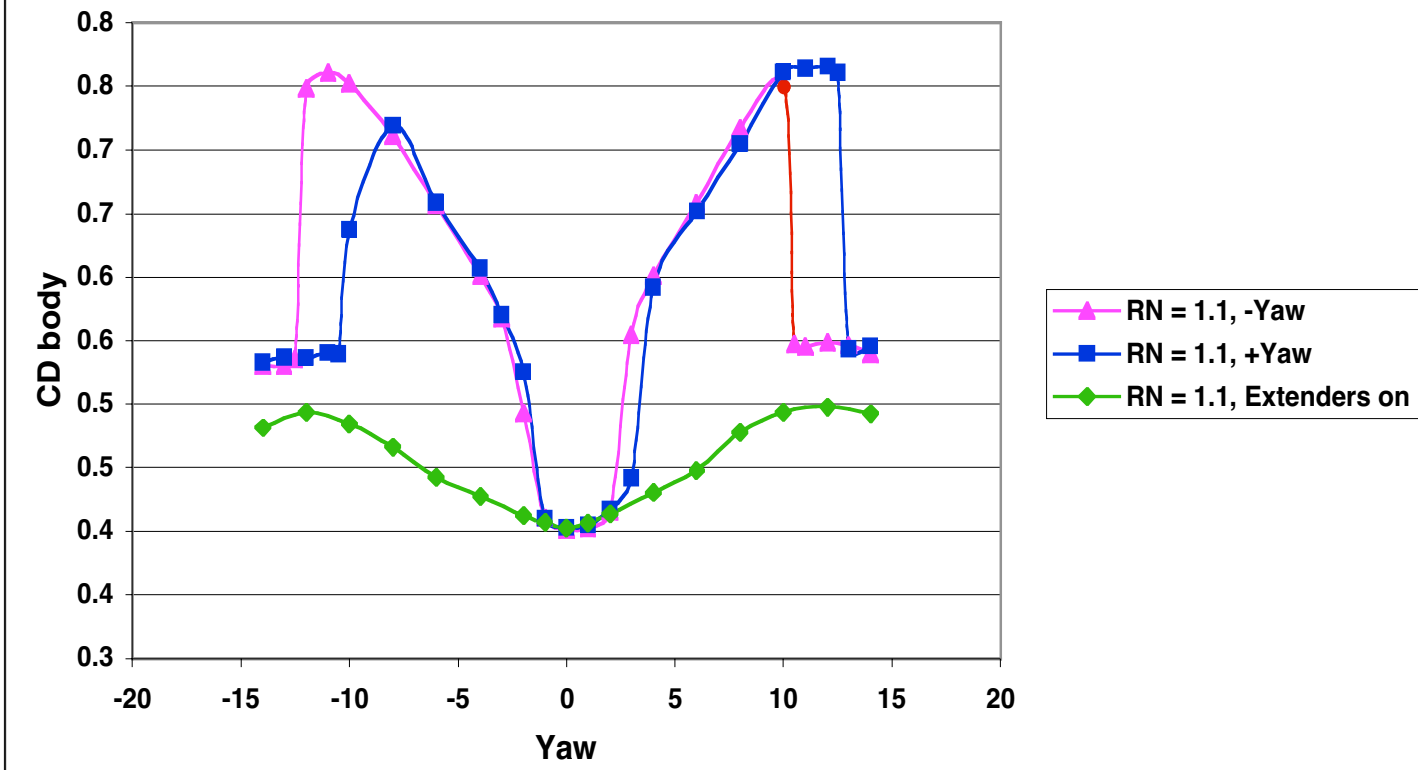

Figure 3A. Drag coefficient versus yaw angle with and without side extenders for $\operatorname{Re}=1.1$ million. 


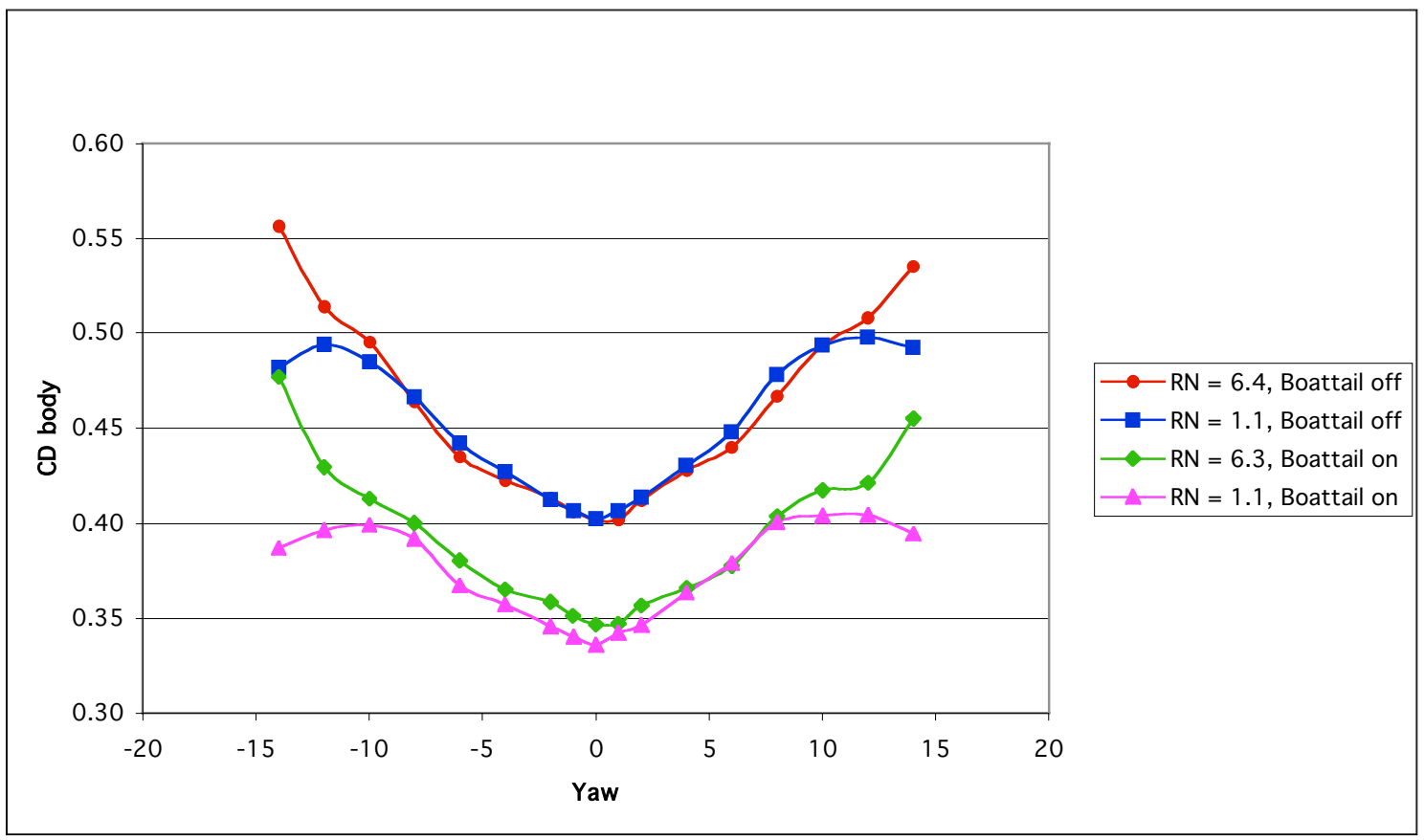

Figure 4A. Drag coefficient versus yaw angle with and without boattail plates for $\operatorname{Re}=1.1$ and 6 million.

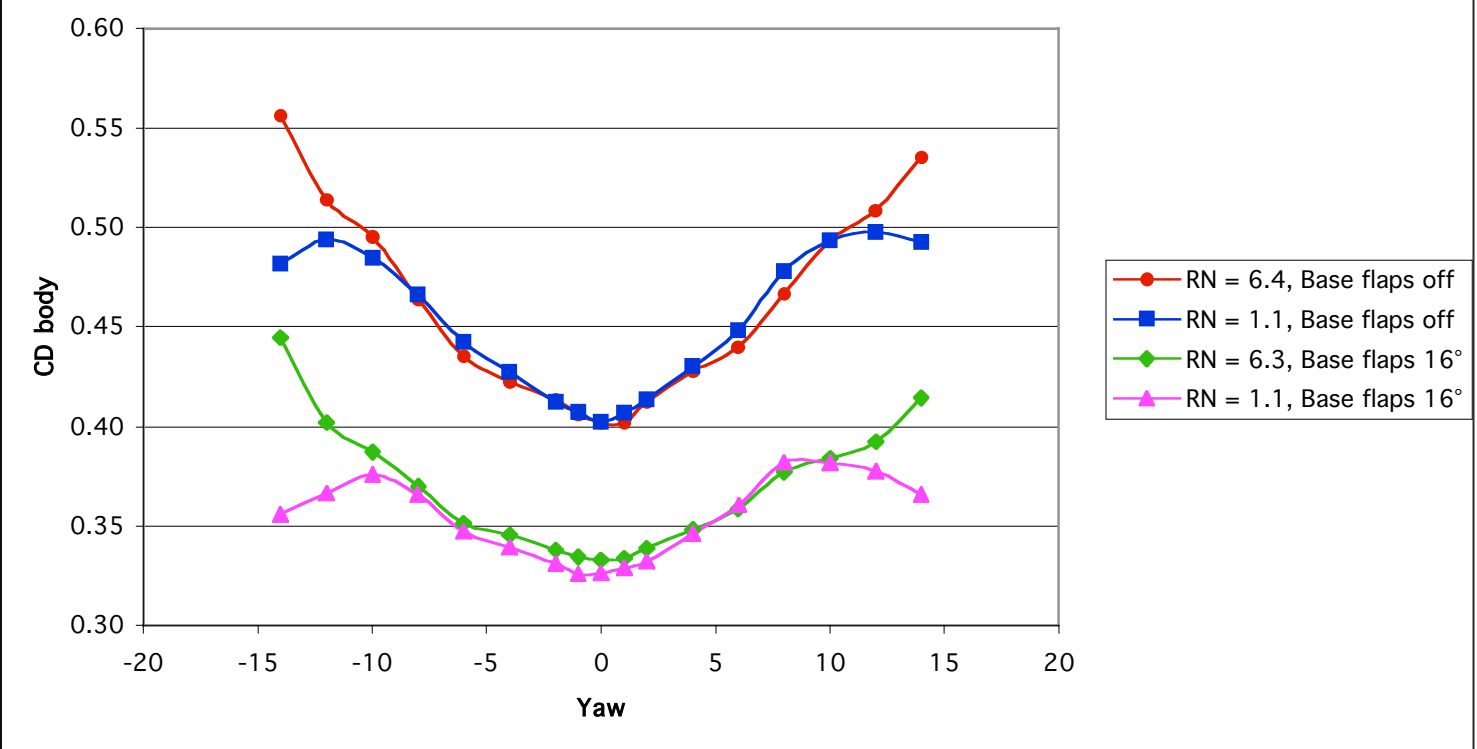

Figure 5A. Drag coefficient versus yaw angle with and without base flaps for $\operatorname{Re}=1.1$ and 6 million. 


\section{Conclusions and Future Activities}

Two test entries were completed in the 12Foot Pressure Wind Tunnel to obtain the aerodynamic data on the GCM. The results indicated limited Reynolds number effects. The base flaps were the most effective drag reduction device for trailers tested. Trailer skirts produced some drag reduction but the lowboy trailer configuration was more effective. Side and roof extenders were effective drag reducers for the tractor.

Future activities planned for FY04 include documentation of the test results and publish the database for computational code validation support, begin experimental efforts for the evaluation of wheel and wheel well aerodynamics for brake cooling and reduction of splash and spray, and begin experimental efforts for the evaluation of coal car aerodynamics.

\section{$\underline{\text { References }}$}

[1] J.T. Heineck, S.M. Walker, D.M. Yaste, "The Development of a 3C-PIV System for the 12-Foot Pressure Tunnel at NASA Ames Research Center," 20th International Congress for Instrumentation Aerospace Simulation Facilities (ICASF), DLR Gottingen, August 25-29, 2003. 


\section{APPENDIX B \\ Experimental Measurement of the Flow-field of Heavy Trucks}

Principal Investigator: F. Browand

Co-Investigators: M. Hammache, T.-Y. Hsu

Aerospace \& Mechanical Engineering, University of Southern California

RRB 203, Los Angeles CA 90089-1191

(213) 740-5359; fax: (213)740-7774; e-mail:browand@spock.usc.edu

Technology Development Manager: Sid Diamond

202-586-8032,sid.diamond@,ee.doe.gov

Technical Program Manager: Jules Routbort

630-252-5065,routbort@anl.gov

Contractor: DOE Oakland Operations Office, Berkeley Site Office

Contract No.: DE-AC03-98EE50512

\section{Objective}

- Improve the performance of heavy trucks by reducing aerodynamic drag.

\section{Approach}

- Produce a smaller truck wake and a lower truck drag by providing for active flow control at the base of the trailer utilizing an arrangement of base flaps.

- Evaluate the importance of the gap between tractor and trailer in producing drag.

\section{Accomplishments}

- Wind tunnel tests of flow control device at trailer base document improvements in drag of $\Delta \mathrm{C}_{\mathrm{D}}=.06-.08$, or about $13-14 \%$ for a modern truck.

- Wind tunnel flow field studies document the appearance of violent cross-gap flows under certain conditions. The cross-gap flow separates the flow along the side of the trailer and increases drag. Suggestions have been made to minimize this unwanted cross-gap flow.

\section{Future Direction}

- Test for additional drag saving in wind tunnel models by increasing the strength of the active flow control.

- Perform over-the-road testing to verify drag savings for the base flap device.

- Initiate a program and a new testing apparatus to study wheel/tire splash and spray. 


\section{Introduction}

This report describes the progress we have made on two separate aerodynamic problems (i) reducing the base drag of a tractor-trailer with a combination of passive and active control devices, and (ii) describing the sensitivity of the drag to the geometry of the gap between tractor and trailer. Included in this report is a description of work we have completed, and work we will continue into FY'04.

\section{Reducing Base Drag}

There are two general approaches for the reduction of base drag. One approach, we would term passive control, is to alter the geometry of the base region in some way. Cooper (1985) shows that the drag coefficient for a typical straight-sided truck at 0.78 can be reduced to a value on the order of 0.72 by the use of thin plates attached along the edges of the trailer base. Further, Cooper demonstrates that the effectiveness of the drag reduction increases with nondimensional flap length, $l$, defined as $l=$ $\mathrm{L}_{\mathrm{f}} / \operatorname{sqrt}(\mathrm{A})$, where $\mathrm{L}_{\mathrm{f}}$ is the flap length and $\mathrm{A}$ is the cross-sectional area of a truck. He points out that most of the drag reduction is accomplished for flap length less than a value of approximately, $l=0.18$. Flaps such as those studied by Cooper can be seen in Figure 1B installed on a model truck test in a wind tunnel at NASA Ames.

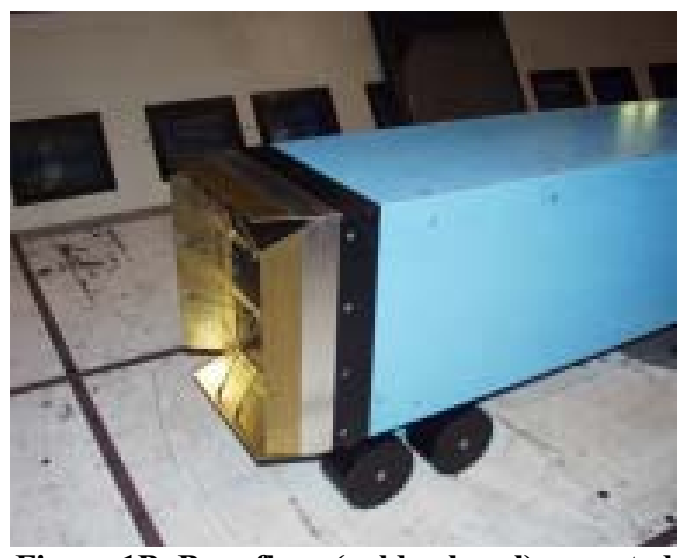

Figure 1B. Base flaps (gold colored) mounted on back end of trailer (blue) in NASA's 12-ft

pressure wind tunnel.

The second approach is to seek additional drag reduction by means of an active forcing (such as an oscillating flap, or a blowing slot), meant to alter the boundary layer properties - usually to avoid an unwanted separation. Nishri and Wygnanski (1998) show that the effectiveness in delaying flow separation is determined by the location of the jet, the frequency of the induced oscillation, the net momentum flux coefficient, and the shape size of the slot. The frequency of the induced oscillation, $f$, is nondimensionalized by the flap length and free-stream velocity, $\mathrm{U}_{\infty}$. It is defined as $\mathrm{F}^{+}=\frac{\mathrm{f}^{*} \mathrm{~L}_{\mathrm{f}}}{\mathrm{U}_{\infty}}$. The net oscillatory momentum flux coefficient, $\mathrm{C}_{\mu}$, is defined as $\mathrm{C}_{\mu}=2 * \frac{\mathrm{g}}{\mathrm{L}_{\mathrm{f}}} *\left(\frac{\mathrm{U}_{\mathrm{j}}}{\mathrm{U}_{\infty}}\right)^{2}$, where $\mathrm{g}$ is the slot height, i.e., the gap between the flap and the side wall, and $U_{j}$ is the amplitude of the oscillatory jet fluctuation. Favorable delays in separation are obtained for slot heights on the order of $1 / 3-1 / 2$ of the incoming boundary layer displacement thickness, and for $C_{\mu}$ values less than $0.1 \%$. The most effective non-dimensional 
frequencies appear to lie in the range $\mathrm{F}^{+}$ $=0.3-1.5$.

Our approach encompasses both a modification of the base geometry by means of the addition of flat-panel flaps, and an additional active control by means of an oscillatory mass flow perturbation within the boundary layer meant to delay flow separation over the surface of the flaps. The (flat) flaps are attached to the trailer base along the trailer base edges, and are inclined to the free stream to close the wake more efficiently.

The experiments utilizing oscillatory perturbations are conducted in the Dryden wind tunnel at the USC Ground Vehicle Aerodynamics Laboratory. A roughly $1 / 15$ scale model resembling a trailer is utilized for the study (see Figure 2B). The model is fitted with a shaped nose-piece to ensure attached flow over the forward portion of the model. The model is equipped with a force balance to measure drag. The Reynolds numbers (based on the squareroot of the model cross-sectional area), range from $0.1 \times 10^{6}$ to $0.4 \times 10^{6}$. A sine wave is chosen as the forcing function. The forcing frequency is zero or within the range $40-600 \mathrm{~Hz}$. The corresponding non-dimensional frequencies are, $\mathrm{F}^{+}=0,0.17-3.93$. The oscillatory momentum flux coefficient, $\mathrm{C}_{\mu}$, ranges from 0 (no forcing) to $2 \%$.

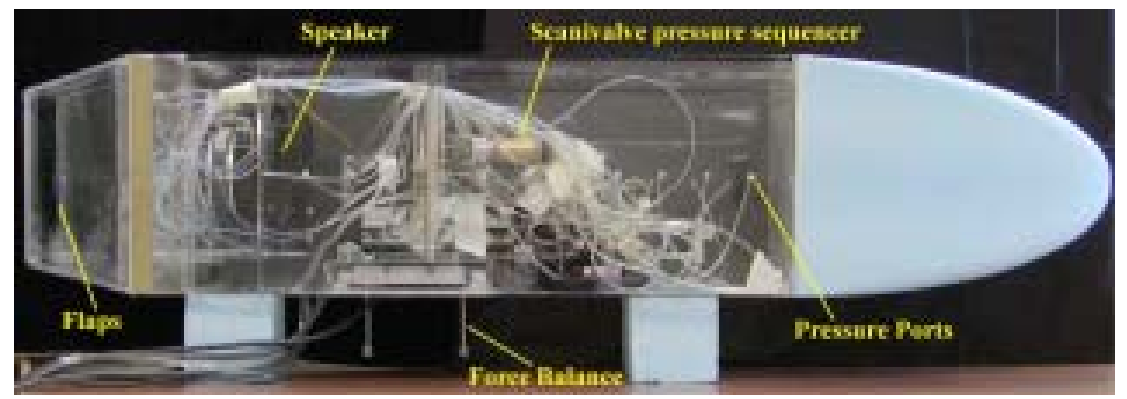

(a)
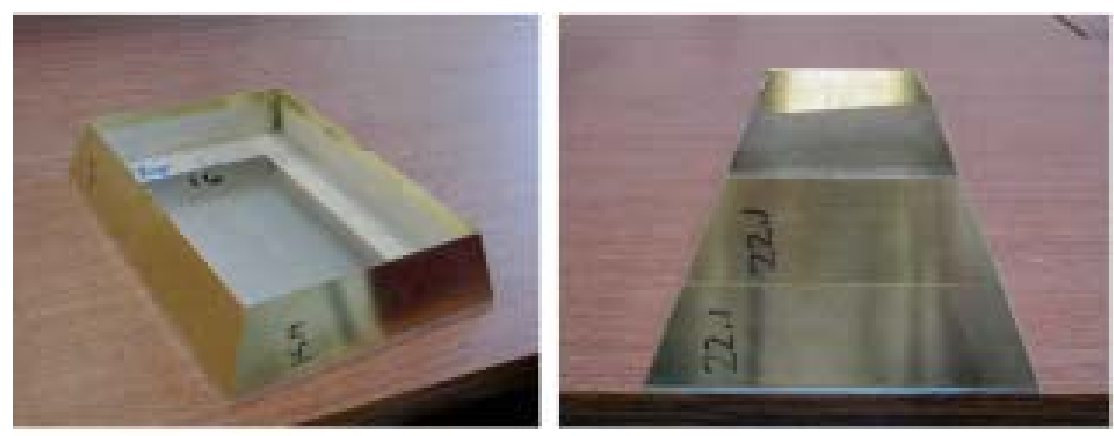

(b)

Figure 2B. USC model equipped with rounded nose and base flaps 
The present study has found that a simple, passive base-flap deflection-no forcing-produces significant drag saving. The effect of flap angle upon drag reduction is shown in Figure $3 \mathrm{~B}$. The horizontal axis represents the flap angle in degrees, and the vertical axis represents the change in drag coefficient, $\Delta \mathrm{C}_{\mathrm{D}}=\mathrm{C}_{\text {Dnoflaps }}-\mathrm{C}_{\text {Dflaps, }}$. A positive value of $\Delta \mathrm{C}_{\mathrm{D}}$ corresponds to a reduction in drag. Data is shown here from three widely different experiments-one performed in our wind tunnel, one test recently performed in the 12-foot wind tunnel at NASA Ames, and Cooper's original results. All of the $\Delta C_{D}$ curves have a roughly similar shape. Drag savings first increase and then decrease with increasing flap angle. Thus there is an optimum angle for maximum drag saving. The maximum saving is in the range $\Delta C_{D} \approx .06-.08$ for all three experiments. The three separate tests are accomplished in three different wind tunnels using models of different geometry. The data thus suggest a robust drag saving that is neither particularly dependent upon the details of truck shape nor upon the Reynolds number of operation. However, the angle for maximum saving does seem to depend upon Reynolds number. The optimum angle seems to increase steadily from 9-12 degrees at $0.3 \times 10^{6}$ to perhaps 20 degrees at $6 \times 10^{6}$.

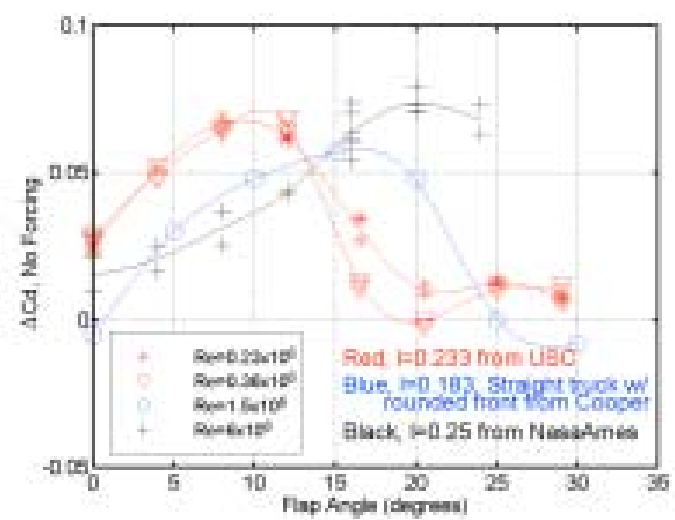

Figure 3B. Base drag improvements, expressed as a change in drag coefficient for

three separate experiments.

Now consider the addition of oscillatory blowing/suction along the edges of the model base, coincident with the origin of the flap. The purpose of the oscillatory blowing/suction is to attempt to maintain attached flow over the flap for larger flap deflection angles, and thereby to further reduce drag. Figure 4B summarizes the best of our results. The drag coefficient difference, $\Delta \mathrm{C}_{\mathrm{D}}$, is shown for the short flap length - with and without oscillatory blowing. The experimental reliability is indicated by the scatter of data points representing multiple trials. Blue curves are the noforce cases. The results in red, for a momentum coefficients of $\mathrm{C}_{\mu}=0.15 \%$ and $2 \%$, demonstrate the effectiveness of oscillatory blowing/suction. There is a modest additional drag saving at the higher flap angles, but the application of forcing has very little effect in the vicinity of peak saving. Thus the peak saving is almost unchanged. The cause of this unexpected result is still under investigation. Examination of the forced and unforced results in the vicinity of 12 degrees suggests that the momentum addition does delay the onset of separation in some manner. However, we had anticipated that in the presence 
of forcing and delayed separation, the drag saving would continue to increase beyond the unforced peak at 9-12 degrees. Rather, the demonstrated effect of forcing is to broaden the peak region by extension to larger angles but not to increase the maximum value of drag saving.

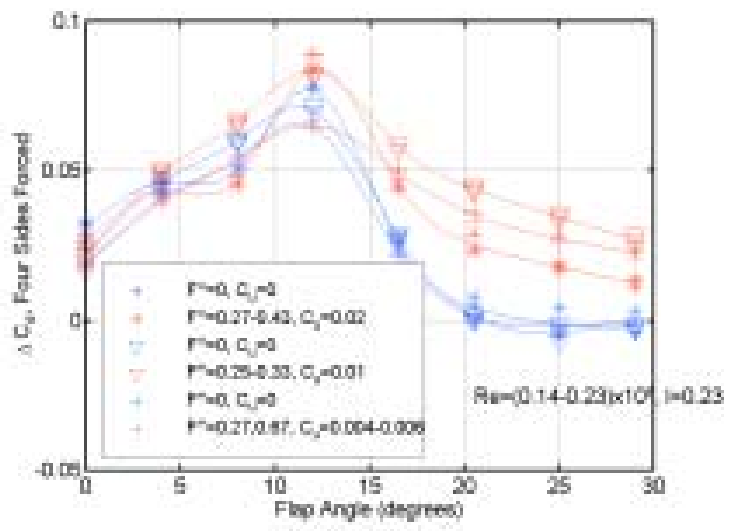

Fig. 4B Effect of oscillatory forcing on drag

decrease.

The optimum forcing frequency definitely lies in the range $\mathrm{F}^{+} \approx 0.27$ that will effect flow modification, but that forcing amplitude is critical. We begin to see noticeable change only at momentum coefficients on the order of $10^{-3}$ or greater $\left(C_{\mu} \geq 0.1 \%\right)$. Nishri \& Wygnanski (1998) show observable increases in lift over deflected flaps at momentum coefficients as small as $0.01 \%$. Evidently drag reduction for a three-dimensional body such as ours is a much more subtle proposition. The present results also show that oscillatory momentum addition has little effect on drag reduction unless the net oscillatory momentum flux coefficient is equal or greater than $0.1 \%$. Increasing the oscillatory momentum perturbation to a coefficient value of $2 \%$ produces drag savings at angles greater than 9-12 degrees, but has very little effect upon the maximum saving at 9-12 degrees.

We are presently performing DPIV studies (Digital Particle Image Velocimetry) of the flow adjacent to the flap with and without the oscillatory acoustic/blowing. The intent is to answer the question regarding the state of the boundary layer and the degree of attached/unattached flow present. The cases studied to date are the 0 and 12 degree flap angles. For the zero degree flaps angle, the wake profiles obtained from the ensemble average of 350 image pairs suggest that there is no difference between forcing and non-forcing.

The preliminary data also suggests that the flow is attached up to flap angle equal to 12 degrees. However, there is a small drag reduction at a 12 degree flap angle (see Figure 4B), and this drag reduction is reflected in the flow over the flap as seen by the detailed flow field (DPIV) map shown in Figure 5B. Velocity contours are shown in the wake region just downstream from the flap — blue for the unforced case, and red for the forced. The suggestion is that the wake is slight more narrow when oscillatory forcing is present.

Follow-on experiments are planned to investigate a larger range of forcing amplitudes, and a variety forcingfunction duty cycles. In addition, DPIV will be used to capture the detailed flowfield in the vicinity of the flap for all cases. 


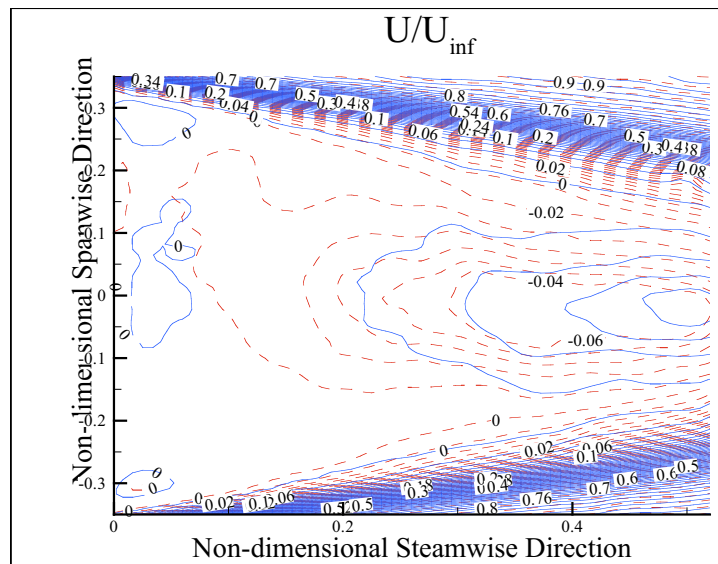

Fig. 5B Contour plot of free-stream velocity in the wake region.

Drag Rise Due to the Geometry of the Gap Between Tractor and Trailer The second part of this report summarizes the wind tunnel experiments on the aerodynamics of tractor-trailer models to show that the drag on the model is sensitive to the width of the tractor-trailer gap $(\mathrm{G})$, and to the angle of yaw with respect to wind direction. The measurements are performed at a wind tunnel speed of approximately 26 $\mathrm{m} / \mathrm{s}$. The model Reynolds number for the tests, based upon the square root of the truck cross-sectional area, $\sqrt{ } \mathrm{A}$, is about 310,000 . Figure $6 \mathrm{~B}$ shows both tractor and trailer resting on an interior turntable in the ground plane. The turntable allows the models to be yawed with respect to the flow direction.

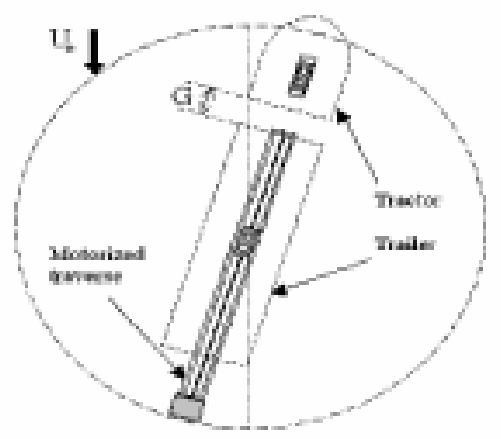

Fig. 6B. Detail of turntable and traverse mechanism inside ground plane.
The drag on the tractor and trailer, at zero-yaw, as a function of gap width are plotted in Figure 7B. In the range of $\mathrm{G} / \sqrt{ } \mathrm{A}$ of 0.1 to 0.5 , the trailer experiences less drag than the tractor, due to the shielding effect. As the gap opens up beyond approximately 0.5 , the total drag rises rapidly to large values - most of the increased drag is attributable to the trailer.

The DPIV measurement technique is adopted in this experiment to visualize and quantify the flow in the gap area. Whole-field velocity measurements are obtained for various combinations of gap width and angles of yaw, though the present discussion is limited to zero-yaw cases. A total of 350 instantaneous realizations are acquired for each combination of gap and yaw, and for three horizontal planes within the gap-a total of 36 combinations. Changes of the flow structure within the gap region are investigated by performing various forms of conditionalaveraging.

At low gap width, typically below $\mathrm{G} / \sqrt{ } \mathrm{A}$ $\sim 0.5$, the flow in the gap is turbulent, but steady in a broad sense-consisting of a re-circulating in the form of a torus. As the gap increases, the flow in the gap becomes highly unsteady with large cross-gap flows that alternate from side to side. An example of this variability is demonstrated in Figure 7B. A certain fraction of the time, the flow behavior is described by the left-hand portion of the picture labeled symmetric. At other times, the flow exhibits a highly asymmetric pattern and exits from one side of the gap. The mirror image of this state is also seen, although it is not illustrated here. These highly asymmetric flow states produce large 
wake regions along the sides of the trailer, and signal the drag rise onset seen in Figure 7B.

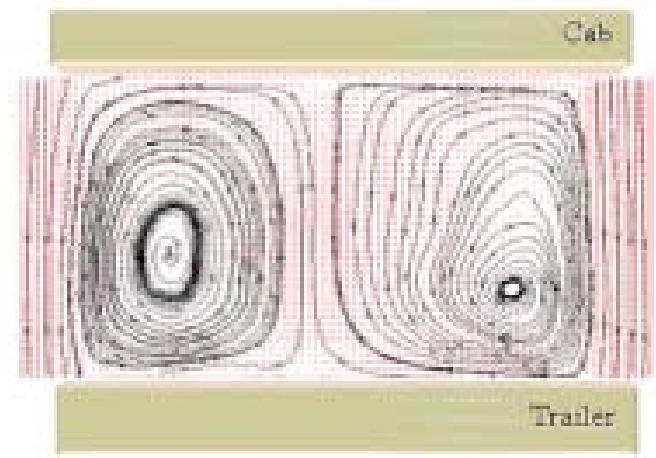

Symmetric tiow

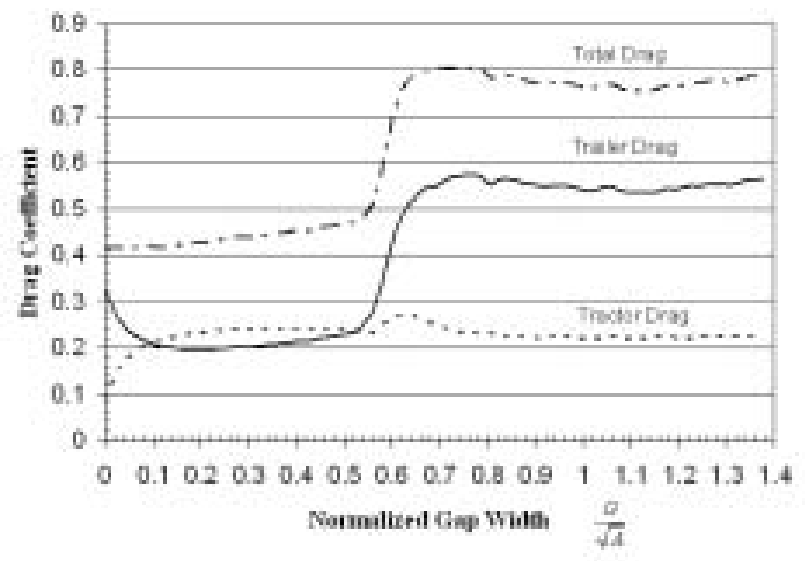

Fig. 7B. Drag coefficient versus gap length.

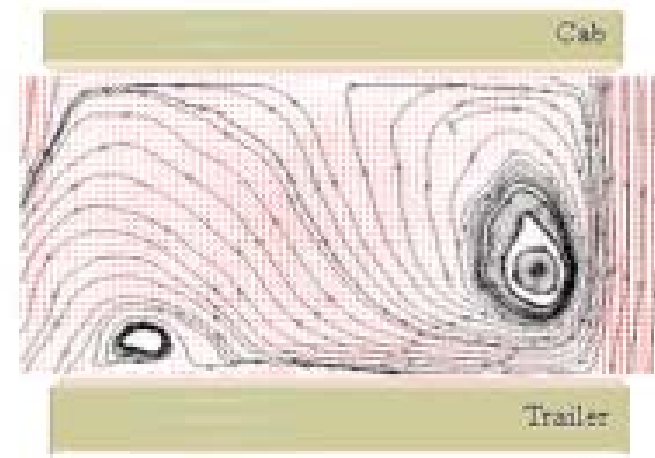

Asymmetric flow

Fig. 8B. Ensemble-averaged streamline patterns of the flow in the gap at zero yaw, $G / \sqrt{A}=0.55$. (a) symmetric flow, (b) asymmetric flow.

The final figure describes the drag savings that can be realized by arranging two truck-like models in a tandem configuration. The truck-like models are either "blunt" or "rounded". Separately, the truck shapes have drag coefficients of approximately 0.94, and 0.51, respectively. Four tandem combinations are possible from the two shapes, as depicted in Figure 9B. The quantity plotted in Figure 9B is the total drag of the two trucks divided by their drag in isolation as a function of the separation between the trucks. All of the curves should asymptote to unity at large values of separation. At small separations all of the four combinations experience a drag saving as one might anticipate. It is interesting - and unanticipated - that the degree of drag saving for the pairs of trucks depends upon which truck leads!

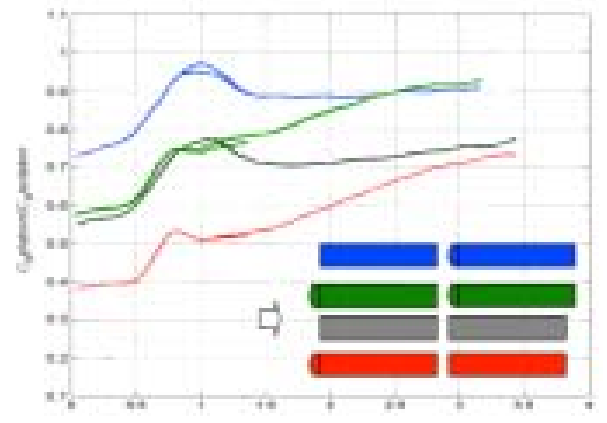

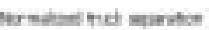

Fig. 9B. The average drag of the two-truck platoon for all four configurations. 


\section{References}

K. R. Cooper: The effect of front-edge rounding and rear-edge shaping on the aerodynamic drag of bluff vehicles in ground proximity. SAE paper No. 850288 (1985)

B. Nishri \& I. Wygnanski, "Effects of Periodic Excitation on Turbulent Flow Separation from a Flap," AIAA Journal, Vol. 36, No. 4, April 1998. 


\section{APPENDIX C}

\section{Continued Development and Improvement of Pneumatic Heavy Vehicles}

Principal Investigator: Robert J. Englar

Georgia Tech Research Institute (GTRI)

Atlanta, GA 30332-0844

(770) 528-3222; fax: (770) 528-7077;

e-mail: bob.englar@gtri.gatech.edu

Technology Development Manager: Sid Diamond (202) 586-8032, sid.diamond@ee.doe.gov

Technical Program Manager: Jules Routbort

(630) 252-5065,routbort@anl.gov

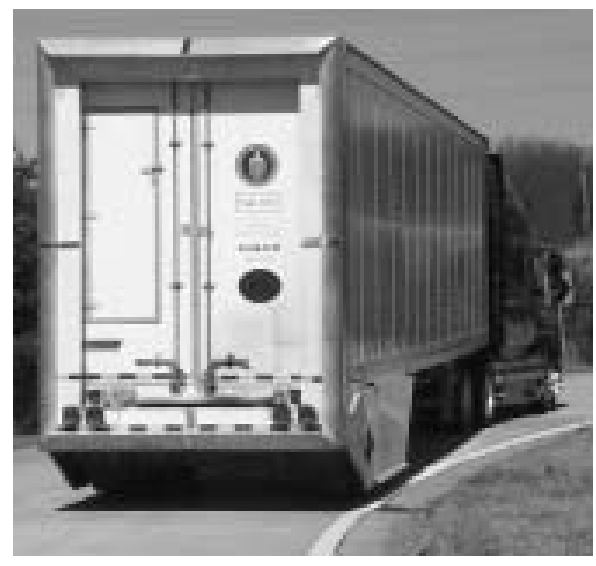

Contractor: Dept. of Energy - NNSA, NNSA Service Center, Oakland, CA,

Contract No.: DE-AC03-02EE50691

\section{Objective}

- Based on previous smaller-scale experimental evaluations at GTRI, which demonstrated up to $15 \%$ reduction in aerodynamic drag coefficient due to blowing and $10-12 \%$ due to the device's corner rounding for a combined drag reduction of $15-27 \%$, we are continuing the development of this pneumatic aerodynamic technology for parasitic energy loss reduction, fuel economy improvement, reduced emissions, and increased safety of operations for Heavy Vehicles (HV).

- Confirm these same benefits for Pneumatic Sports Utility Vehicles (PSUVs).

\section{Approach}

- Enhance the pneumatic aerodynamic capabilities of existing wind-tunnel and full-scale HV test models/vehicles, and then modify them to improve the drag-reduction properties exhibited during our Phase I of SAE Type-II fuel economy on-track testing.

- Identify pneumatic aerodynamic and geometry improvements to increase fuel economy by an additional factor of 2 to 4 over that exhibited in full-scale HV track tests.

- Conduct full-scale wind-tunnel development of Pneumatic SUVs with blowing applied to reduce drag; increase braking; increase traction; and improve stability and control.

\section{Accomplishments}

- New wind-tunnel tests have shown blowing produces drag reductions due to both aft flow separation elimination and base pressure recovery. New tests also demonstrated that active control can increase drag, showing that the device has the potential to help braking and safety of operation for both Pneumatic HVs and SUVs.

- Improvements needed for the Phase II full-scale PHV track test have been identified; new tunnel model is designed and being constructed to test in GTRI tunnel and confirm these before test vehicle modifications. 
- Design of full-scale PHV Phase II test vehicle based on these results is underway; indications from small-scale tunnel experiments are that a fuel economy of 7\% with blowing only are obtainable with these new blown configuration geometries, compared to a maximum (best case) of 5-6\% (not accounting for energy use for blowing) from Phase I road tests.

\section{Future Direction}

- Conduct Phase II of on-road full-scale HV testing and demonstrations of pneumatic aerodynamic drag-reduction, fuel-economy and safety of operation techniques to provide a confirming database allowing application of this technology to operational Heavy Vehicles and SUVs.

\section{Introduction}

Since aerodynamic drag is the major component of Heavy Vehicle (HV) resistance at highway speed and thus their related fuel economy, GTRI has been applying advanced aircraft aerodynamic technology using blowing to reduce that drag generation on generally-bluff high-drag vehicles. Using the pneumatic aerodynamic technology known as Circulation Control [Ref. 1] and certain trailer and gap geometry changes, we have been able to reduce drag coefficient $\left(\mathrm{C}_{\mathrm{D}}\right)$ on $\mathrm{HV}$ models by up to $15 \%$ reduction in aerodynamic drag coefficient due to blowing and $10-12 \%$ due to the device's corner rounding, for a total of $25-27 \%$ (see Fig. 3C and 6C) during a 4-year tunnel test program for DOE [2]. Of further advantage, we could also potentially be able to increase drag as needed for braking during downhill operation by rapidly blowing select trailing edge surfaces on the trailer without any moving parts, or could potentially also reduce the huge drag increase and loss of stability which occur when an HV experiences side winds or gusts. This multi-function potential of the blown configurations is seen in the wind tunnel data shown of Figure 1C.

Full-scale fuel economy tests were conducted [3] at the beginning of the current 2-year DOE program. Whereas preliminary Tuning Tests showed unofficial Fuel Economy Increase $(\% \mathrm{FEI})$ of over $15 \%$, the SAE Type-II official test-track results on a somewhat different Pneumatic HV (PHV) configuration showed maximum (best case) $\%$ FEI of only $5 \%-6 \%$, not accounting for energy use for blowing. The current program has thus concentrated on: determining the difference between wind-tunnel results and the less-than-expected full-scale performance; correcting the blown configuration; and preparing for a second fuel economy evaluation with the improved PHV vehicle. It is noted here that the present DOE program does not correspond directly to FY03. Funding for year-long Phase $\mathrm{V}$ discussed below started 7/14/2002, and Phase VI (FY 03 funding) was not initiated until May 29, 2003. The recent results of both Phases are presented as FY03 results for PHVs and for Pneumatic Sports Utility Vehicles in the following sections because they occurred in FY03. 


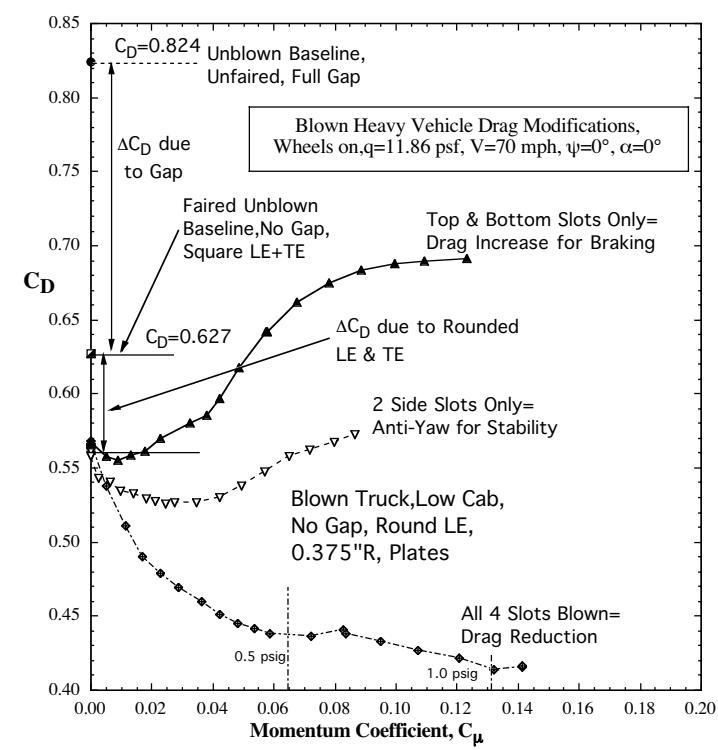

Fig. 1C- Drag reduction or drag increase demonstrated by GTRI model PHV, depending on blowing location

\section{Experimental Details and Results}

Experimental wind-tunnel developments of this technology conducted on smallerscale PHV model under previous DOE funding [Refs. 2, 3, 4] led to two fullscale Tuning Tests conducted at Volvo Truck's facilities, plus an SAE Type-II Fuel Economy Test conducted at the 7.5mile test track at Transportation Research Center in Ohio, with the results reported above. Since this drag reduction and the fuel economy increase were less on the test PHV vehicle than the tunnel data predicted, we returned to the tunnel to determine the reasons. The generic tunnel model was modified so that it more closely resembled the "more-draggy" full-scale test article, and then re-tested. Then, implicated components from the track-test vehicle were corrected and the model re-tested each time. Figure 2C shows how correction of each component on the track-test $\mathrm{PHV}$ reduced the $\mathrm{C}_{\mathrm{D}}$ below that of the unblown Baseline reference truck (Run 36). Run 239's configuration is most like our TRC blown test truck. Figure $3 \mathrm{C}$ shows the drag reductions that would occur for this test truck if these less-than-optimum geometries were corrected, and then Figure 4C shows the increases in fuel economy projected for highway speeds (where drag is half or more of total resistance) relative to the Baseline truck if these items were revised. Results

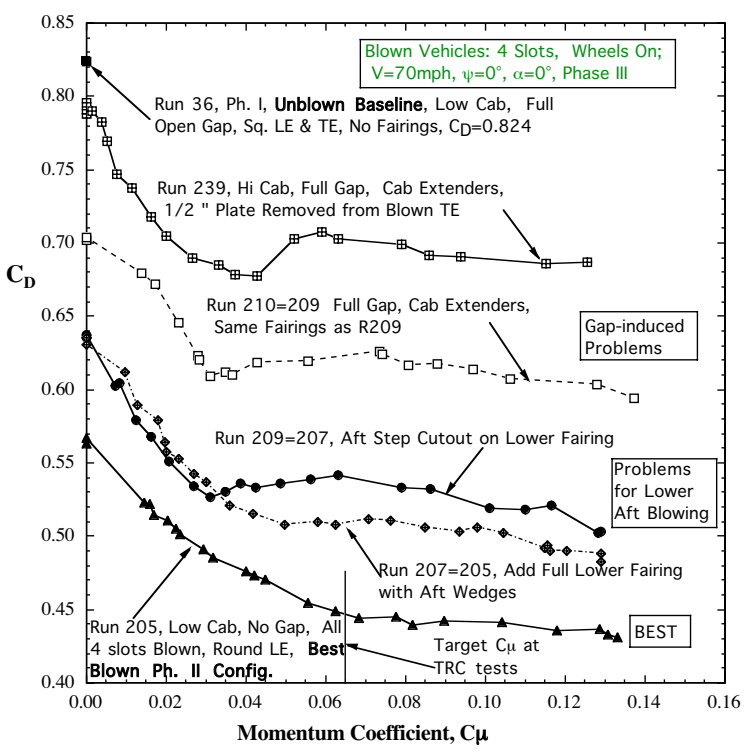

Fig. 2C- Tunnel results: drag changes with model correction and also with blowing variation

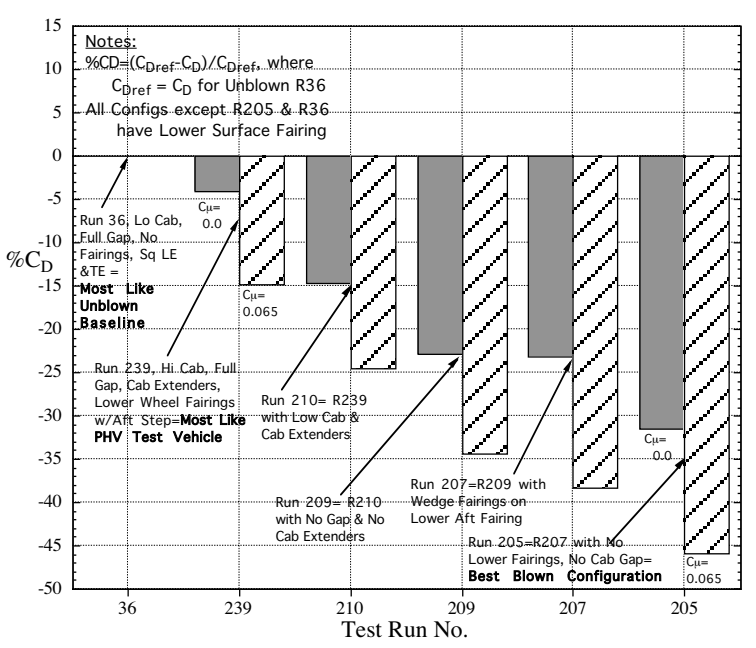

Fig. 3C- Incremental drag changes relative to the unblown baseline configuration of Figure 2 


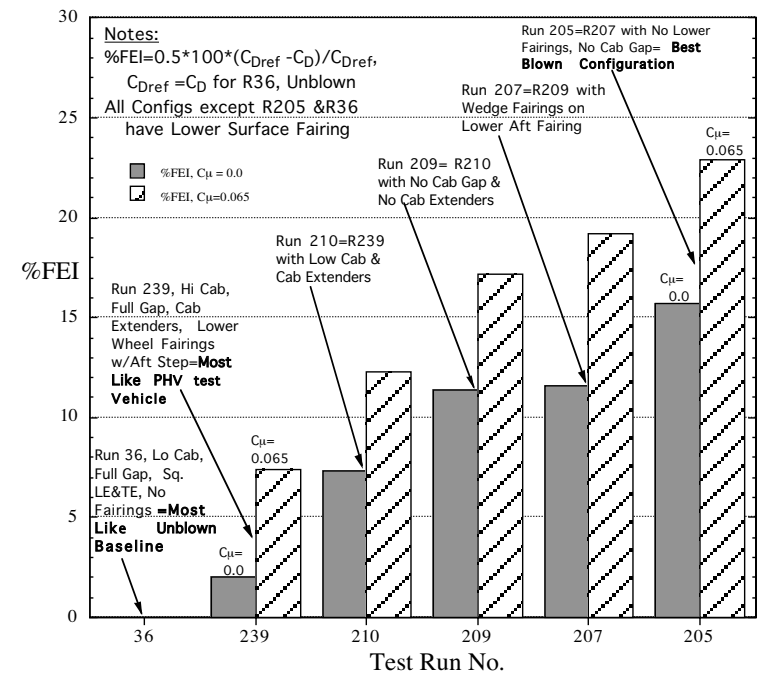

Fig. 4C- Resulting fuel efficiency increase with configuration and blowing variations

show fuel economy increases (\%FEI) of up to $16 \%$ for the unblown PHV geometry, and up to $23 \%$ for the blown vehicle at test $\mathrm{C} \mu$. This indicates a 7\% FEI for blowing without considering geometry modifications which provides a slightly better result than the recent ontrack results of 5-6\% FEI, and thus these data provide a guide towards redesign of the test vehicle for an improved lowdrag PHV truck.

An important finding in this data is the effect of the gap between the tractor and trailer. Figure 5C shows our PHV test vehicle, which even with its cab extenders, still has an obvious gap. Note in Figure 2C, 3C, and 4C the adverse effect of a gap discontinuity on the small scale model. An appropriate gap side plate was developed. Tunnel test results, Figure 6, show this simple but feasible device to be nearly as effective in eliminating the gap disruption as a nonfeasible solid gap ("NO Gap"). Note the continued drag reduction occurring with additional leading-edge (LE) blowing incorporated with these gap plates.

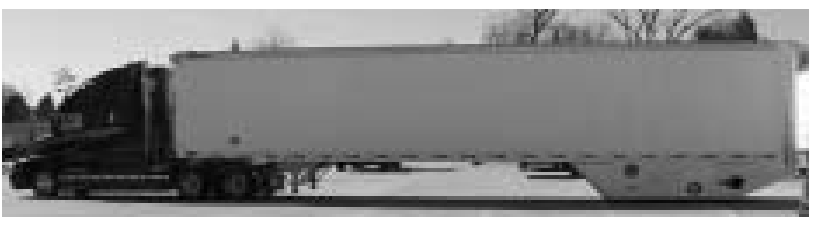

Fig. 5C- PHV test vehicle with gap between tractor and the trailer

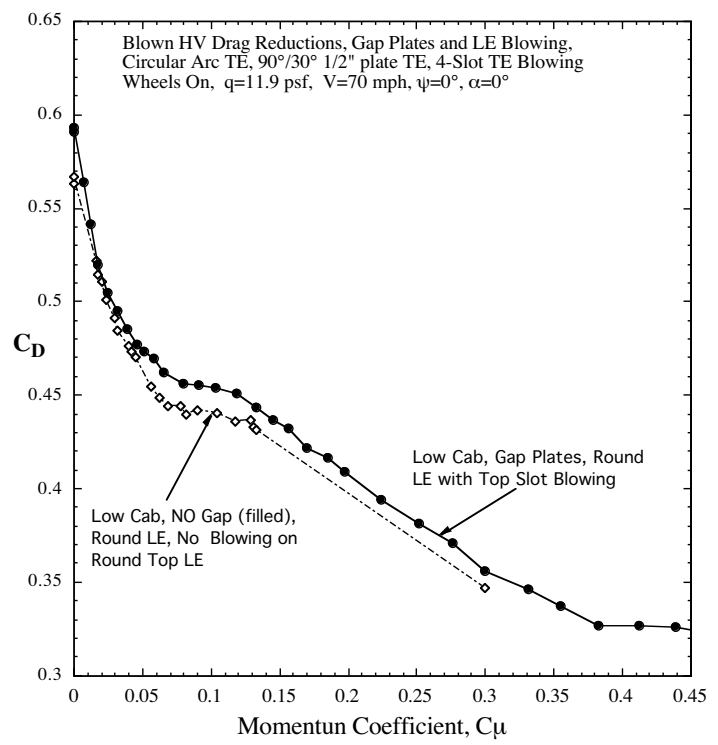

Fig. 6C- A practical solution for gap separation: Gap Side Plate

In addition to drag reduction due to elimination of aft flow separation, two other improvements were shown during these recent tunnel tests. Blowing on the aft trailer corners converted the suction on the aft doors (a drag component) into overpressure there (a drag reduction $=\mathrm{a}$ "push"). Figure 7C confirms this $(+\mathrm{Cp}$ is pressure), as does integration of the rear door pressure force to yield negative incremental drag components produced by blowing. Up until the higher values of blowing coefficient $\mathrm{C} \mu$, the incremental $C_{D}$ values due to the blowing overpressures are greater than the total $\mathrm{C} \mu$ expended. Additional $\Delta \mathrm{C}_{\mathrm{D}}$ still 
missing from these blowing gains are drag reductions due to flow-separation prevention (not shown here).
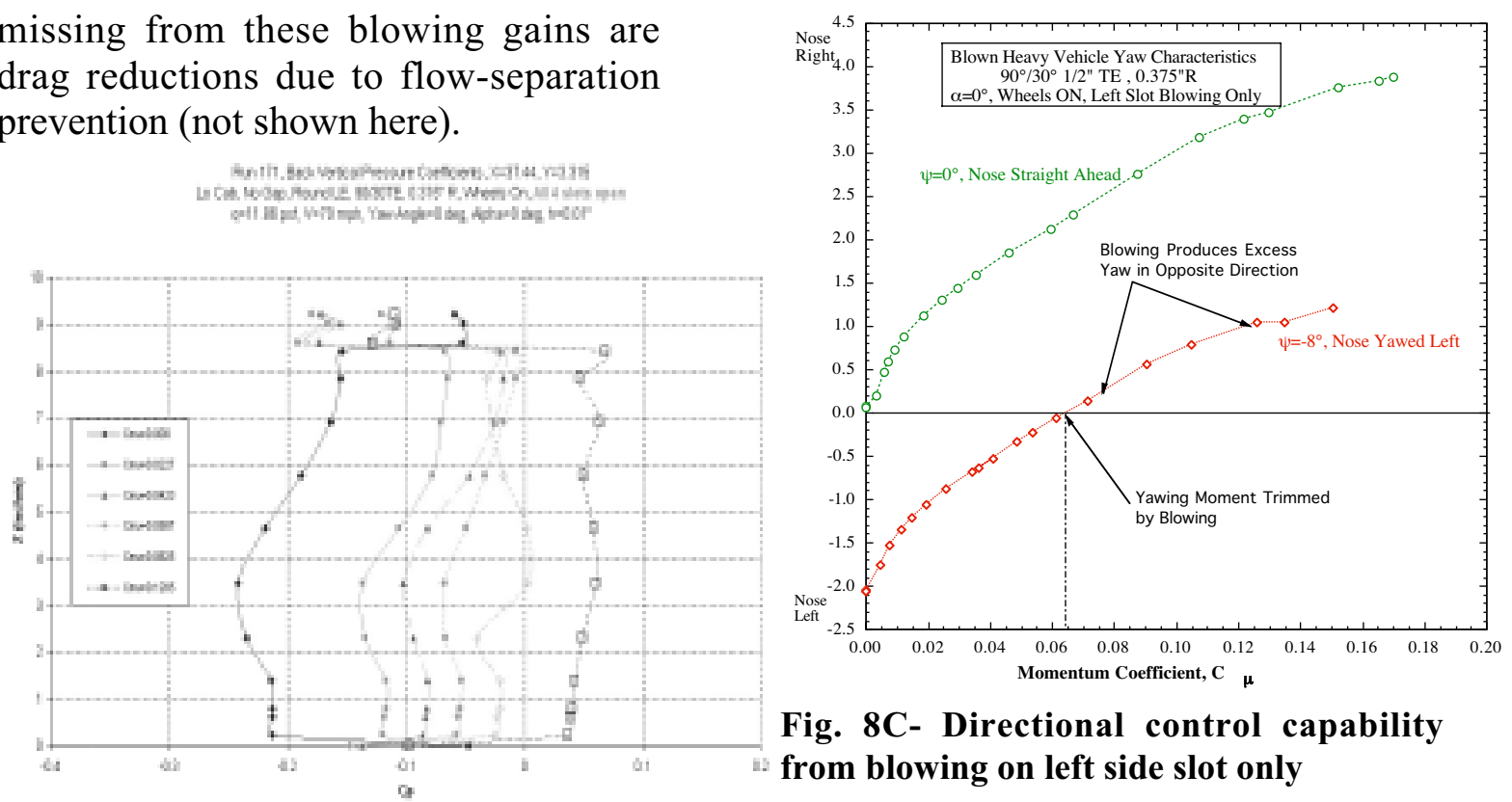

Fig. 8C- Directional control capability from blowing on left side slot only

Fig. 7C- Static pressure recovery on back doors due to blowing

\begin{tabular}{|c|c|}
\hline $\mathrm{C} \mu$ & $\Delta \mathrm{C}_{\mathrm{D}}$ from Base Pressure \\
\hline \hline 0.00 & 0.000 \\
\hline 0.023 & -0.034 \\
\hline 0.042 & -0.052 \\
\hline 0.059 & -0.060 \\
\hline 0.083 & -0.075 \\
\hline 0.121 & -0.114 \\
\hline
\end{tabular}

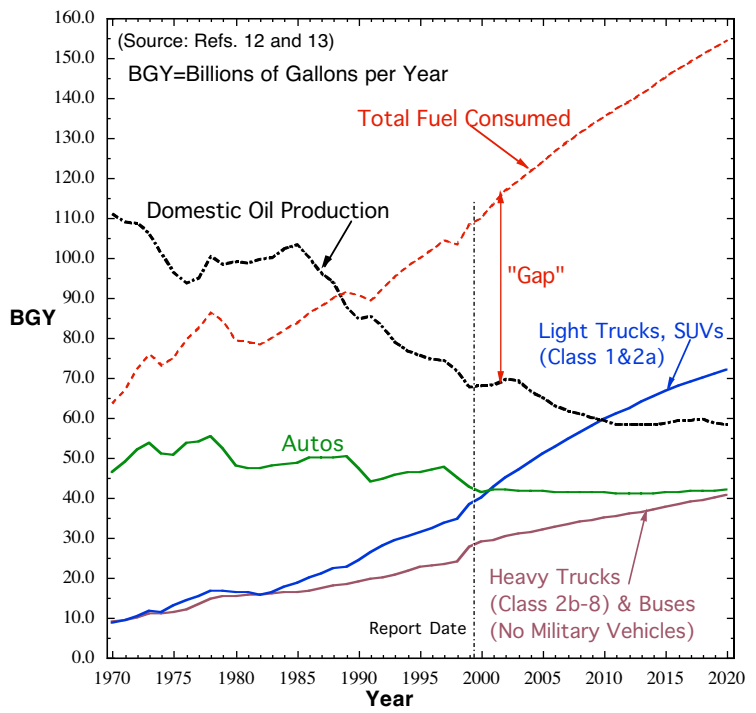

Blowing on only one vertical slot of the trailer has been shown experimentally to dramatically reduce drag experienced during a side wind (yaw), it also could produce aft side force to counteract destabilizing yawing moment, and thus possibly yield directional control. Figure 8C shows how the unstable yawing moment, $\mathrm{C}_{\mathrm{N}}$, at $\mathrm{C} \mu=0$ during nose-left yaw of the small-scale model can be reduced to zero by blowing the aft left side slot, and then a stabilizing opposite moment can be generated by slightly increasing left-slot blowing.

Fig. 9C- Highway energy usage comparisons by vehicle type (from DOE, Ref. 5)

Realizing that drag reduction may be even more important for Sports Utility Vehicles (SUVs) than HVs (see Figure 9C DOE fuel usage data, from Ref. 5), we conducted full-scale wind tunnel development of this pneumatic technology applied to a GM Suburban SUV, Figure 10C. With a variety of blowing configurations tested, we 
showed in Figure $11 \mathrm{C}$ that blowing could either reduce drag for fuel efficiency or could increase drag for braking. We also confirmed again that blowing only one side slot could effect drag and yaw during high side-wind angles, as Figure $12 \mathrm{C}$ shows. The restoration of directional stability using no moving surfaces is thus verified.

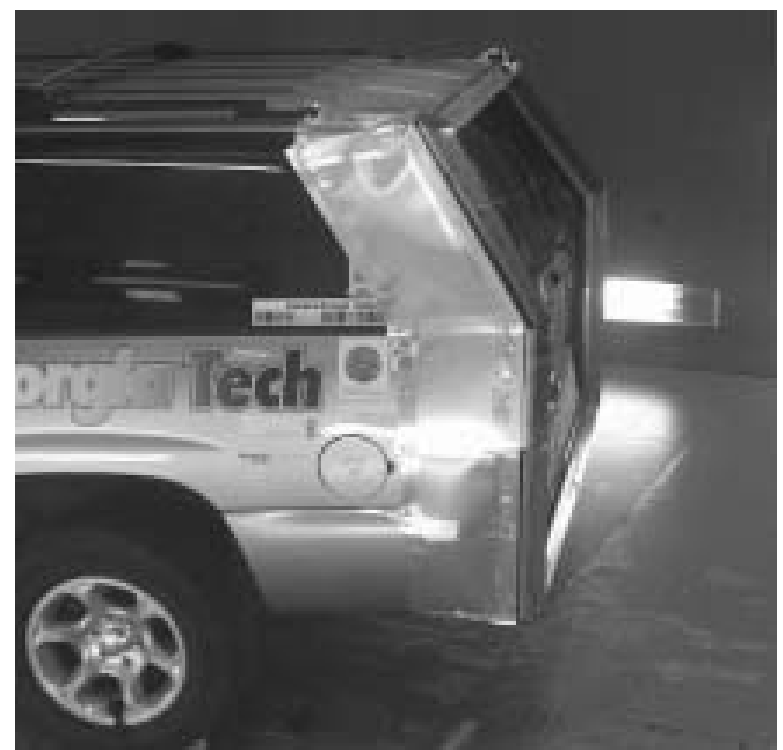

Fig. 10C- Tufts showing blowing and jet turning on PSUV in Lockheed wind tunnel

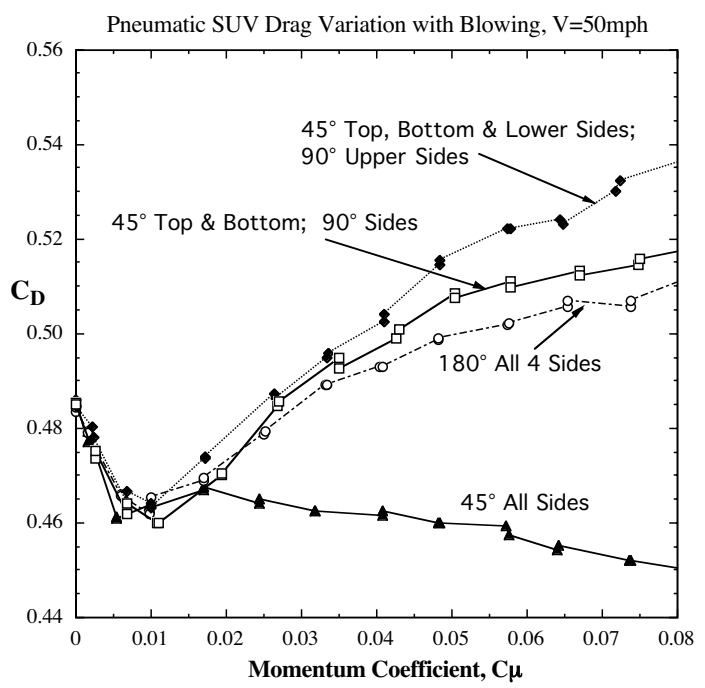

Fig. 11C- PSUV drag coefficient changes due to blowing on various configurations

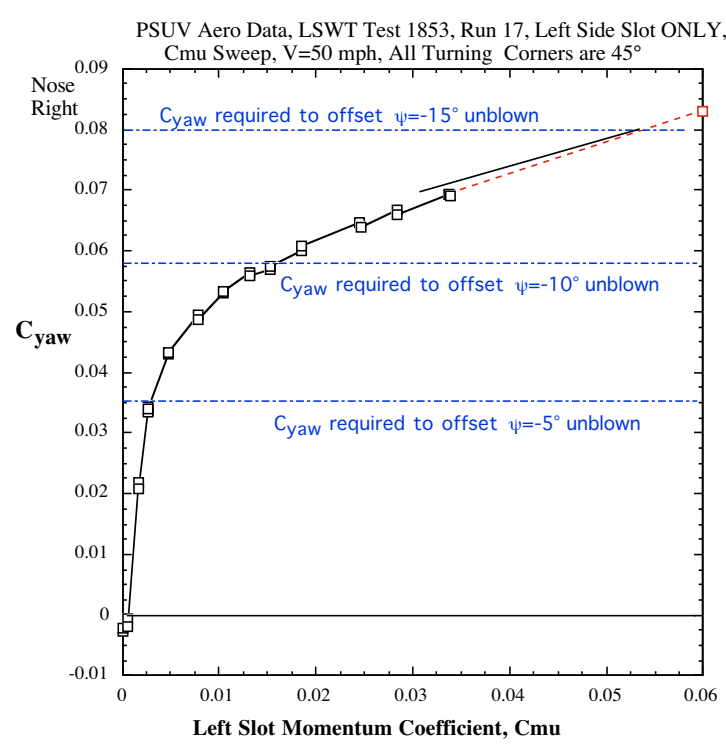

Fig. 12C- PSUV drag coefficient changes due to blowing on various configurations

\section{Discussion}

The above wind-tunnel tests to identify reasons for the less-than-expected drag reduction on the full-scale PHV road test vehicle have determined a number of configuration improvements needed in the blowing geometries, the trailer under-side fairings and the tractor-trailer gap. These, when corrected, should yield the earlier-recorded small-scale model drag reductions of up to $15 \%$ reduction in aerodynamic drag coefficient due to blowing and $10-12 \%$ due to the device's corner rounding, for a total of 25$27 \%$. We have incorporated these into the design of a next-generation smallerscale PHV tunnel model which now incorporates a generic current-day tractor geometry, Figure 13C. This new model (CAD details of which are available but drawings are too large to include herein) includes new blown trailing-edge geometries to yield higher base pressure recovery, no lower surface fairings, and other more realistic under-trailer and wheel geometries. A 
new mounting strut is now from the top of the model so that interaction between the wheels, lower surface geometries and the road can be properly simulated. We will test this new PHV configuration in the GTRI Model Test Facility tunnel for comparison to the previous model performance and to estimate new fullscale PHV performance. This data will include many of the real vehicle protrusions (such as struts, differentials, floor spars, etc.) that could easily be detrimental to full-scale performance and fuel economy. Test results are to be incorporated into modification of the real test vehicle (see page 1 and Figure 5C) into the best performing blown configuration in preparation for a Phase II SAE fuel economy test program for the PHV and baseline trailer in FY04.

The full-scale wind tunnel results for the Pneumatic SUV confirmed both drag reduction, yaw control, stability restoration and braking increase. These full-scale aerodynamic stability and control results are virtually impossible to determine during road tests, so these PSUV tests provide invaluable data for stability \& control, and safety for SUVs. We realize that we have not yet optimized the blowing geometries on the contoured back surfaces of the SUV (far more complex than the square-edged HV trailer aft end) and that future work is needed here. However, as Figure 9C verifies, the sheer number of SUVs and pickup trucks on the American roads make this drag-reduction effort quite important, as does the promise of improved stability and braking for vehicles frequently in need of both.

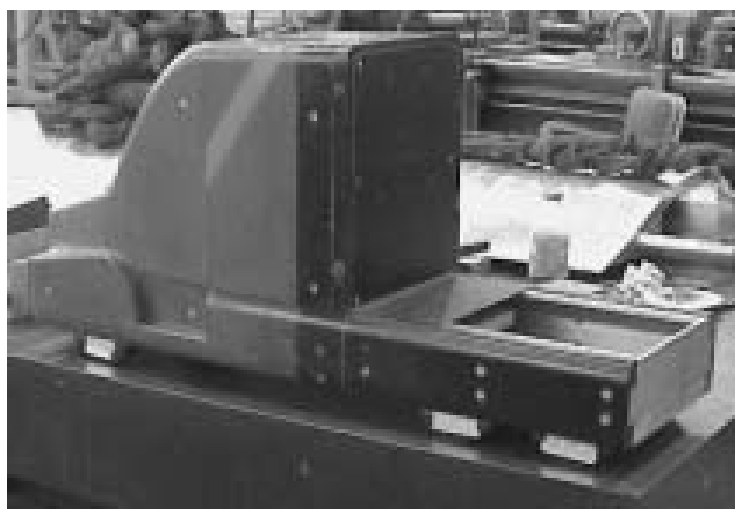

Fig. 13C- Generic Conventional Model (GCM) tractor to be included with new GTRI PHV test model

\section{Conclusions}

To advance the state of development of pneumatic aerodynamics for improvement of $\mathrm{HV}$ and SUV drag reduction, fuel economy, braking, stability and safety of operation, GTRI and its team members have continued in FY03 our previous program for DOE. We have conducted investigations for both model-scale wind tunnel and fullscale test track HVs and full-scale tunnel investigations on an SUV of these advanced capabilities, as well as to identify and correct aerodynamic problems areas on our first fueleconomy test on a full-scale Pneumatic Heavy Vehicle. Results of this recent effort include:

- We have identified the aero problem areas of our first PHV road test and how to correct these; current indications are that drag reductions of $14 \%$ and fuel economy increases of $7 \%$ are projected to result for these new configurations at highway speeds due to blowing alone.

- A new model test configuration based on current generic tractor geometry and the above results has been designed, the upcoming tunnel testing at GTRI of which will provide the guidance for 
modification of the full-scale PHV test vehicle.

- Full-scale tunnel testing of pneumatic aerodynamics on an SUV has verified pneumatic drag reduction, directional stability, and the versatility of these blown no-moving-part, performanceenhancing, economy-increasing, and stability devices to improve the operation of SUVs. These results now lead to re-design and modification of the PHV road-test vehicle in preparation for the Phase II SAE Type-II fuel economy test scheduled for FY04.

\section{References}

1. Englar, Robert J., "Circulation Control Pneumatic Aerodynamics: Blown Force and Moment Augmentation and Modification; Past, Present and Future," AIAA Paper 2000-2541, Denver, CO, June 19-22, 2000.
2. Englar, Robert J., "Advanced Aerodynamic Devices to Improve the Performance, Economics, Handling and Safety of Heavy Vehicles," SAE Paper 2001-01-2072, Washington, DC, May 1416, 2001.

3. Englar, R. J. "Pneumatic Heavy Vehicle Aerodynamic Drag Reduction, Safety Enhancement, and Performance Improvement," in the Proceedings of the UEF Conference on "The Aerodynamics of HeavyVehicles: Trucks, Buses, and Trains", Monterey-Pacific-Grove, CA, Dec. 2-6, 2002.

4. Englar, Robert J. "Drag Reduction, Safety Enhancement and Performance Improvement for Heavy Vehicles and SUVs Using Advanced Pneumatic Aerodynamic Technology", SAE Paper 2003-01-3378, accepted and to be presented November 1012, 2003.

5. "EIA Annual Energy Outlook 2000," DOE/EIA-0383 (2000), December, 1999 and "AEO 2001". 


\title{
APPENDIX D
}

\section{Computational and Experimental Investigation of Drag Reducing Add-on Devices and the Flow Field around a Simplified Tractor-Trailer Geometry}

\author{
Principal Investigator: $K$. Salari \\ Co-Investigators: J. Ortega, R. McCallen \\ Lawrence Livermore National Laboratory \\ P.O. Box 808, Livermore, CA 94551-0808 \\ (925) 423-0958; mccallen1@,llnl.gov
}

Technology Development Manager: Sid Diamond

202-586-8032, sid.diamond@,ee.doe.gov

Technical Program Manager: Jules Routbort

630-252-5065, routbort@anl.gov

Contractor: Lawrence Livermore National Laboratory

Contract No.: W-7405-ENG-48

\section{Objective}

- Increase the fuel economy of class 8 tractor-trailers by reducing the aerodynamic drag.

- Identify and to understand the key flow structures around the vehicle that significantly impact the aerodynamic drag.

- Investigate the capability of modern computational tools to predict the flow field around a tractortrailer type geometry, in the wake of the trailer, in the underbody, and in the gap between the tractor and the trailer.

- Design new add-on devices that significantly reduce aerodynamic drag for class 8 tractor-trailers achieved by small-scale wind tunnel tests supported by computational modeling and simulation.

- Present a list of effective drag-reducing add-on devices to the OEM's for further full-scale testing.

\section{Approach}

- LLNL is developing and utilizing state-of-the-art numerical algorithms and advanced turbulence models to create computational tools that can accurately predict the complex flow field around modern tractor-trailer type geometries. Significant emphasis is placed on validation calculation to ensure proper evaluation of the computational results compared to experimental data.

- Investigate key flow structures, such as trailer wake, the gap flow between the tractor and the trailer, and underbody, which significantly impact the aerodynamic drag of heavy vehicles. This is accomplished through use of careful discovery and validation experiments and computational modeling and simulation of the complex flow field around these vehicles. 
- Three truck-like geometries, GTS, USC modified GTS, and GCM, are used to investigate the predictive behavior of various numerical approaches and turbulence models. These models represent simple to modern tractor-trailer geometries, with the GCM representing a realistic/modern model.

- Discovery experiments at NASA Ames's small-scale 3'x4' wind tunnel provide a mechanism to study the effectiveness of LLNL proposed drag reducing add-on devices.

- Given the knowledge obtained from the discovery experiment and computational simulations for the flow structures in the wake of the trailer, underbody, and the gap, new add-on devices can be designed to reduce aerodynamic drag of the class 8 tractor-trailer.

\section{Accomplishments}

- The steady and unsteady turbulent flow fields around a tractor-trailer geometry have been successfully simulated with emphasis on the wake of the trailer, underbody, and the gap flow structure

- LLNL completed simulations at $0^{\circ}$ and $10^{\circ}$ yaw for GTS geometry with three different turbulence models for a total of nine runs. The predicted result for each turbulence model is compared to the experimental data of NASA Ames's 7'x10' wind tunnel. A detailed study was conducted to investigate the sensitivity of the solution to grid resolution around sharp corners and in the vicinity of the separated region.

- Completed unsteady gap flow simulation for USC modified GTS model using a coarse mesh (6.2 million elements). Unsteady results are compared to USC experimental data.

- The LLNL add-on device for the gap was successfully simulated with the USC modified GTS geometry. The new add-on device stabilizes the unsteady flow in the gap and, in turn, potentially reduces the aerodynamic drag.

- New LLNL drag reducing add-on devices have been designed based on the ascertained knowledge of the flow field from the experimental investigation and computational simulations. Five records of invention for new LLNL add-on devices have been filed.

- Experimental discovery is underway at NASA Ames to determine the effectiveness of the proposed new add-on devices and to investigate the complex flow field around the vehicle.

\section{Future Direction}

- Continue with development of the predictive modeling capability through use of the state-of-theart turbulence modeling and numerical algorithms to simulate the complex flow field around the class 8 tractor-trailer.

- A new hybrid RANS/LES turbulence model is under development that could improve the accuracy of the flow field prediction around the heavy vehicle.

- Continue with testing the proposed LLNL add-on devices to reduce the aerodynamic drag of heavy vehicles through the discovery experiments.

- Present a list of effective add-on devices to OEM's for further full-scale testing.

- Investigate the aerodynamics of wheels and wheel wells for brake cooling.

- Investigate the aerodynamics of coal rail cars.

- Investigate the aerodynamics of underbody/wheel splash and spray. 


\section{Summary}

The LLNL activities for FY03 include computational modeling and simulation, design of drag reducing add-on devices, and performing discovery experiments at the NASA Ames facility. The LLNL goal for the computational effort is to provide guidance on drag reduction strategies that could be implemented with add-on devices. This is accomplished by utilizing the computational results to provide insight into the flow structures that have been identified as key to reducing the aerodynamic drag of heavy vehicles. The focus of the design of add-on devices is to improve or stabilize the turbulent unsteady flow field in the gap, the wake, and the underbody of the vehicle to reduce the aerodynamic drag. The focus of the experimental effort is to provide detailed information on various LLNL proposed drag reduction concepts. There are three geometries under investigation, GTS, USC modified GTS, and GCM (Fig 1D). These geometries represent simple to modern tractor-trailer models with GCM representing a realistic/modern tractor-trailer geometry.

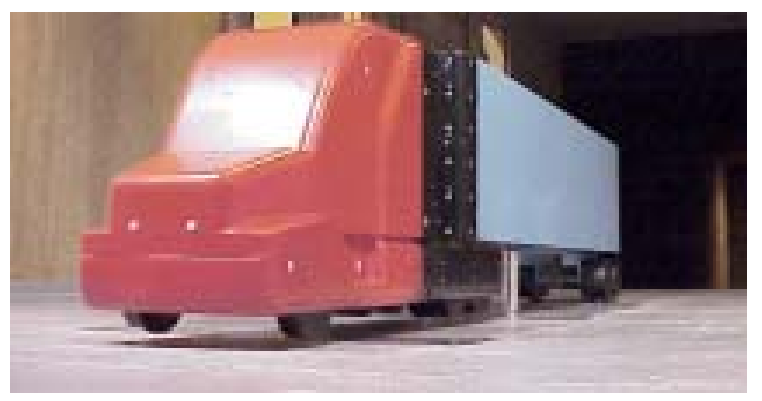

Fig 1D - Generic Conventional Model (GCM)

Significant emphasis is placed on validation calculation to ensure proper evaluation of the computational results compared to experimental data. The validation calculation requires modeling a section of the NASA Ames's 7'x10' wind tunnel and properly matching the flow condition in the test section of the tunnel to the experimental data. The boundary layer profile and static pressure at the wind tunnel test section are typically used to properly set the inflow and outflow to the computational domain. The advantage of this approach is to use the raw or unmodified experimental data to validate the computational results. This is a requirement for performing the validation calculation.

Steady and unsteady RANS simulations are performed to model the complex flow field around the vehicle, in the gap between the tractor and the trailer, and the trailer wake. The experimental data of USC and NASA are used to benchmark and validate the computational results. The small-scale 3' $x 4$ ' wind tunnel at NASA Ames is providing an inexpensive and flexible way to fully investigate the selected drag reduction devices using the USC modified GTS model. The goal for the experimental investigation is to identify one or two add-on devices that clearly standout from other known and effective drag reducing devices.

\section{Computational Simulations}

Flow simulation of the GTS model at $0^{\circ}$ and $10^{\circ}$ yaw in the NASA Ames's 7'x10' wind tunnel and the modified GTS with the non-dimensional gap distance of 0.72 at $0^{\circ}$ yaw in the USC tunnel are completed. Steady and unsteady RANS flow simulations are performed with NASA's Overflow code that uses overset grids. 
For all GTS simulations in the NASA wind tunnel, the flow condition is obtained from the available experimental data, run 7 point 9. The following flow conditions were provided for this run: Mach number 0.28, Reynolds number $2.08 \times 10^{6}$ (based on trailer width), total pressure $102649.2 \mathrm{~N} / \mathrm{m}^{2}$, total temperature $284.5^{\circ} \mathrm{K}$, static pressure $97339.1 \mathrm{~N} / \mathrm{m}^{2}$, static temperature 280.2 ${ }^{\circ} \mathrm{K}$, and air density $1.206 \mathrm{~kg} / \mathrm{m}^{3}$.

The following simulations have been completed for GTS geometry:

- $0^{\circ}$ and $10^{\circ}$ yaw

- Three turbulence models, 1-equation model of Spalart-Allmaras (SA), two-equation models of Wilcox $k-\omega$ and Menter SST.

- Two-grid sizes of 3.7 and 12.2 million elements.

Figure 2D shows a complex flow field with multiple vortical structures and a separation bubble at the leeward side of the vehicle highlighted by particle traces around the GTS geometry at $10^{\circ}$ yaw in the NASA Ames's 7'x10' wind tunnel.

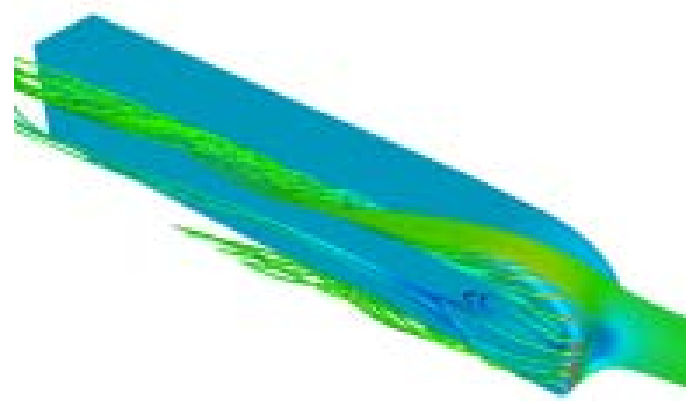

Fig 2D- Particle traces are colored by velocity magnitude, $10^{\circ}$ yaw, and GTS model
Table 1D shows the computed drag coefficient and the experimentally measured drag values. The subscript $\mathrm{W}$ refers to the static pressure measured on the test-section tunnel wall and the subscript $\mathrm{R}$ refers to the static pressure measured upstream of the test section (see [1]). The drag coefficients obtained from the simulations are based on the dynamic pressure calculated using the test section dynamic pressure. All turbulence models have similar prediction for viscous drag; however, the SA and SST models significantly overpredict the pressure drag. Similarly, the Wilcox $k-\omega$ model over predicts the pressure drag, but provides a drag coefficient closest to the experimental value. All tested RANS turbulence models have difficulty with predicting the correct pressure drag. Since the trailer wake has a significant influence on the pressure drag, this suggests that RANS turbulence models could have difficulty predicting the correct flow structure in the wake of the trailer. Previous simulations on the GTS geometry using the SA model are presented in [2]. This work includes careful investigation of the influence of grid resolution and inflow and outflow boundary conditions on the predictive capability of the SA model. Similar results are reported on the aerodynamic forces. 


\begin{tabular}{|c|c|c|c|}
\hline Drag & Viscous & Pressure & Total \\
\hline$k-\omega, \mathrm{cg}$ & 0.103 & 0.188 & 0.290 \\
\hline$k-\omega, \mathrm{mg}$ & 0.101 & 0.176 & 0.277 \\
\hline SST, cg & 0.091 & 0.273 & 0.364 \\
\hline SST,mg & 0.092 & 0.258 & 0.350 \\
\hline SA, mg & 0.096 & 0.294 & 0.390 \\
\hline \multicolumn{2}{|c|}{ NASA Experiment, $\mathrm{C}_{\mathrm{D}, \mathrm{W}}$} & 0.249 \\
\hline \multicolumn{3}{|c|}{ NASA Experiment, $\mathrm{C}_{\mathrm{D}, \mathrm{R}}$} & 0.263 \\
\hline
\end{tabular}

Table 1D - Computed aerodynamic forces,

GTS model, $0^{\circ}$ yaw, coarse grid (cg), and medium grid (mg)

Figure 3D shows a velocity vector field in the wake of the GTS trailer at the symmetry plane with SST turbulence model. Figure 4D shows the timeaveraged PIV data from NASA Ames at the same cut plane as that in Figure 3D. In the experimental data, there is a dominant recirculation zone at the lower base of the trailer. On the other hand, there are two similarly sized recirculation zones on the lower and the upper part of the base of the trailer in the computational result. Noticeably, the flow structures in the computational result do not match that of the experimental PIV data and, hence, this difference may explain the disagreement in the predicted drag. The Wilcox $k-\omega$ model has a similar wake prediction as that of the SST model; however, the SA model predicts a much smaller wake size with two symmetric recirculation zones at the lower and the upper part of the base of the trailer.

The RANS models used in this study did a reasonable job in predicting the pressure and skin friction distributions

on the surface of the GTS. However, if absolute drag and predicting the wake flow structure is of interest, other turbulence more sophisticated turbulence models should be investigated.

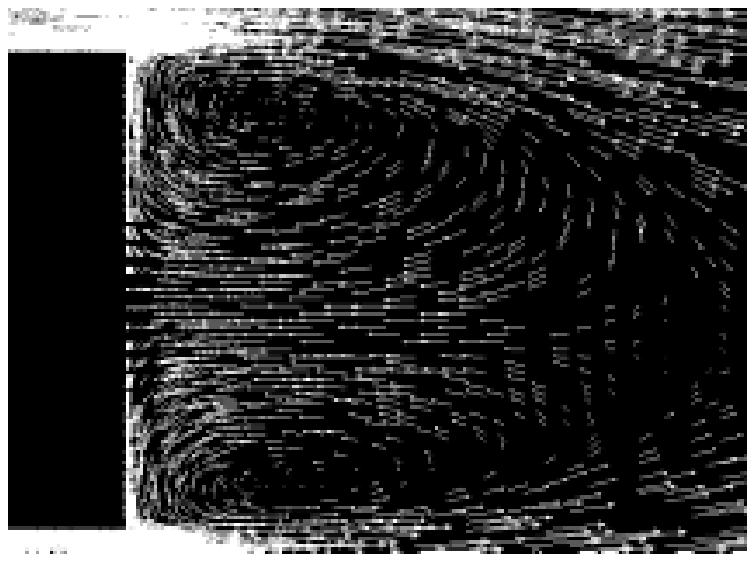

Fig 3D - Wake flow structure, SST model, $0^{\circ}$ yaw, symmetry plane, GTS model

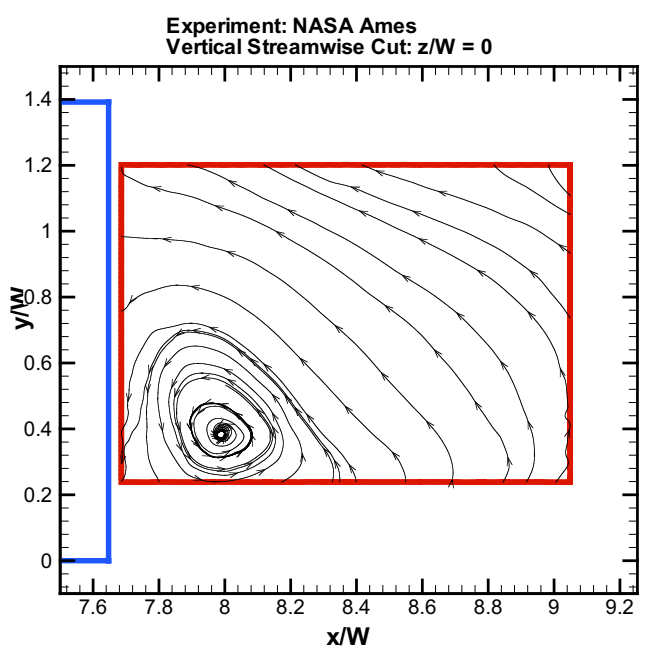

Fig 4D- Wake flow structure, NASA time averaged PIV data, $0^{\circ}$ yaw, symmetry plane, GTS model

Table 2D shows the computed lift, drag, and side force coefficients for the $10^{\circ}$ yaw case and the experimentally measured values. The Wilcox $k-\omega$ and the Menter SST model have problems in 
predicting the lift coefficient. Similarly, the predicated drag coefficient is off significantly. However, both turbulence models have a better prediction for the side force. Note that there exists a significant flow separation (Fig. 5) at the leeward side of the GTS model. This type of flow separation poses a challenge for the RANS turbulence model.

\begin{tabular}{|c|c|c|c|}
\hline & Lift & Drag & Side \\
\hline$k-\omega, \mathrm{mg}$ & -0.004 & 0.581 & 1.127 \\
\hline SST, cg & 0.006 & 0.651 & 1.129 \\
\hline SST, mg & -0.010 & 0.664 & 1.137 \\
\hline Exp, $\mathrm{C}_{\mathrm{D}, \mathrm{W}}$ & 0.021 & 0.292 & 1.253 \\
\hline Exp, $\mathrm{C}_{\mathrm{D}, \mathrm{R}}$ & 0.022 & 0.312 & 1.338 \\
\hline
\end{tabular}

Table 2D - Computed aerodynamic forces,

GTS model, $10^{\circ}$ yaw, coarse grid (cg), and medium grid (mg)

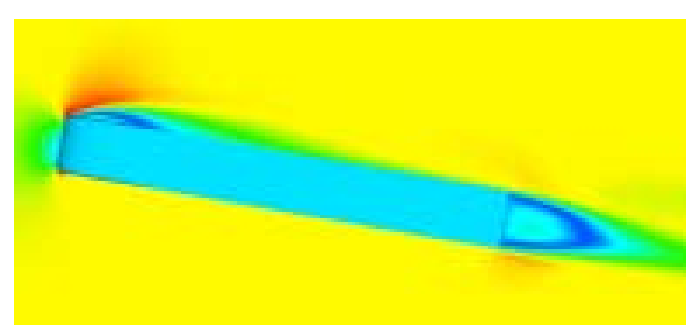

Fig 5D $-10^{\circ}$ yaw, velocity magnitude contours, half height cut plane, SST model,

GTS geometry

Figure 6D shows the vortex cores that are present in this complex flow field. The presence of multiple vortex cores on the top surface of the GTS geometry, suggest multiple separation and reattachment lines. Similarly, a dominant vortex is present on the leeward side of the GTS that extends the length of the trailer. The position of these separation and reattachment lines can be compared to that of the NASA experimental data.
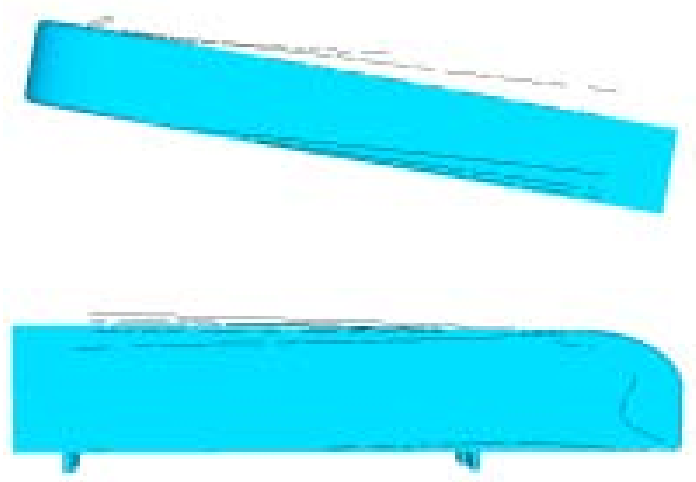

Fig 6D - vortex core positions, $10^{\circ}$ yaw, SST model, GTS geometry

Unsteady RANS simulations are conducted on the USC modified GTS geometry with a normalized gap distance of 0.72 , which is above the critical gap distance of 0.5 . At this gap distance, the flow experiences unsteadiness and could have multiple stable modes that are either symmetric or asymmetric. Also, the drag contribution from the gap flow significantly increases due to this unsteady behavior. Low drag for the gap relates to stable symmetric flow and high drag relates to the unsteady asymmetric flow. Figure 7D presents a snapshot of the unsteady flow field around the modified GTS model highlighted by particle traces colored by velocity magnitude. This figure shows the external flow reaching into the gap and exiting on the side. This is the typical unsteady asymmetric flow representing a high drag situation.

The gap flow also significantly interacts with the external flow and the wake of the trailer as shown in Figures 8D and 9D. This suggests that previous 
investigations [3], which isolated the base of the trailer for reasons of reducing the size of the computational domain, may not properly capture the unsteady turbulent flow in the wake.

The unsteady RANS simulation conducted with Overflow is capturing the symmetric and asymmetric flow field in the gap as shown in Figure 10D. This figure compares the computed results with the USC experimental data. A simple splitter plate was introduced in the gap and shown in Figures 11D and $12 \mathrm{D}$ to stabilize the gap flow. This device will be further investigated by USC as a possible drag reducing add-on device for the gap.

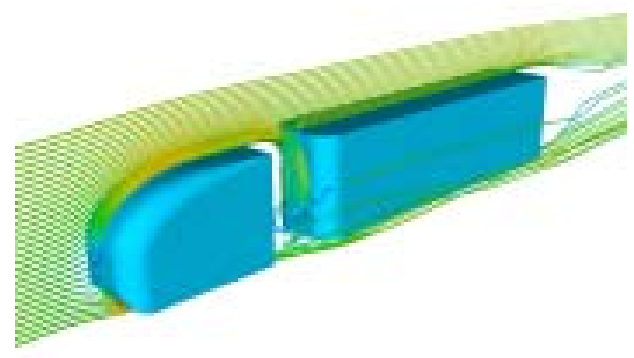

Fig 7D - Particle traces colored by velocity magnitude, $0^{\circ}$ yaw, $k$ - $\omega$ model, modified GTS geometry with 0.72 gap size

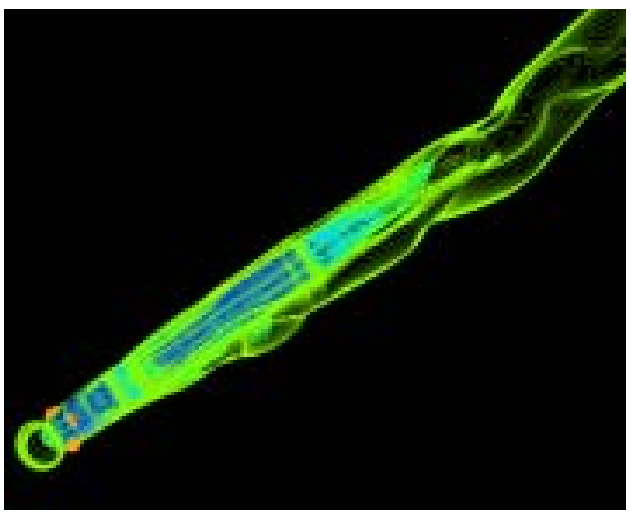

Fig 8D -velocity magnitude contours, $0^{\circ}$ yaw, $k-\omega$ model, modified GTS geometry with 0.72 gap size

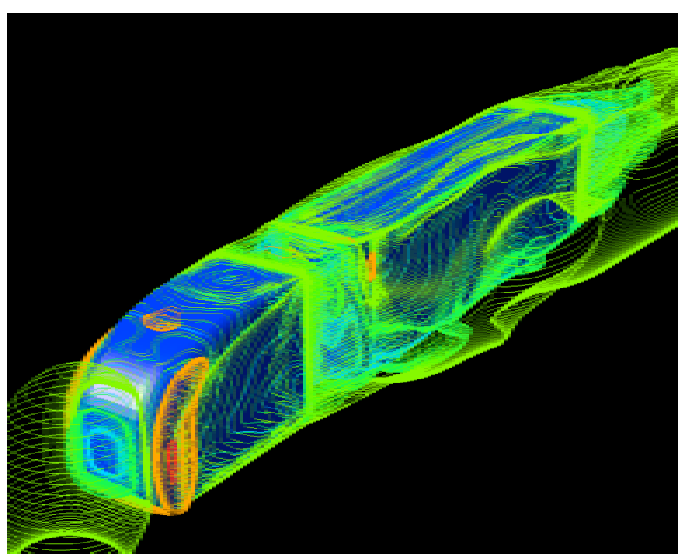

Fig 9D -velocity magnitude contours, $0^{\circ}$ yaw, $k$ - $\omega$ model, modified GTS geometry with 0.72 gap size 

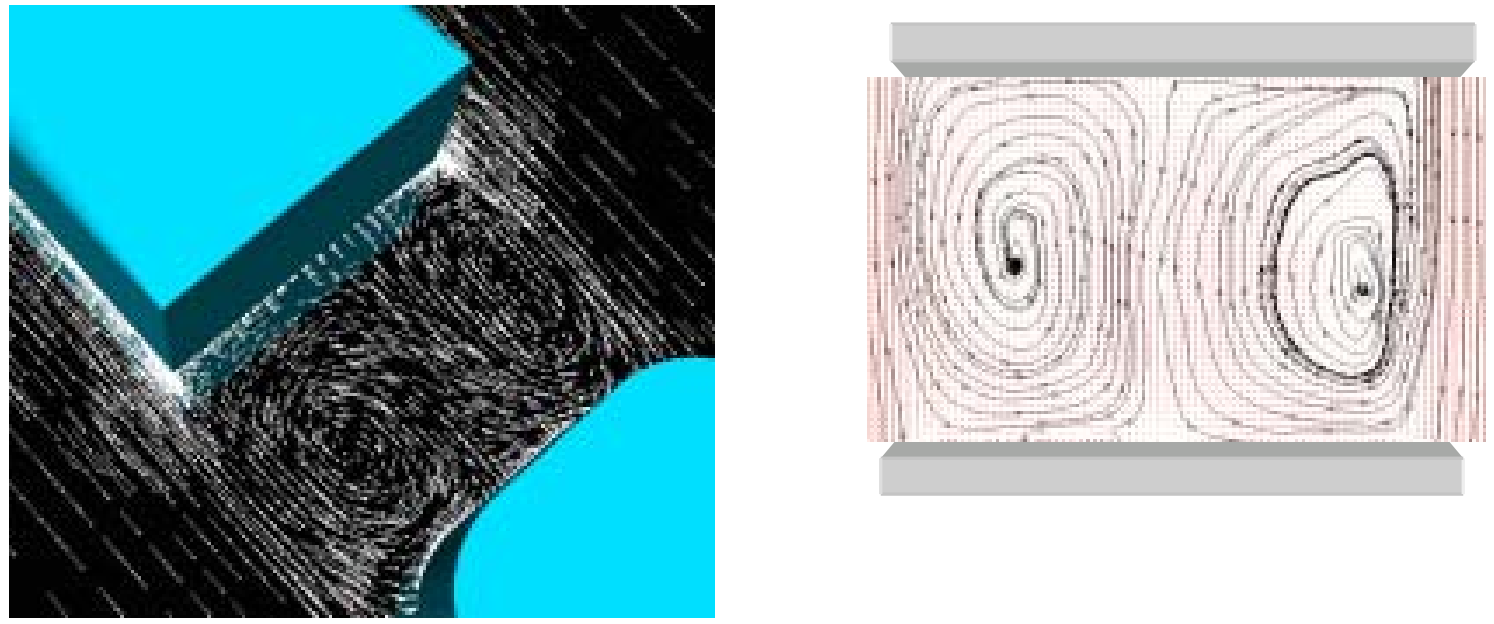

a) Computation (left image), USC experiment (right image), Symmetric condition
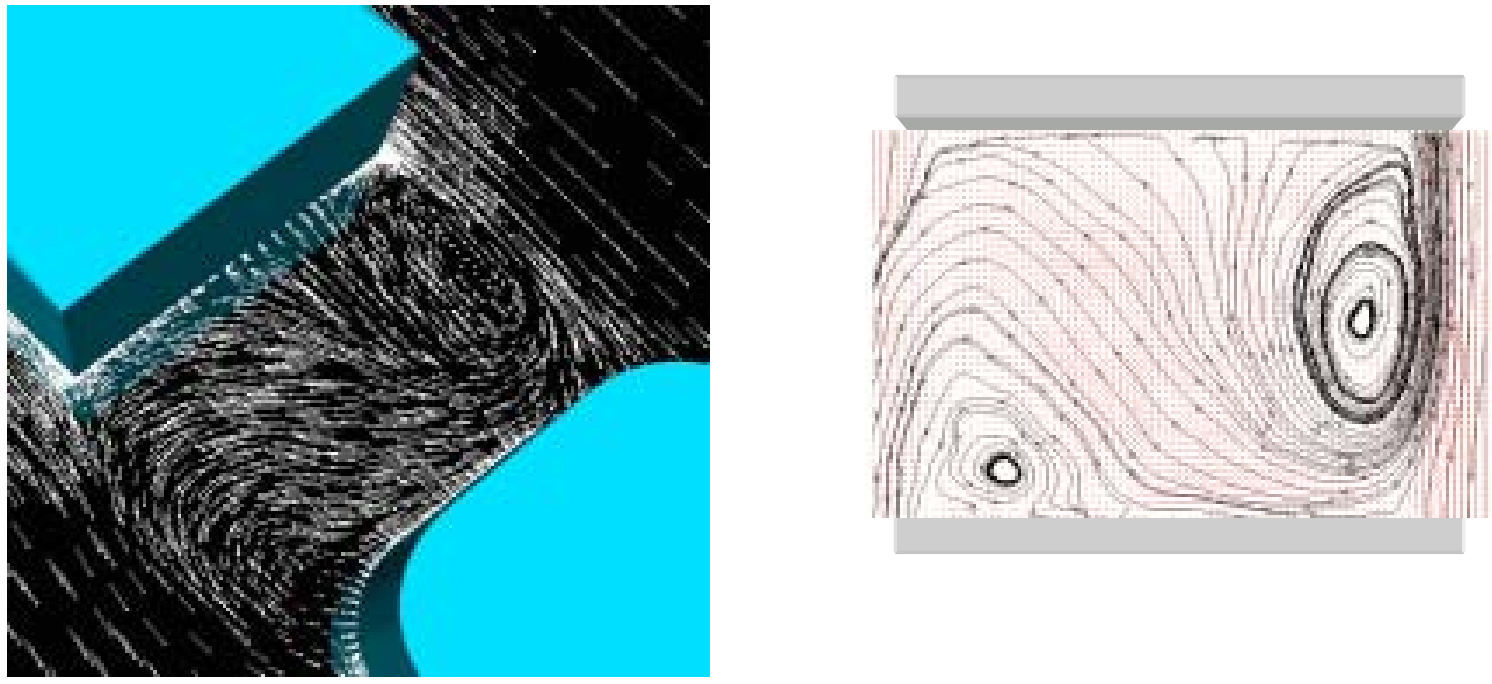

b) Computation (left image), USC experiment (right image), Asymmetric condition

Fig 10D - comparison of computed and USC experimental data at the gap mid-height, $0^{\circ}$ yaw, $k-w$ model, modified GTS geometry with 0.72 gap size

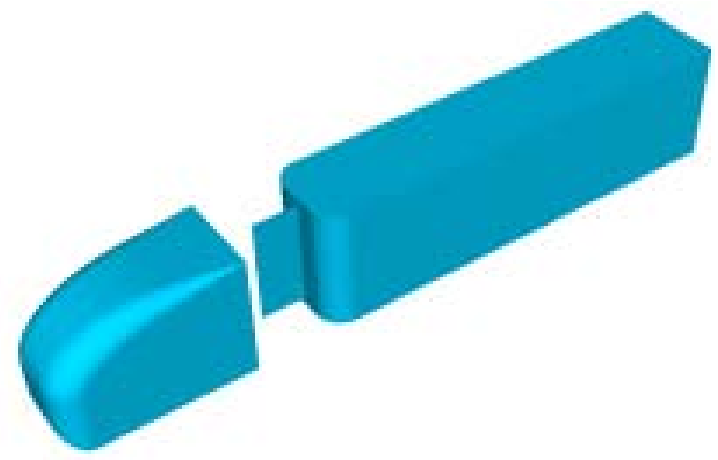

Fig 11D -add-on device (splitter plate) to stabilize the gap flow. 


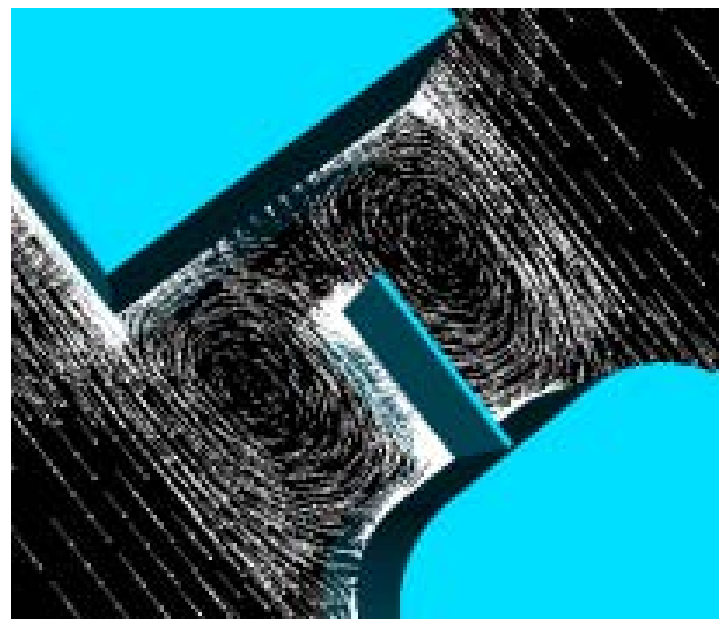

Fig 12D - Velocity vector field in the gap in the presence of the splitter plate, mid-height, $0^{\circ}$ yaw, k-w model, modified GTS geometry with 0.72 gap size

\section{Discovery Experiment}

The goal of our discovery experiment in the small-scale NASA Ames 3'x4' wind tunnel is to provide an inexpensive and flexible means of testing and exploring various LLNL drag reduction concepts for heavy vehicles. The construction of the $1 / 16$ scaled wind tunnel model which is similar to the USC modified GTS and the proposed LLNL add-on devices have been completed. The initial flow visualization about the modified GTS indicates flow separation on the side and the top of the tractor (Figure 13D). It is crucial to minimize the flow separation since it could adversely impact the evaluation of add-on devices. The flow separations are minimized by tripping the flow. The overall flow structure around the modified GTS model is complex with 3-D flow patterns on the downstream side of the tractor under yaw conditions. The trailer top and downstream side have recirculation zones that cover a significant portion of the trailer. Currently, force and moment measurements are being obtained for the baseline configuration with and without add-on drag reducing devices.

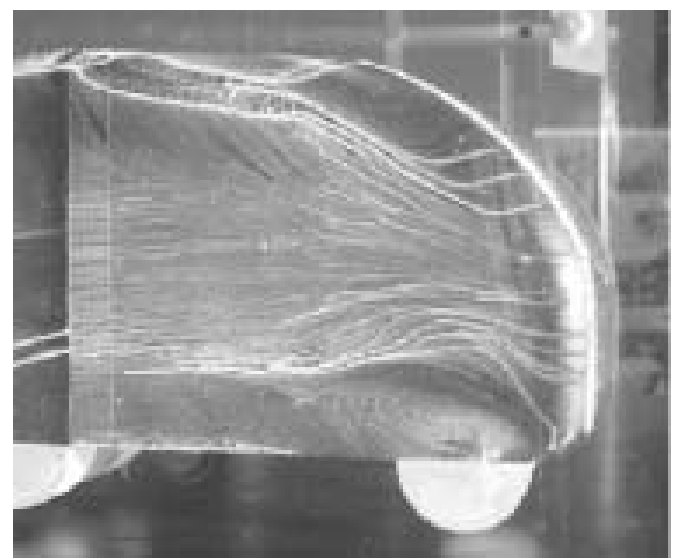

Fig 13D - 1/16 scaled wind tunnel model similar to USC modified GTS geometry

\section{Conclusions}

The steady and unsteady turbulent flow around the GTS and USC modified GTS was successfully completed. The RANS turbulence models at $0^{\circ}$ yaw, excluding the wake, performed reasonably well in predicting the surface properties and the overall flow behavior around the vehicle. The steady RANS turbulence models had difficulty capturing the right flow structure in the wake of the trailer. More sophisticated turbulence models, such as the hybrid RANS/LES model, should be investigated to improve the accuracy of the wake flow structure. Such a model is currently under development by LLNL.

The unsteady turbulent gap flow was successfully modeled compared to the USC experimental data. The symmetric and asymmetric flow states in the gap were properly represented. A new addon device was suggested and tested computationally to stabilize the gap flow and reduce the aerodynamic drag. 
The discovery experiment is under way at NASA Ames facility to provide better understanding of the flow structure associated with drag reducing add-on devices. Also, several add-on devices will be recommend as the outcome of this experiment to the DOE heavy vehicle project for full-scale road tests.

\section{$\underline{\text { References }}$}

[1] - Storms, B., et. al., "An Experimental Study of the Ground Transportation System (GTS) Model in the NASA Ames 7- by 10-Ft Wind Tunnel," NASA/TM-2001-209621, Feb. 2001.
[2] - Salari, K., McWherter-Payne, M., "Computational Flow Modeling of a Simplified Integrated Tractor-Trailer Geometry," SAND2003-3383.

[3] - Ortega, J.M., Dunn. T., McCallen, R., Salari, K., "Computational Simulation of a Heavy Vehicle Trailer Wake," United Engineering Foundation, Aerodynamics of Heavy Vehicles: Trucks, Buses, and Trains Conference, Monterey, CA, December 2-6, 2002. 


\title{
APPENDIX E
}

\section{Reynolds-Averaged Navier-Stokes Simulations of a Simplified Tractor/Trailer Configuration}

\author{
Principal Investigators: L. J. DeChant
}

Co-Investigators: C. J. Roy, J. L. Payne, B. Hassan

Sandia National Laboratories

P.O. Box 5800, MS 0825, Albuquerque, NM 87185-0825

(505)844-4250 ljdecha@sandia.gov

Technology Development Manager: Sidney Diamond

202-586-8032,sid.diamond@ee.doe.gov

Technical Program Manager: Jules Routbort

630-252-5065, routbort@anl.gov

Contractor: Sandia National Laboratories

Contract No.: DE-AC04-94AL85000

\section{Objective}

- Evaluate the use of Reynolds Averaged Navier-Stokes (RANS) to predict the aerodynamic drag on tractor/trailers. This goal will be accomplished by computing accurate numerical solutions to representative tractor/trailer geometries, then validating those computations by comparisons to experimental data. These validated models will then be used to assess drag reduction techniques which have the potential to reduce the net oil consumption and operating costs for the trucking industry.

\section{Approach}

- Compute solutions with varying mesh resolution for the Ground Transportation System (GTS) geometry.

- Assess the numerical accuracy of the computed solutions.

- Validate the models by comparison to experimental data for surface pressure, flowfield velocity, and drag coefficient obtained in the NASA-Ames $7 \times 10 \mathrm{ft}$ wind tunnel.

\section{Accomplishments}

- Completed a new GTS fine mesh with appropriate y+ values to allow the removal of every other point in each direction to provide a consistently coarsened mesh.

- Successfully completed both coarse grid (2.5 million cells) and fine grid (20 million cells) solutions on the GTS geometry using both the Spalart-Allmaras and Menter k-omega turbulence models. 
- Completed assessment of the numerical errors in the fine grid GTS simulations.

- Compared simulation results to experimental data for surface pressure, wake velocities, and drag coefficient.

- Generated new flowfield mesh on a truncated GTS geometry to allow more detailed resolution of the truck wake region and the assessment of unsteady turbulence modeling approaches.

- Developed a reduced-order model for bluff-body drag on simplified geometries.

\section{Future Directions}

- Compare steady RANS simulations on the truncated geometry to results for full geometry.

- Compute additional steady RANS simulations on the truncated mesh with additional mesh resolution in the wake.

- Compute unsteady turbulent simulations of the GTS geometry using the truncated geometry.

- Continue to develop a reduced order model for bluff-body drag.

\section{Introduction}

In a typical class 8 tractor/trailer, energy losses due to rolling resistance and accessories increase linearly with vehicle speed, while energy losses due to aerodynamic drag increase with the cube of the speed. At a typical highway speed of $70 \mathrm{mph}$, aerodynamic drag accounts for approximately $65 \%$ of the energy output of the engine (McCallen et al 1999). Due to the large number of tractor/trailers on the US highways, even modest reductions in aerodynamic drag can significantly reduce domestic fuel consumption. Lower fuel consumption will result in a reduction in pollution emissions, and, more importantly, a reduced dependence on foreign oil.

The most common turbulence modeling approach for engineering applications involves solving the Reynolds-Averaged Navier-Stokes (RANS) equations. With this approach, the effects of the inherently three-dimensional and timevarying turbulent eddies on the mean flow are modeled and not simulated. The goal of this study is to assess the ability of steady-state RANS turbulence models to accurately predict the flowfield and aerodynamic drag for tractor/trailer configurations.

\section{Problem Formulation}

The configuration to be examined is the Ground Transportation System (GTS) studied experimentally at the NASA Ames research center (Storms et al 2001). The GTS geometry is a simplified tractor/trailer configuration which is mounted on four posts in the wind tunnel. A photograph of the GTS in the NASA Ames $7 \times 10 \mathrm{ft}$ wind tunnel is shown in Figure 1E. The GTS model is an approximately $1 / 8$ scale class 8 tractor/trailer configuration. The Reynolds number based on the trailer width $(\mathrm{W}=0.3238 \mathrm{~m})$ is 2 million, approximately one-half of full scale.

The GTS geometry, including the wind tunnel walls, is discretized using two mesh levels: a coarse mesh using 2.5 million grid points and a fine mesh using 20 million grid points. The grids are generated such that the wall $\mathrm{y}^{+}$values on the truck surface, supports, and lower wind tunnel wall are everywhere less than unity on the fine mesh. The side 
and top wind tunnel walls employ slip flow conditions. Structured meshes are employed using point-to-point match up at the block boundaries. The coarse mesh is domain-decomposed into 125 zones and is shown in Figure 2E. The fine mesh is decomposed into 1149 zones. Both the coarse and fine meshes are run on the massively parallel ASCI Red machine using one processor for each zone. The axes employed in the current effort are shown Figure 2E, with the $\mathrm{x}$ axis starting at the front of the tractor and running downstream, the $y$ axis in the vertical direction, and the $\mathrm{z}$ axis starting at the GTS symmetry plane and running spanwise towards the side wall.

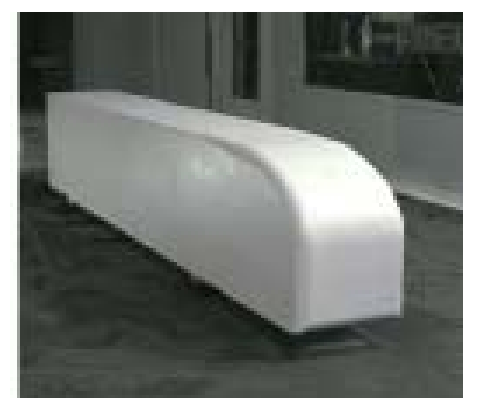

Figure 1E. GTS model in NASA Ames 7x10 ft wind tunnel

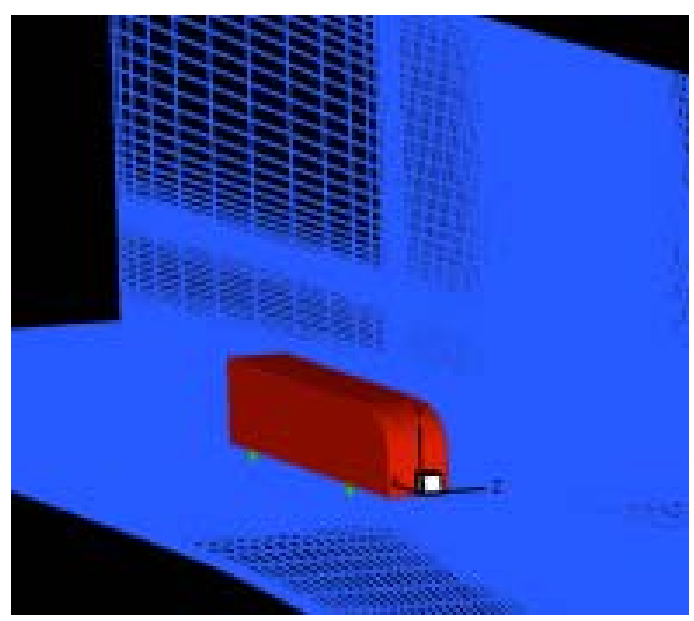

Figure 2E. Computational mesh on GTS geometry

In order to ensure that the simulated flow matches closely with the flow in the wind tunnel, a number of freestream conditions are matched. First, the inflow plane is set with the appropriate stagnation conditions of the tunnel shown in Table 1E. The back pressure at the simulated outflow plane is then adjusted until the reference pressure located at $(\mathrm{x} / \mathrm{W}=4.47, \mathrm{y} / \mathrm{W}=2.59$, and $\mathrm{z} / \mathrm{W}=-4.7)$ on the tunnel side wall reaches the wall reference pressure given in the table. The boundary layer on the bottom wall was measured in a tunnelempty configuration. Sample boundary layer profiles upstream of the GTS model from the simulation using the Menter k- $\omega$ model gave good agreement with the tunnel empty-profile from the experiment.

Table 1E. Freestream conditions used in the simulations

\begin{tabular}{|l|l|}
\hline Tunnel Condition & Value \\
\hline Stagnation Pressure & $102,653.2 \mathrm{~N} / \mathrm{m}^{2}$ \\
\hline $\begin{array}{l}\text { Stagnation } \\
\text { Temperature }\end{array}$ & $282.06 \mathrm{~K}$ \\
\hline $\begin{array}{l}\text { Wall Reference } \\
\text { Pressure }\end{array}$ & $97,582 . \mathrm{N} / \mathrm{m}^{2}$ \\
\hline $\begin{array}{l}\text { Reference Mach } \\
\text { number }\end{array}$ & 0.27 \\
\hline Back Pressure & $\begin{array}{l}100,136.0 \\
\mathrm{~N} / \mathrm{m}^{2}\end{array}$ \\
\hline $\begin{array}{l}\text { Tunnel Floor BL } \\
\text { Thickness }\end{array}$ & $0.053 \mathrm{~m}$ \\
\hline Wall Temperature BC & adiabatic \\
\hline
\end{tabular}

\section{$\underline{\text { Numerical Formulation }}$}

The CFD code is SACCARA, the Sandia Advanced Code for Compressible Aerothermodynamics Research and Analysis, and was developed from a parallel distributed memory version (Wong et al. 1995) of the INCA code, originally written by Amtec Engineering. The SACCARA code employs a massively parallel distributed memory architecture 
based on multi-block structured grids. A number of code verification studies have been performed which give confidence that the code is free from coding mistakes including comparison to established numerical benchmark solutions and code to code comparisons (Roy et al 2000). The fine grid and coarse grid simulations presented herein were run in parallel on the ASCI Red parallel processing machine using 1149 and 125 processors, respectively.

Two turbulence models are examined in the current work: the one-equation eddy viscosity transport model of SpalartAllmaras (Spalart and Allmaras 1994) and the Menter k- $\omega$ model (Menter 1994). The strengths and limitations of these models are described in the indicated references. The Menter k- $\omega$ model is a hybrid model which uses a blending function to combine the best aspects of both the $k-\omega$ and the $k-\varepsilon$ turbulence models. In both cases, the turbulence models were integrated to the wall in order to avoid model validation issues associated with wall functions.

\section{Numerical Accuracy}

The solutions are marched (iterated) in pseudo-time until a steady-state answer is obtained. Iterative convergence is assessed by examining the steady-state residuals of the momentum equations. The steady-state residual is defined by plugging the solution at the current iteration into the discretized form of the steady-state governing equations (omitting the time derivative). The iterative error in the solution tends to drop in a similar fashion as the residual. The solutions were converged by approximately seven orders of magnitude.
The discretization error is estimated by generating solutions on two mesh levels. Since the coarse mesh is determined by eliminating every other gridline from the fine mesh, the grid is consistently refined throughout the entire domain, and Richardson extrapolation can be used to estimate the exact solution. This extrapolated solution is then used to judge the error in the fine grid solutions. While the coarse and fine grid results for surface pressure on the front of the tractor do show some sensitivity to grid refinement, the estimated error in the fine grid solution is approximately \pm 0.05 $\Delta \mathrm{Cp}$. Coarse and fine grid results for the base of the trailer are shown in Figure $3 \mathrm{E}$, with the maximum estimated error to be less than $\pm 0.01 \Delta \mathrm{Cp}$ (note the expanded scale for $\Delta \mathrm{Cp}$ ). The SpalartAllmaras model was only run on the fine mesh; however, the spatial discretization error is expected to be similar to that of the Menter model.

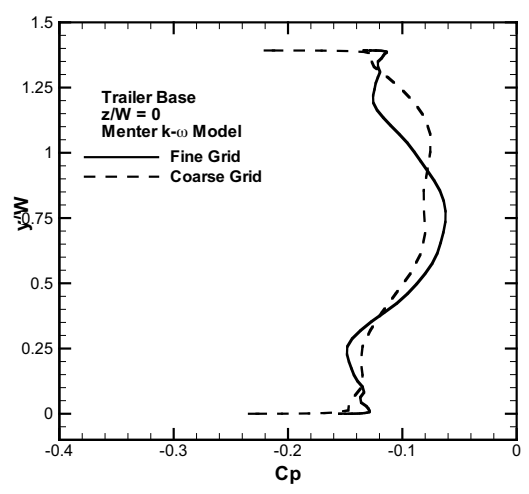

Figure 3E. Pressure on base of trailer (coarse and fine meshes)

\section{$\underline{\text { Surface Comparisons }}$}

The surface pressure for the two turbulence models using the fine mesh is compared to experimental data (Storms et al 2001) on the front of the tractor in Figure 4E. Both simulations show good agreement with the experimental data. 
Simulation results are presented for the base of the trailer in Figure 5E. In this case, the Menter k- $\omega$ model does a reasonable job of matching the pressure levels, while the Spalart-Allmaras model significantly underpredicts the pressure on the base.

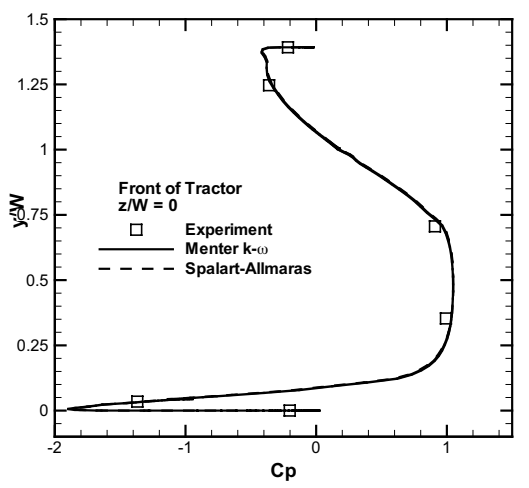

Figure 4E. Surface pressures on front of tractor

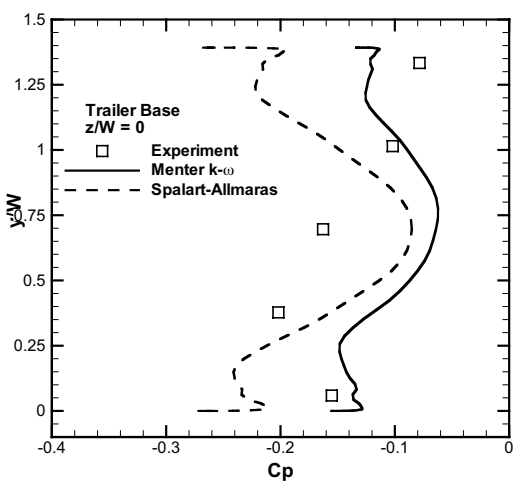

Figure 5E. Surface pressure on trailer base

\section{Aerodynamic Drag}

Aerodynamic drag predictions using the two turbulence models as well as the experimentally measured drag are presented in Table 2E. These drag results (both predicted and experimental) are for the GTS model only and do not include the support posts. The Menter k- $\omega$ results are approximately $7.5 \%$ higher than the experimental value, while the Spalart-Allmaras results are nearly 50\% high. The overprediction of the drag with the Spalart-Allmaras model is due to the poor prediction of the base pressure. Also shown in the table is the estimated uncertainty in the experimental drag coefficient (Storms et al 2001), and the estimated numerical error for the fine grid Menter $k-\omega$ simulation. This numerical error estimate is determined by performing Richardson extrapolation using the coarse and fine grid drag coefficients, 0.474 and 0.298 , respectively. The resulting extrapolated value for the drag coefficient using the Menter k- $\omega$ model is 0.239 , which is essentially within the experimental uncertainty bounds.

Table 2E. Drag coefficients

\begin{tabular}{|l|l|l|}
\hline & $\begin{array}{l}\text { Drag } \\
\text { Coeff., } \\
\mathrm{C}_{\mathrm{D}}\end{array}$ & $\begin{array}{l}\text { Estimated } \\
\text { Uncertainty/Error }\end{array}$ \\
\hline $\begin{array}{l}\text { Experiment } \\
\text { (Storms et al } \\
\text { 2001) }\end{array}$ & 0.25 & \pm 0.01 \\
\hline Menter k- $\omega$ & 0.298 & \pm 0.06 \\
\hline Spalart-Allmaras & 0.413 & -- \\
\hline
\end{tabular}

\section{Field Comparisons}

Velocity data are available from PIV measurements performed at the NASA Ames $7 \times 10 \mathrm{ft}$ wind tunnel (Storms et al 2001). These PIV data represent a timeaveraged picture of the flow in the wake regions immediately behind the trailer base. Figure 6E gives streamlines based on the PIV data in a vertical streamwise cut through the wake $(\mathrm{z} / \mathrm{W}=0)$. The flow is from left to right, with the base of the trailer shown on the left; the PIV window is also shown in the figure. A large, counter-clockwise-rotating vortex is centered at approximately $\mathrm{x} / \mathrm{W}=8$, $\mathrm{y} / \mathrm{W}=0.4$. Also, the presence of a clockwise-rotating vortex is suggested by the vertical nature of the streamlines in the upper right-hand corner of the PIV 
window. A similar view of the streamlines from the fine grid computations using the Menter k- $\omega$ model is shown in Figure 7E. These Menter k- $\omega$ computations predict a more symmetric pair of vortices than is indicated from the experimental data. The location of the experimental PIV window is shown in the figure for reference. Streamlines for the SpalartAllmaras model are presented in Figure 11E, showing a much shorter recirculation zone than the Menter model. This shorter recirculation zone produces larger velocities in the outer flow as the flow accelerates around the wake. The higher velocities result in lower pressures and hence higher drag (see Table 2E). Although not depicted, comparison to experimental PIV results for a horizontal streamwise cut through the wake $(\mathrm{y} / \mathrm{W}=0.696)$ showed that the location of the vortices was accurately predicted by the RANS model

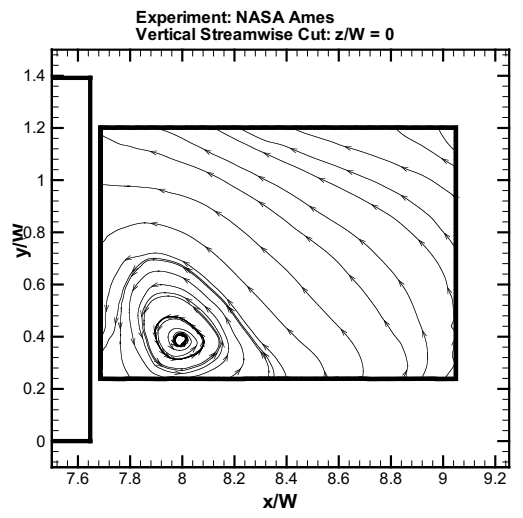

Figure 6E. Experimental streamlines: vertical streamwise cut $(\mathrm{z} / \mathbf{W}=\mathbf{0})$

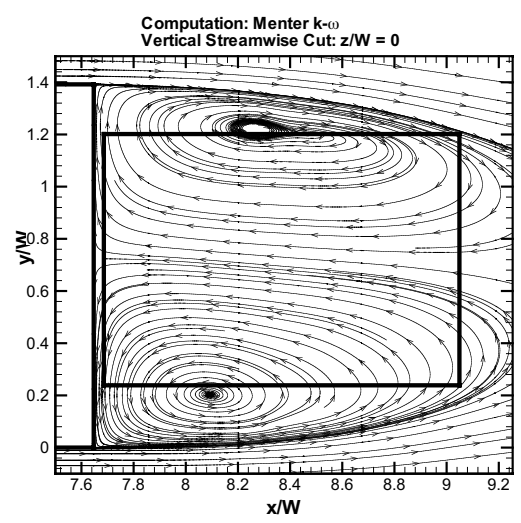

Figure 7E. Computational streamlines: vertical streamwise cut $(\mathrm{z} / \mathrm{W}=\mathbf{0})$

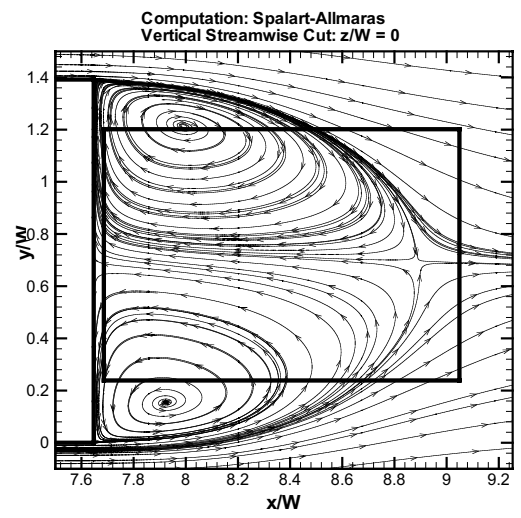

Figure 8E. Computational streamlines: vertical streamwise cut $(z / \mathbf{W}=0)$

\section{Truncated Geometry}

A truncated GTS mesh was generated which omits the upstream wind tunnel and the front "tractor" section of the GTS. Steady-state RANS simulations on this geometry are underway and will be completed early in Fiscal Year 2004. Our goal here is to develop the minimum computational problem that adequately represents the large-scale separation effects in the flow and, thereby, recovers the dominant drag component. Simulations are to be performed on both supercomputer and multiple workstation class platforms. The ability to model these flows on widely avaliable work station clusters (10-20 processors) is of 
particular interest, since it can be expected that many end users will have this level of computing resources currently available.

\section{Reduced Order Modeling}

Here, the self-similar, far-field, turbulent wake models are extended to estimate the 2-d, axi-symmetric and (simple) 3-d drag coefficient for a range of bluff body problems. The self-similar wake velocity defect that is normally independent of the initial condition, e.g. bluff body geometry, is modified using a GramCharlier series approach to retain the initial condition information. Preliminary results indicate that drag estimates computed using this method are within approximately $20 \%$ as compared to published values for flows with large separation. The potential value of this method is as a way to utilize poorly resolved simulation results to provide an inexpensive estimate of body drag or as the basis of a physically consistent correlation scheme. This methodology may be of use as a supplement to CFD and experimental solutions in reducing the heavy computational and experimental burden of estimating drag coefficients for blunt body flows for preliminary design type studies.

\section{$\underline{\text { Conclusions }}$}

Steady-state RANS simulations were conducted for the flow over the GTS geometry. The numerical accuracy of the computed flowfields was assessed by performing the computations on multiple grids. Simulation results using the Menter k- $\omega$ turbulence model gave good agreement with the experimental data for surface pressure, field velocities in the wake, and drag coefficient; however, this model predicted a more symmetric pair of counter-rotating vortices in the vertical streamwise plane than was indicated in the experiment. These results suggest that the Menter $\mathrm{k}-\omega$ model can accurately predict the drag for tractor/trailer configurations when performed on highly refined meshes using integration to the wall, although some time-averaged flow structures are not captured. Simulation results with the Spalart-Allmaras turbulence model showed good agreement with experimental data for the surface pressure in the attached flow regions, but significantly underpredicted the pressure in the base region. As a result, the Spalart-Allmaras model overpredicted the drag coefficient by nearly $50 \%$. Initial development of reduced-order drag prediction models were also introduced, with the preliminary results yielding drag estimates within $20 \%$ of established values.

\section{References}

McCallen R, Couch R, Hsu J, Browand F, Hammache M, Leonard A, Brady M, Salari K, Rutledge W, Ross J, Storms B, Heineck JT, Driver D, Bell J, Zilliac G (1999) Progress in reducing aerodynamic drag for higher efficiency of heavy duty trucks (class 7-8). SAE Paper 1999-01-2238.

Menter FR (1994) Two-equation eddy-viscosity turbulence models for engineering applications. AIAA Journal 32: 1598-1605

Roe PL (1981) Approximate Riemann solvers, parameter vectors, and difference schemes. Journal of Computational Physics 43: 357-372

Roy CJ, McWherter-Payne MA, Oberkampf WL (2000) Verification and validation for laminar hypersonic flowfields. AIAA Paper 2000-2550

Spalart PR, Allmaras SR. (1994) A one-equation turbulence model for aerodynamic flows. La Recherche Aerospatiale 1: 5-21 
Storms BL, Ross JC, Heineck JT, Walker SM, Driver DM, Zilliac GG (2001) An experimental study of the ground transportation system (GTS) model in the NASA Ames 7- by 10-ft wind tunnel. NASA TM2001-209621.

Wong CC, Soetrisno M, Blottner FG, Imlay ST, Payne JL (1995) PINCA: a scalable parallel program for compressible gas dynamics with nonequilibrium chemistry. Sandia National Laboratories Report SAND 94-2436, Albuquerque, NM
Yee HC (1987) Implicit and symmetric shock capturing schemes. NASA-TM-89464 


\title{
APPENDIX F
}

\section{Commercial CFD Code Validation for External Aerodynamics Simulations of Realistic Heavy-Vehicle Configurations}

\author{
Principal Investigator: $W$. D. Pointer \\ Argonne National Laboratory \\ 9700 S. Cass Avenue, NE-208, Argonne, IL 60439 \\ (630) 252-1052; fax: (630) 252-4500; e-mail: dpointer@anl.gov \\ Technology Development Manager: Sid Diamond \\ 202-586-8032,sid.diamond@ee.doe.gov \\ Technical Program Manager: Jules Routbort \\ 630-252-5065,rroutbort@anl.gov
}

Contractor: Argonne National Laboratory

Contract No.: W-31-109-ENG-38

\section{Objective}

- Evaluate capabilities in standard commercial computational fluid dynamics (CFD) software for the prediction of aerodynamic characteristics of a conventional U.S. Class 8 tractor-trailer truck.

- Develop "best practice" guidelines for the application of commercial CFD software in the design process of Class 8 vehicles.

\section{Approach}

- Develop computational models of the experiments completed in the NASA Ames Laboratory's $7 \mathrm{ft}$. by $10 \mathrm{ft}$. wind tunnel using the Generic Conventional Model (GCM).

- Compare the predictions of the computational models with experimental measurements of vehicle aerodynamic drag force and pressure field distributions.

\section{Accomplishments}

- Experimental measurements and computational predictions of the vehicle drag coefficient agree within less than 1 percent in the best case simulations.

- Experimental measurements and computational predictions of the pressure distribution along the surface of the vehicle agree within the experimental error.

\section{Future Direction}

- Continue development of "best practice" guidelines through comparison of computational predictions with experimental measurement for the GCM as well as grid, model geometry, and turbulence model studies. 
- Evaluate capabilities for prediction of aerodynamic drag in cases in which there is a cross-wind component (i.e., consider wind tunnel experiments where the vehicle is placed at some yaw angle).

- Consider alternate GCM configurations using various add-on devices to examine capabilities for the prediction of changes in drag coefficient.

\section{Introduction}

The issue of energy economy in transportation has grown beyond traditional concerns over environment, safety and health to include new concerns over domestic energy security. In collaboration with the U. S. Department of Energy Office of FreedomCAR and Vehicle Technologies' Working Group on Aerodynamic Drag of Heavy Vehicles, Argonne National Laboratory is developing guidelines for the accurate prediction of heavy vehicle aerodynamic drag coefficients using commercial Computational Fluid Dynamic (CFD) software. In these studies, computational predictions from the commercial CFD code Star-CD [1] will be compared with detailed velocity, pressure and force balance data from experiments completed in the $7 \mathrm{ft}$. by $10 \mathrm{ft}$. wind tunnel at NASA Ames [3,4] using a Generic Conventional Model (GCM) that is representative of typical current-generation class 8 tractor-trailer geometries. Initial evaluations have focused on the effects of selection of computational mesh size parameters and turbulence modeling strategies on the accuracy of the drag coefficient prediction.

\section{Generic Conventional Model}

The Generic Conventional Model (GCM) is a simplified representation of a conventional U.S. tractor-trailer truck. The model is $1 / 8$ th scale and can be configured in four different geometries as illustrated in Fig. 1F. The nominal, or Standard Truck, configuration is a representative model of a currentgeneration U. S. conventional class 8 tractor-trailer truck. Alternate GCM configurations include the addition of a loboy device under the length of the trailer, a full fairing between the cab and the trailer,

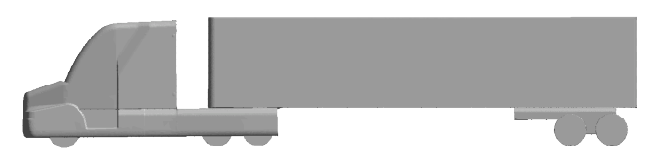

(a)

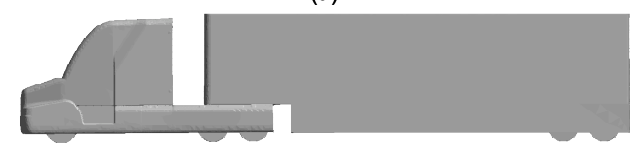

(b)

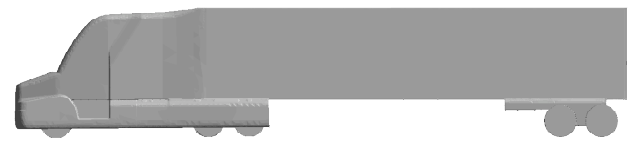

(c)

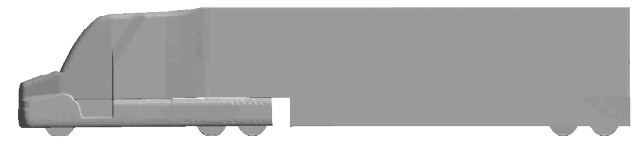

(d)

Fig. 1F. Four Configurations of the Generic Conventional Model: (a, Standard Truck, (b) Standard Truck with Lo-Boy Trailer, (c) Faired Truck, (d, Faired Truck with Lo-Boy Trailer.

and the combination of the fairing and lowboy device.

\section{Computational Model}

The computational model employed in these studies was developed using the ES-Aero tool for aerodynamic drag simulation that is available as part of the Star-CD software package. The mesh is developed using a semi-automated process that progresses in eight stages:

1. A three-dimensional hexahedral mesh is created that completely fills the volume of the wind tunnel. 


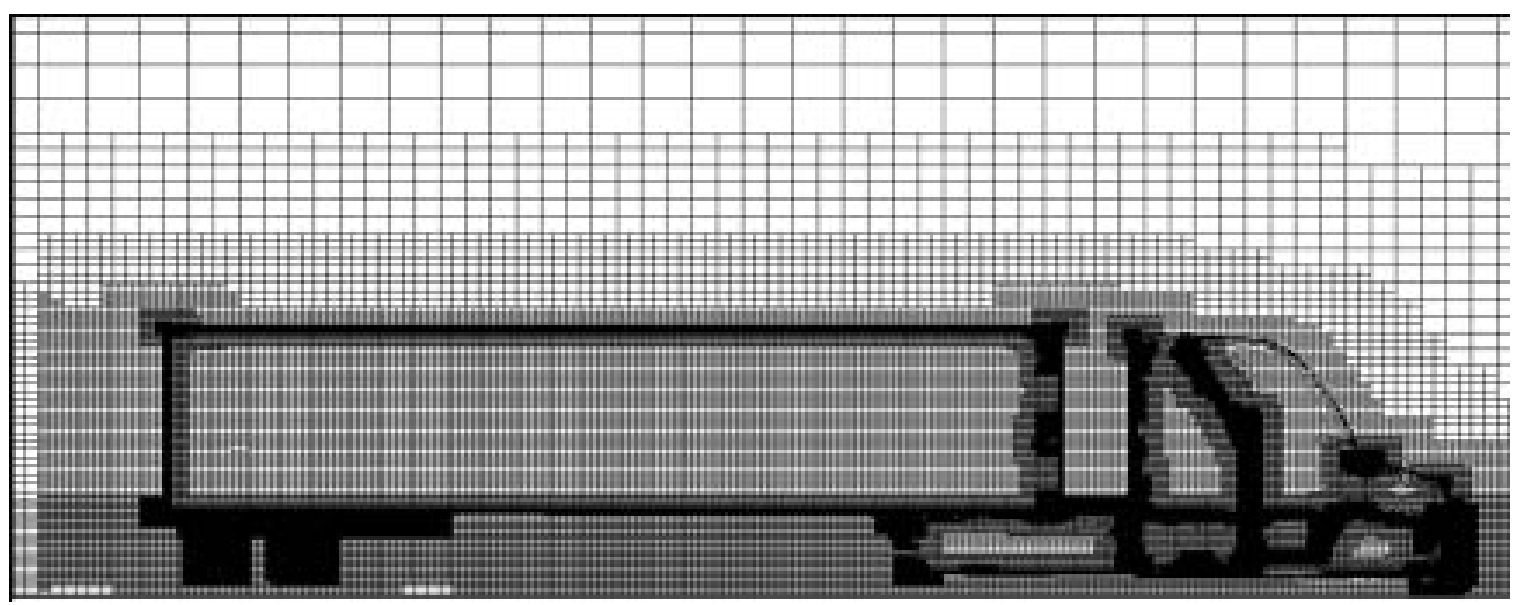

Fig. 2F. Two-dimensional projection of the near-vehicle region of the computational mesh employed in these studies.

2. The mesh is refined in successively smaller zones surrounding the vehicle until the mesh adjacent to the vehicle surface reaches the prescribed near-vehicle cell size. The resulting unstructured mesh of hexahedral cells exhibits 2-to-1 matching at all unstructured interfaces.

3. The near-vehicle mesh is locally refined based upon features of the vehicle surface definition. Local refinement limits are determined through the near-wall cell size parameter and direct user identification.

4. The surfaces that define the vehicle are "wrapped" by projecting the refined hexahedral mesh onto the surface. The components of the vehicle are merged into a single surface, and a quadrilateral surface definition is created.

5. The quadrilateral surface definition is volumetrically expanded to create a sub-surface.

6. The sub-surface is used to cut away the parts of the hexahedral mesh that fall within a short distance of the vehicle surface.
7. A brick and prism extrusion layer is created to fill the gap between the sub-surface and the quadrilateral surface definition. Thus, the polyhedral trimmed cells that are generated when the vehicle shape is cut from the mesh are not in the critical boundary layer region of the problem.

8. Upon completion of the basic mesh, the wake and ground layer regions are automatically further refined to better capture important flow features.

In this study, the generated mesh has a nearwall cell size of $8.0 \mathrm{~mm}$. The minimum cell size used in local refinements is $0.5 \mathrm{~mm}$, and a minimum of 16 points are required to define any full circle. In addition to automatic refinements, cells adjacent to the surface are refined to a size of $2.0 \mathrm{~mm}$ in order to preserve the quality of the surface in the wrapping stage and improve the quality of the trimmed cells. The extrusion layer consists of two layers of brick and prism cells where the outer layer has a thickness of $1.0 \mathrm{~mm}$ and the inner layer has a thickness of $0.5 \mathrm{~mm}$. A sample computational mesh is shown in Fig. 2F.

In all simulations discussed herein, the GCM is centered at zero yaw on the floor of 
a wind tunnel test section that is $2.133 \mathrm{~m}$ (7 ft.) tall by $3.048 \mathrm{~m}$ (10 ft.) wide. Since the GCM is approximately $2.5 \mathrm{~m}$ long, a total test section length of $10.0 \mathrm{~m}$ is assumed, where one model length is included upstream of the model and two model lengths are included downstream of the model. Based upon a Reynolds number of 1.1 million, a uniform velocity of $51.45 \mathrm{~m} / \mathrm{s}$ is enforced at the inlet boundary. A uniform pressure condition is applied at the outlet boundary. In these studies, the surface of the standard configuration GCM is defined using approximately 500,000 triangular surface elements that are based upon CAD representations taken from optical scans of the actual model.

\section{Mesh Sensitivity Studies}

The Star-CD external aerodynamics analysis tool ES-Aero uses a local feature-size-based automatic meshing system to build a partially-unstructured, primarily-hexahedral computational mesh. In the generation process, a bulk near-vehicle cell size and a minimum cell size for local feature-based refinement regions, or near-wall cell size, are specified. In these studies, the effects of changes of these two parameters on predicted drag coefficient are evaluated.

For each steady-state simulation, 3000 iterations were calculated using Star-CD's standard conjugate gradient solver and the PISO predictor-corrector algorithm. In these simulations, the standard high Reynolds number two-equation turbulence model and a logarithmic wall function are employed for prediction of turbulent kinetic energy and dissipation. Convergence criteria were set to insure that all cases would reach 3000 iterations before stopping. However, all residuals reach the normal stopping criteria of $10^{-3}$ well before the 3000th iteration and are no larger than $2.0 \times 10^{-4}$ at the 3000th iteration. In addition to standard velocity and mass parameter residual monitoring, the drag coefficient of the vehicle is monitored as the solution develops to insure that the drag coefficient reaches a converged solution. In order to reduce computational cost for these studies, only half of the GCM is included in the model and a symmetric boundary condition is employed at the centerline.

The effects of the near-vehicle cell size parameter on the accuracy of drag coefficient prediction were first considered. The near-vehicle cell size was varied in size from $6 \mathrm{~mm}$ to $16 \mathrm{~mm}$ in five cases. The results, shown in Table $1 \mathrm{~F}$, indicate that the drag coefficient can be predicted within 1.7 percent using a cell size of $6 \mathrm{~mm}$ and 10.5 percent using a cell size of $16 \mathrm{~mm}$. Further reductions in near-vehicle cell size are not possible without violating the memory limits of the 32-bit system used in the development of these computational meshes. Further increases in the near-vehicle cell size will result in cells that are two large to capture significant vehicle features such as the rear wheel axels.

The effect of the near-wall cell size parameter on the accuracy of drag coefficient prediction was also considered. In this case, the near-vehicle cell size is set to $8 \mathrm{~mm}$ and the minimum cell size for local refinement was reduced from $1 \mathrm{~mm}$ to $0.5 \mathrm{~mm}$. This increases the number of computational elements from $3,282,426$ to $4,264,232$ and the total CPU time used from 610,958 seconds to 703,027 seconds. The change in the near-wall refinement parameter results in a reduction in the error

Table 1F. Effects of Near-Vehicle Cell Size Parameter on Accuracy of Drag Coefficient Prediction.

\begin{tabular}{|c|c|c|}
\hline $\begin{array}{c}\text { Near-Vehicle } \\
\text { Cell Size } \\
(\mathbf{m m})\end{array}$ & $\begin{array}{c}\text { Predicted } \\
\text { Drag } \\
\text { Coefficient }\end{array}$ & $\begin{array}{c}\text { Error in Drag } \\
\text { Coefficient }\end{array}$ \\
\hline experiment & 0.398 & 12.0 \\
\hline 16 & 0.449 & 10.3 \\
\hline 12 & 0.441 & 4.9 \\
\hline 10 & 0.418 & 4.2 \\
\hline 8 & 0.415 & 1.7 \\
\hline 6 & 0.405 & \\
\hline
\end{tabular}


of the drag coefficient prediction from 4.2 percent to 1.0 percent.

\section{Turbulence Model Sensitivity}

In order to evaluate the sensitivity of the solution to the turbulence model employed, a study was completed using three different turbulence models that are included as standard options in the Star-CD software package: the standard high-Reynolds number k-epsilon model with logarithmic wall function, the Menter k-omega SST model [5], and the renormalization group (RNG) formulation of the k-epsilon model [6]. The standard k-epsilon model and the SST model are identical in the far field, but the SST model incorporates additional detail in the near-wall region. The SST model should be more sensitive to separation, but the two should show reasonable agreement. The RNG model is similar to the standard kepsilon model, but includes an additional term to account for the mean flow distortion of the dissipation. With all three models,

Table $2 F$. Results of the evaluation of twoequation turbulence models for prediction of tractor-trailer truck drag coefficients.

\begin{tabular}{|c|c|c|}
\hline $\begin{array}{c}\text { Turbulence } \\
\text { Model }\end{array}$ & $\begin{array}{c}\text { Predicted Drag } \\
\text { Coefficient }\end{array}$ & $\begin{array}{c}\text { Percent Error in } \\
\text { Prediction }\end{array}$ \\
\hline Experiment & 0.398 & -- \\
\hline $\begin{array}{c}\text { High-Reynolds } \\
\text { Number k-epsilon } \\
\text { Model }\end{array}$ & 0.402 & 1.0 \\
\hline $\begin{array}{c}\text { Menter k-_SST } \\
\text { model }\end{array}$ & 0.401 & 0.8 \\
\hline RNG model & 0.389 & 2.3 \\
\hline
\end{tabular}

logarithmic wall functions are employed for boundary layer modeling. All simulations use a highly- refined mesh with a nearvehicle cell size of $8.0 \mathrm{~mm}$ and a minimum cell size of $0.5 \mathrm{~mm}$. As in previous studies, only one symmetric half of the vehicle is considered to reduce the computational requirements. Again, 3000 iterations are completed for each case and the convergence of the drag coefficient is monitored. Results of the study, as summarized in Table $2 \mathrm{~F}$, indicate that the Menter k-omega SST model provides a slight performance improvement over the other models.

\section{Half Vehicle Versus Full Vehicle}

In order to evaluate the effects of considering only half of the vehicle rather than the full vehicle, two models were created using the full vehicle geometry. These models use the same mesh parameter settings as the two coarsest models considered in the mesh sensitivity study, with near-wall cell sizes of $12 \mathrm{~mm}$ and 16 $\mathrm{mm}$. As in all previous studies, 3000 iterations were completed for each steadystate simulation and the convergence of the drag coefficient was monitored. As shown in Table 3F, drag coefficient predictions show a slight improvement in agreement with experimental measurements when the full vehicle model is used.

\section{Pressure Field Comparisons}

Since the drag coefficient is an integrated quantity, comparison of the simulation predictions of surface pressure distribution with the experimentally measured surface pressure distribution provides another

Table 3F. Comparison of drag coefficient predictions from half-vehicle and full-vehicle models.

\begin{tabular}{|c|c|c|c|c|}
\hline & \multicolumn{2}{|c|}{ Half-Vehicle } & \multicolumn{2}{c|}{ Full-Vehicle } \\
\hline $\begin{array}{c}\text { Near-Vehicle } \\
\text { Cell Size } \\
(\mathbf{m m})\end{array}$ & $\begin{array}{c}\text { Predicted Drag } \\
\text { Coefficient }\end{array}$ & $\begin{array}{c}\text { Percent Error in } \\
\text { Prediction }\end{array}$ & $\begin{array}{c}\text { Predicted Drag } \\
\text { Coefficient }\end{array}$ & $\begin{array}{c}\text { Percent Error in } \\
\text { Prediction }\end{array}$ \\
\hline 16 & 0.449 & 12.0 & 0.441 & 10.3 \\
\hline 12 & 0.441 & 10.3 & 0.426 & 6.7 \\
\hline
\end{tabular}




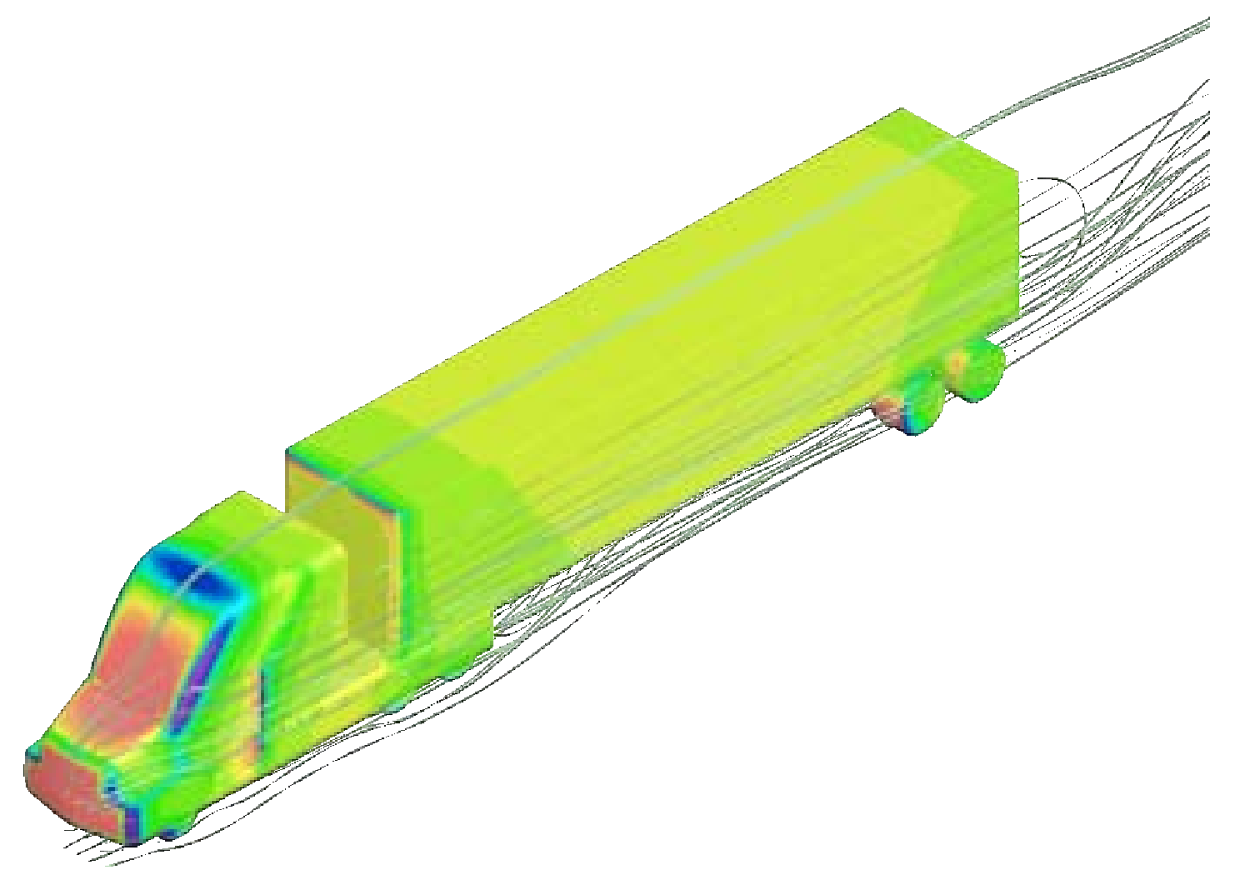

Fig 3F. Surface contour plot showing the predicted surface pressure distribution along the surface of the GCM. Areas shown in red are areas of peak positive pressure and areas shown in blue are areas of peak negative pressure. Selected flow streamlines are also shown to illustrate the movement of air over the vehicle.

measure of the accuracy of the simulation. The pressure distribution along the vehicle surface from the case using a half-vehicle model with a near-vehicle cell size of $8 \mathrm{~mm}$, a near-wall cell size limit of $0.5 \mathrm{~mm}$ and the Menter k-_ SST model is shown in Fig. 3F. The comparison of the predicted pressure distribution along the vehicle centerline with experimental measurements, as shown in Fig. 4F, indicates that the surface pressure distribution is predicted with reasonable accuracy. A more detailed comparison of the pressure distribution along the rear face of the trailer, which is typically, the most difficult region to predict accurately, is shown in Fig. 5F.

\section{Conclusions}

In these studies, the predictions of heavy vehicle aerodynamic characteristics generated using standard mesh generation and turbulence modeling options within the

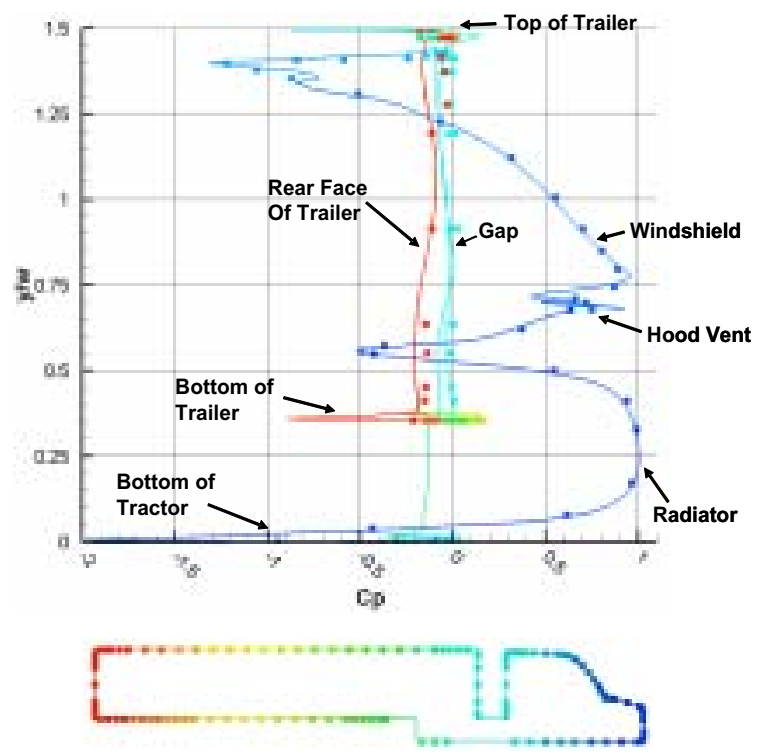

Fig. $\mathbf{4 F}$. Comparison of predicted (line) and measured (dot) pressure distributions along the centerline of the Generic Conventional Model. As shown in the outline of the GCM, shading indicates axial position with blue indicating the grill and bumper area and red indicating the rear face of the trailer. 
commercial computational fluid dynamics (CFD) code Star-CD have been compared with wind tunnel experiments using the Generic Conventional Model (GCM). The computational predictions show good agreement with the experimental measurements of the drag coefficient. The predicted surface pressure distribution also show good agreement with measured values with the maximum error occurring along the rear faces of the cab and the trailer. While the results of these studies are promising, they should not be viewed as conclusive, since the capabilities of the software for the prediction of changes in drag coefficient with changes in vehicle geometry are not yet known. In foreseeable applications within the truck manufacturing industry, the ability to predict changes in aerodynamic characteristics resulting from small changes in vehicle design is likely to be more valuable than the ability to predict the absolute value of the drag coefficient.

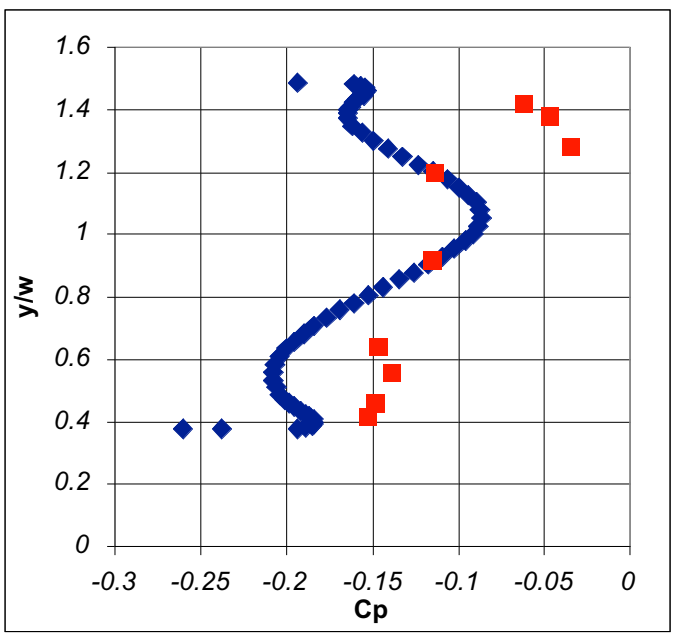

Fig. 5F. Comparison of predicted (diamond) and measured (square) pressure distributions along the centerline of the rear face of the trailer.

\section{Future Work}

The next phase of this project will duplicate the analyses discussed herein for each of the alternative configurations of the GCM. Additionally, the capability of the commercial CFD software to predict the aerodynamic characteristics of the Standard Truck configuration when a cross-wind component is present will be evaluated by comparison with measurements from experiments using the GCM placed at a set yaw angle within the $7 \mathrm{ft}$. by $10 \mathrm{ft}$. wind tunnel. These future studies will provide information on the capability of the code to predict changes in the aerodynamic characteristics of a heavy vehicle resulting from changes in design or environment.

\section{REFERENCES}

1. Star-CD, version 3.150A, CD-Adapco Group, Melville, NY.

2. PowerFLOW, version 3.4, Exa Corporation, Lexington, MA.

3. Dale Satran, "An Experimental Study of the Generic Conventional Model (GCM) in the NASA Ames 7-by-10-Foot Wind Tunnel," United Engineering Foundation Conference on The Aerodynamics of Heavy Vehicles: Trucks, Buses, and Trains, Monterey, CA, Dec 2-6, 2002.

4. J. T. Heineck, Stephen Walker, Dale Satran, "The Measurement of Wake and Gap Flows of a 1/8th Scale Generic Truck Using Three-Component Particle Image Velocimetry," United Engineering Foundation Conference on The Aerodynamics of Heavy Vehicles: Trucks, Buses, and Trains, Monterey, CA, Dec 2-6, 2002.

5. Menter, F.R.,'Zonal Two Equation k-_ Turbulence Models for Aerodynamic Flows"in 24th Fluid Dynamics Conference (Orlando), AIAA paper 932906, July 1993.

6. Yakhot, V., Orszag, S.A., Thangam, S., Gatski, T.B., and Speziale, C.G. 'Development of turbulence models for shear flows by a double expansion technique', Phys. Fluids, A4, No. 7, pp. 1510-1520, 1992. 


\section{APPENDIX G}

\section{Vortex Methods}

Principal Investigator: A. Leonard

Co-Investigator: M. Rubel, P. Chatelain

California Institute of Technology

1200 East California Blvd. MC 301-46, Pasadena, CA 91125

(626) 395-4465; tony@galcit.caltech.edu

Technology Development Manager: Sid Diamond

202-586-8032, sid.diamond@ee.doe.gov

Technical Program Manager: Jules Routbort

630-252-5065,routbort@anl.gov

Contractor: California Institute of California

Contract No.: DE-AC03-98EE50506

\section{Objective}

- Develop and demonstrate gridless flow field method for computationally modeling heavy vehicle aerodynamics.

\section{Approach}

- Improve and extend vortex method approach and demonstrate capabilities on generic shapes and Ground Transportation System geometry.

\section{Accomplishments}

- Developed treatment of spinning boundaries.

- Study of boundary elements has led to a method for representing near-wall vorticity.

- Developed new method for redistribution of vortex particles.

- Development of a "Closest Point Transform" algorithm for improved runtime efficiency.

- Continued improvements in adaptive time integration to facilitate complex geometry applications.

- Modeled flow around GTS.

\section{Future Direction}

- Continued development of vortex method approach and application to generic shapes and the GTS geometry. 


\section{Introduction}

During FY03, Caltech continued to improve and extend its vortex method approach to flow past complex bodies. These activities included treatment of spinning boundaries, research on boundary elements, high order isotropic redistribution, development of a fast algorithm for the Closest Point Transform and adaptive time integration. With our colleagues at UCL in Belgium, we also completed an application of our method to the GTS geometry.

\section{Spinning Boundaries}

In our framework, because the fields are computed using the unbounded BiotSavart law, it is necessary to include the contribution of the vorticity present inside any spinning body. The subsequent volume integral is transformed into a surface one, better suited for our boundary element method. Fig. 1G shows the sheet diffused by an impulsively started spinning body; because we included the contribution of the fore mentioned spin vorticity, the sheet - found by a panel solver - has the correct total circulation.

\section{Near-Wall Elements}

Near-wall vorticity can now be represented by attached elements. These panels interact viscously with the regular-free-elements and induce a velocity field (Biot-Savart law). Derivation of an appropriate Biot-Savart law is in progress.

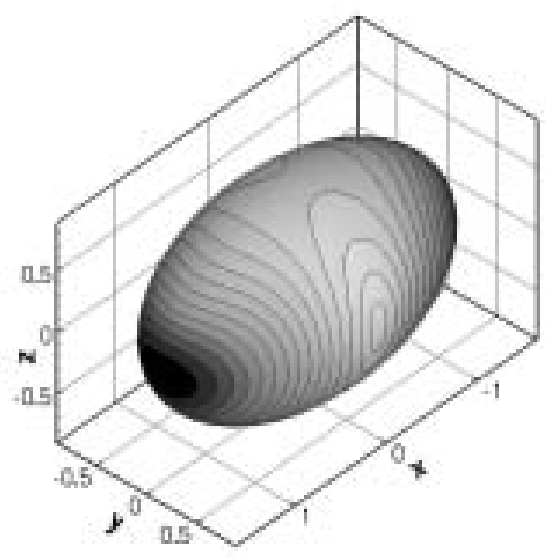

Figure 1G: Vorticity flux magnitude for an impulsively started spinning ellipsoid (rotation about $\mathbf{y}$-axis) .

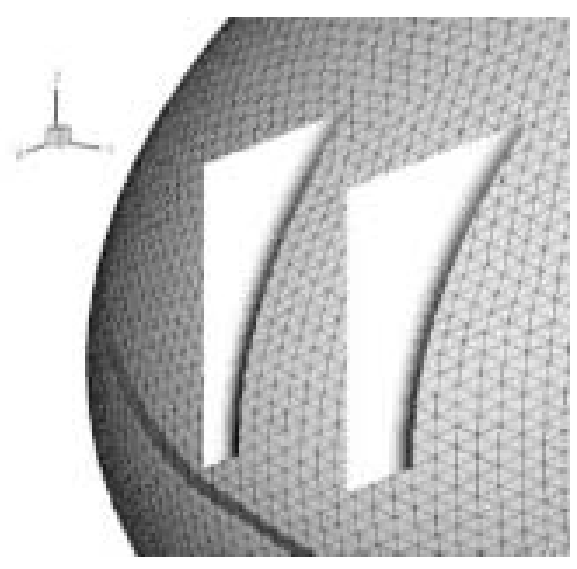

Figure 2G: Near-wall vorticity magnitude at $t$ $=0^{+}$, slices taken near the tip of the ellipsoid.

\section{High Order Isotropic Redistribution}

We pursued the work on the redistribution schemes in Face Centered Cubic (FCC) lattices. A higher order scheme was designed which shows remarkable isotropy when compared to equivalent schemes for cubic lattices (Figs. 3G and 4G). Let us emphasize that the redistribution of the vortex particles every few time steps is critical for the convergence of the method, hence the importance of its isotropy and accuracy. 


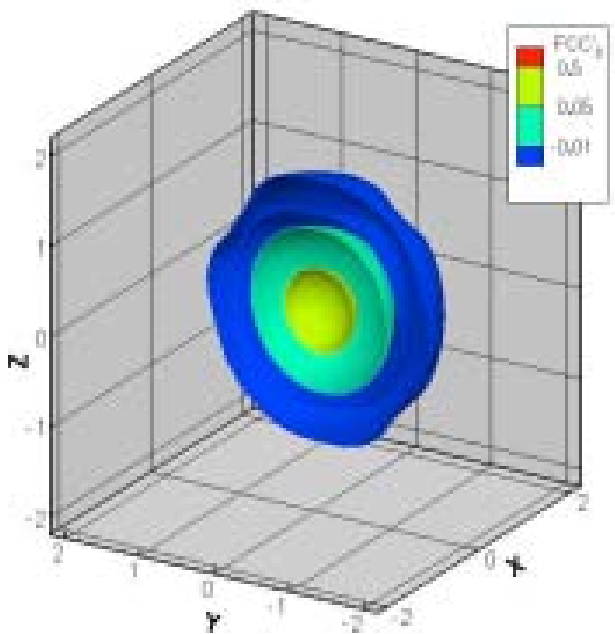

Figure 3G: Isosurfaces of the third order FCC scheme (FCC'3).

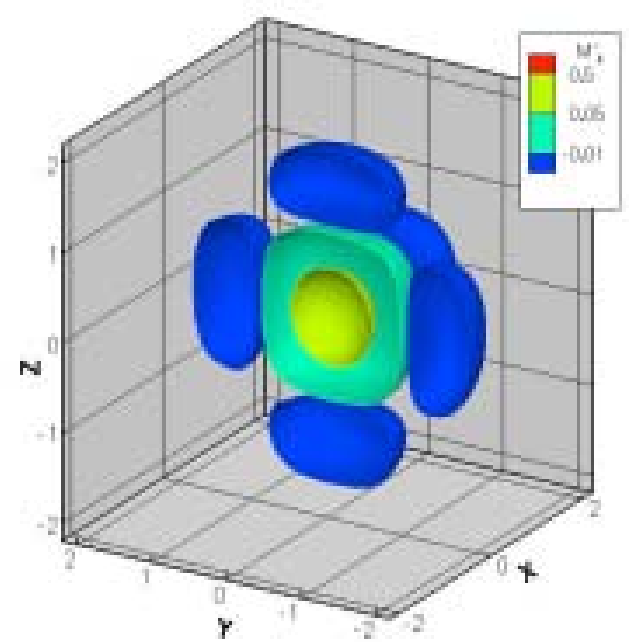

Figure 4: Isosurfaces of the third order cubic scheme (M'4).

\section{Fast Closest Point Transform}

A paper on the new Characteristic / Oct Tree (C/OT) algorithm for the closest point transform, which allows the code to work on arbitrarily complicated boundary geometries, was submitted. The algorithm works by separating space into families of characteristic lines of an Eikonal equation, and sorting them according to how they intersect cells of an oct tree (Figs. 5G and 6G).

This algorithm is now implemented and used in many areas of the code: generation/ redistribution of particles around arbitrary geometries, sampling fields as in Fig. 2G, etc.

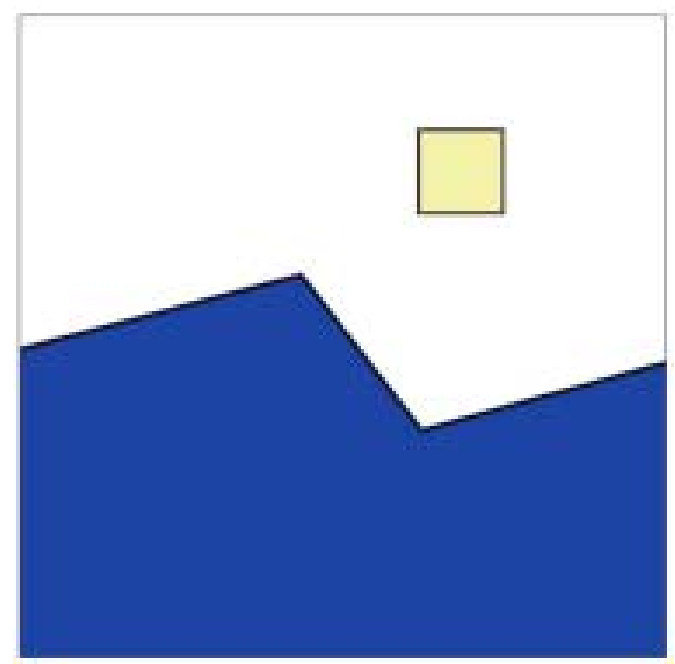

Figure 5G: Boundary and cell of interest.

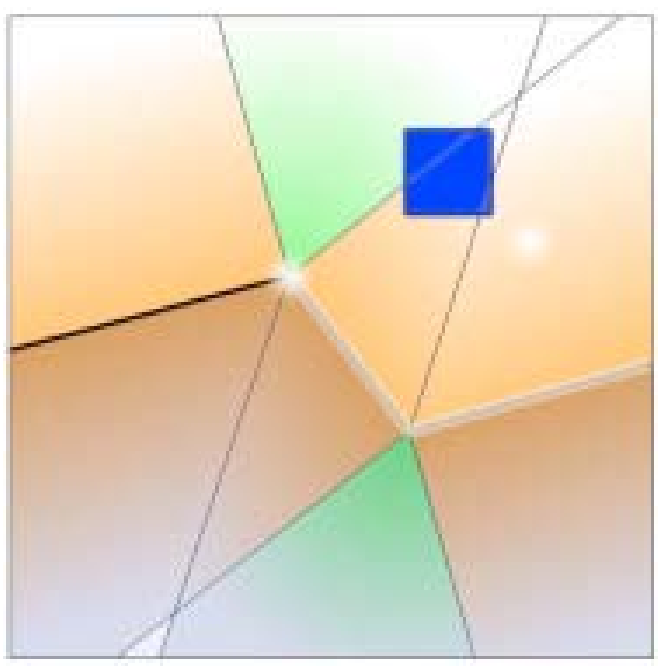

Figure 6: Characteristic regions and intersection with cell

\section{Adaptive Time Integration}

One of the group's ongoing research projects has been the development of an efficient asynchronous dead reckoning 
numerical time integrator for the large systems of differential equations that arise when vortex methods are applied to complicated geometries at high Re. In the past year, work in this area focused on tweaking the strategy to make it scale better to the dense operators typically seen in vortex problems, primarily by eliminating the wake-on-change strategy employed in earlier incarnations of the algorithm.

\section{GTS Model}

The flow around the GTS model was computed in collaboration with $\mathrm{G}$. Winckelmans' group at the Universit'e Catholique de Louvain (UCL). To make this run affordable, attached vortex elements were used on the front part of the truck and free elements were introduced upstream of the region of separation.

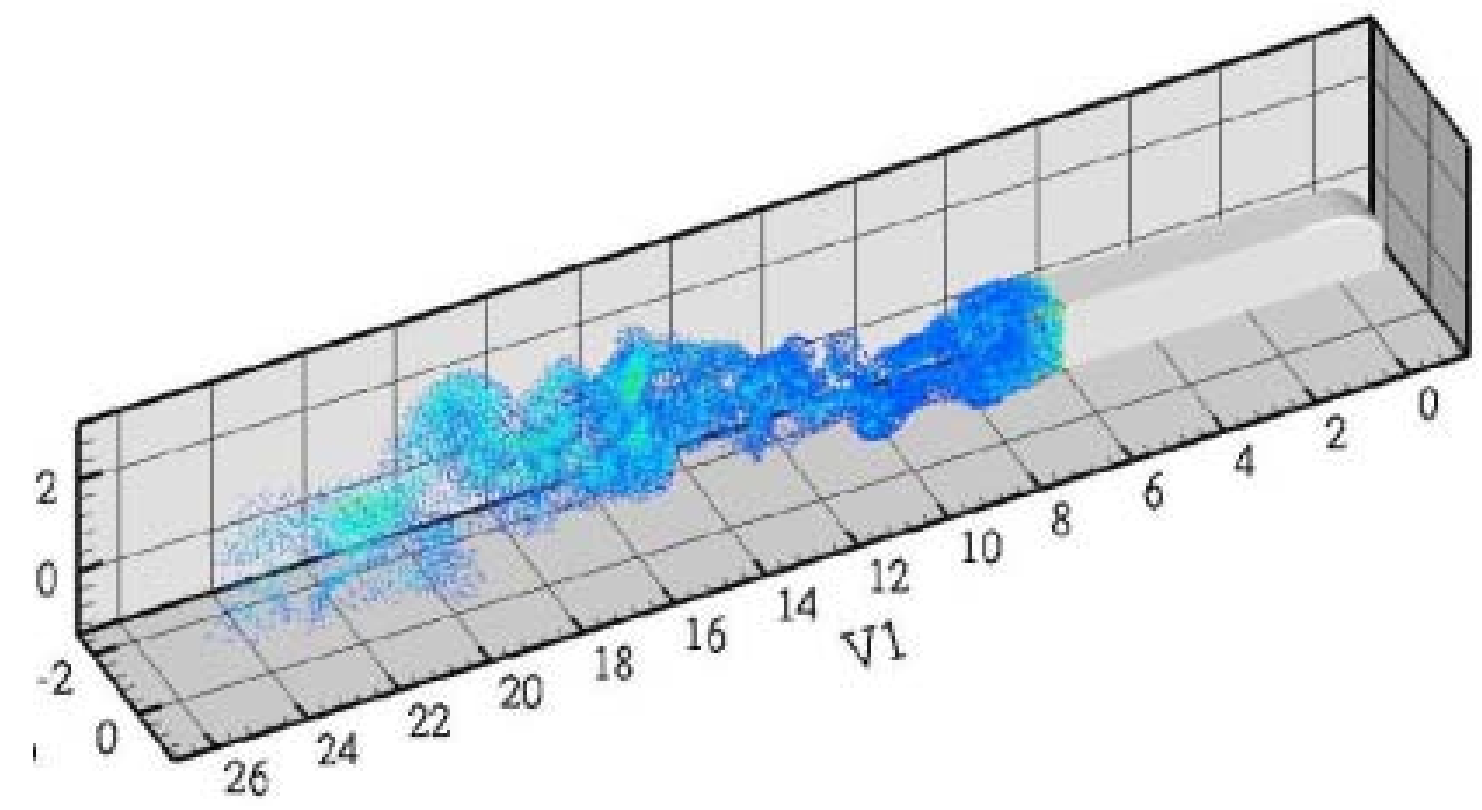

Figure 7: GTS model, positions and strengths of vortex elements. 